TRANSACTIONS OF THE

AMERICAN MATHEMATICAL SOCIETY

Volume 352, Number 12, Pages 5435-5483

S 0002-9947(00)02633-7

Article electronically published on April 13, 2000

\title{
WEAKLY O-MINIMAL STRUCTURES AND REAL CLOSED FIELDS
}

\author{
DUGALD MACPHERSON, DAVID MARKER, AND CHARLES STEINHORN
}

\begin{abstract}
A linearly ordered structure is weakly o-minimal if all of its definable sets in one variable are the union of finitely many convex sets in the structure. Weakly o-minimal structures were introduced by Dickmann, and they arise in several contexts. We here prove several fundamental results about weakly o-minimal structures. Foremost among these, we show that every weakly o-minimal ordered field is real closed. We also develop a substantial theory of definable sets in weakly o-minimal structures, patterned, as much as possible, after that for o-minimal structures.
\end{abstract}

\section{INTRODUCTION}

A linearly ordered structure $\mathfrak{M}=(M,<, \ldots)$ is o-minimal if every definable 11 subset of $M$ is the union of finitely many points and open intervals in $(M,<)$ with endpoints in $M \cup\{-\infty, \infty\}$. O-minimality has been the subject of extensive research over the last fifteen years and is the subject of the monograph [9]. This paper concerns weakly o-minimal structures $\mathfrak{M}=(M,<, \ldots)$, where the hypothesis of o-minimality is relaxed to permit definable subsets of $M$ to be the union of finitely many convex sets in $(M,<)$. We also say that a complete theory $T$ is weakly ominimal if all its models are weakly o-minimal. Weak o-minimality was introduced by Dickmann in [6], where some algebraic examples are developed and some basic facts about weakly o-minimal ordered groups and rings are obtained.

The principal result proved in this paper, Theorem 5.3, establishes that every weakly o-minimal ordered field is real closed. En route to proving this theorem, and for independent interest, we develop a substantial theory of definable sets in weakly o-minimal structures. Throughout the paper we assume that all structures are densely ordered; we make this supposition for convenience and because the primary examples - to our mind - are densely ordered.

We now survey the contents of this paper, beginning with an overview of some of the important examples. Throughout the paper we assume familiarity with basic results of o-minimality as contained [22] and [14] (see also [9]).

Received by the editors April 24, 1998.

2000 Mathematics Subject Classification. Primary 03C60, 03C64.

The second author's research was partially supported by NSF grant DMS-9626856, and the third author's was partially supported by NSF grants DMS-9401723 and DMS-9704869, and SERC grant GR/H57097.

${ }^{1}$ Throughout this paper 'definable' means 'definable with parameters'. 
1.1. Examples. We present several examples of weakly o-minimal structures in Section 2. Some of these have been chosen for their mathematical significance whereas others serve to illustrate various model theoretic phenomena.

Observe first that any weakly o-minimal expansion of $(\mathbb{R},<)$ is o-minimal, as the ordering is Dedekind complete. So these are not a source of interesting examples of weakly o-minimal structures. Non-archimedean real closed fields together with a unary predicate for a non-trivial convex valuation ring provide a familiar class of examples. These structures are bi-interpretable with their valuation rings, known as real closed rings, which were studied from a model-theoretic viewpoint by Cherlin and Dickmann [5]. They prove a quantifier-elimination theorem in [5] see Section 2.1 below for details - from which it follows that real closed rings and convexly valued real closed fields are weakly o-minimal, and moreover that they have weakly o-minimal theories. These results were extended substantially by van den Dries and Lewenberg in [10] and 8, where it is proved that the structures considered by Cherlin and Dickman have proper weakly o-minimal analytic expansions - see Section 2.2 for details.

Another natural example of a weakly o-minimal structure is the ordered field of real algebraic numbers augmented by a unary predicate picking out the convex set $(-\alpha, \alpha)$, where $\alpha$ is any transcendental real number. This situation is treated in Section 2.3 We remark that weak o-minimality of this structure as well as those studied by van den Dries and Lewenberg follows from a surprising recent result of Baisalov and Poizat [3]. Answering a question posed by G. Cherlin and stated in an earlier draft of this paper, they prove that any expansion of an ominimal structure by predicates for convex subsets is weakly o-minimal. Another class of algebraically natural examples are the centipetal contraction groups studied by Kuhlmann in [15] and [16. These expansions of ordered groups, which arise in the study of exponential fields, are discussed in Section 2.4

The model theory of weakly o-minimal structures does not develop quite as smoothly as that of o-minimal structures. We exhibit one of the most striking differences in Example 2.5. where we show that there is a weakly o-minimal structure with an elementary extension that is not weakly o-minimal. Hence a weakly o-minimal structure need not have a weakly o-minimal theory. Example 2.6.1 provides us with a structure whose theory is weakly o-minimal and which has a definable (unary) function that is locally constant and everywhere continuous but not piecewise constant. This shows that the Monotonicity Theorem of [22] fails. It also implies (see Theorem 7.2) that the algebraic closure operator does not in general have the Exchange Property, which hinders the development of a good dimension theory (centripetal contraction groups also provide examples of this). In Examples 2.6.4 and 2.6.5 we see that a weakly o-minimal theory need not have a prime model, again in contrast to what is true in the o-minimal context.

1.2. Sorts in $\bar{M}$. Before discussing the principal results in this paper, we introduce the concept of a sort in $\bar{M}$. We first indicate how this notion is forced upon us in the study of weakly o-minimal structures. Suppose that we wish to prove a cell decomposition theorem for models of a weakly o-minimal theory. Let Th $\mathfrak{M}$ be weakly o-minimal and let $S \subset M^{2}$ be definable. We want to express $S$ as a finite union of cells, under some reasonable definition of 'cell.' Let $\pi: M^{2} \longrightarrow M$ denote projection onto the first coordinate, and let $I:=\pi(S)$, a finite union of convex subsets of $M$. By weak o-minimality, for each $a \in M$ the fiber $S_{a}:=\{y:(a, y) \in S\}$ 
is the union of finitely many convex sets, some of them possibly singletons. For simplicity we assume that $I$ is convex, and that each $S_{a}$ is an infinite convex set. If each $S_{a}$ is an interval, that is, has endpoints in $M \cup\{-\infty, \infty\}$, then there are definable functions $f, g: M \longrightarrow M \cup\{-\infty, \infty\}$ where $f(x):=\inf S_{x}, g(x)=\sup S_{x}$, and in the notation of [14] we have $S=(f, g)_{I}$. In the o-minimal case, by cutting down $I$ using the Monotonicity Theorem we can assume also that $f$ and $g$ are continuous and hence that $S$ is a cell. Difficulties arise in the weakly o-minimal case if the sets $S_{a}$ are convex but do not have endpoints in $M$. We can handle this complication by regarding $f$ and $g$ as definable functions from $M$ to its Dedekind completion, $\bar{M}$, and it is for exactly this situation that we introduce sorts as follows.

Let $Y \subset M^{n+1}$ be 0-definable, let $\pi: M^{n+1} \longrightarrow M^{n}$ be the projection which drops the last coordinate, and let $Z:=\pi(Y)$. For each $\bar{a} \in Z$ let

$$
Y_{\bar{a}}:=\{y:(\bar{a}, y) \in Y\} \text {. }
$$

Suppose that for every $\bar{a} \in Z$ the set $Y_{\bar{a}}$ is bounded above but does not have a supremum in $M$. We let $\sim$ be the 0 -definable equivalence relation on $M^{n}$ given by

$$
\begin{gathered}
\bar{a} \sim \bar{b} \text { for all } \bar{a}, \bar{b} \in M^{n} \backslash Z, \\
\bar{a} \sim \bar{b} \Leftrightarrow \sup Y_{\bar{a}}=\sup Y_{\bar{b}} \text { if } \bar{a}, \bar{b} \in Z .
\end{gathered}
$$

Let $\bar{Z}:=Z / \sim$, and for each $\bar{a} \in Z$ we denote by $[\bar{a}]$ the $\sim$-class of $\bar{a}$. There is a natural 0-definable total order on $M \cup \bar{Z}$, defined as follows. Let $\bar{a} \in Z$ and $c \in M$. Then $[\bar{a}]<c$ if and only if $w<c$ for all $w \in Y_{\bar{a}}$. If $\bar{a} \not \bar{b}$ then there is some $x \in M$ such that $[\bar{a}]<x<[\bar{b}]$ or $[\bar{b}]<x<[\bar{a}]$, and so $<$ induces a total order on $M \cup \bar{Z}$. We call such a set $\bar{Z}$ a sort in $\bar{M}$, and view $\bar{Z}$ as naturally embedded in $\bar{M}$. Similarly, we can obtain a sort in $\bar{M}$ by considering infima instead of suprema. It is important to bear in mind that a sort depends on the set $Y$, and not just on the equivalence relation $\sim$ defined on its projection $\pi(Y)$. So if we regard sorts just as subsets of $M^{e q}$, the same subset of $M^{e q}$ may embed in several different ways in $\bar{M}$. In this setting a definable function $F: M^{n} \longrightarrow \bar{M}$ is understood to be a definable function $M^{n} \longrightarrow \bar{Z}$, where $\bar{Z}$ is a sort.

1.3. The main results of the paper. In Section 3 we present the best analogues of the monotonicity theorems of [22] that we were able to prove. These theorems are for definable functions $f: M \longrightarrow \bar{M}$. Because of the examples mentioned above, it is not possible to obtain full piecewise monotonicity in the weakly o-minimal setting. We are able to prove that every such definable function in a structure $\mathfrak{M}$ is piecewise locally monotonic or piecewise locally constant - in which case we say that $\mathfrak{M}$ has monotonicity - under one of two additional hypotheses: that the theory is weakly o-minimal (Theorem 3.3 ) or that the stucture is a weakly o-minimal expansion of an ordered group (Theorem 3.4) 2

In Section 4 we obtain a rather weak form of cell decomposition for models of weakly o-minimal theories (Theorem 4.6). The result is weak because we merely require that the boundary functions of the cells are definable and not that they are continuous. More importantly, we show in Section 4 that there is a reasonable notion of topological dimension for definable sets in a weakly o-minimal structure $\mathfrak{M}$

\footnotetext{
${ }^{2}$ These additional hypotheses are removed in the recent preprint [2] of R. Arefiev-see the beginning of Section 3 for details
} 
provided that $\mathfrak{M}$ has monotonicity 3 For a set $X \subset M^{n}$ definable in $\mathfrak{M}$ the (topological) dimension of $X$ is defined to be the greatest $r$ for which there is a projection $p: M^{n} \longrightarrow M^{r}$ such that $p(X)$ has non-empty interior in $M^{r}$. Then (assuming that $\mathfrak{M}$ has monotonicity), if $X_{1}, \ldots, X_{t}$ are definable subsets of $M^{n}$ and $X=\bigcup_{i=1}^{t} X_{i}$, we obtain in Theorem 4.2 that $\operatorname{dim}(X)=\max \left\{\operatorname{dim}\left(X_{1}\right), \ldots, \operatorname{dim}\left(X_{t}\right)\right\}$. In particular, it is impossible to define a dense, codense subset of a box in $M^{n}$. In case $\mathfrak{M}$ has a weakly o-minimal theory, this notion of dimension is shown also to enjoy other good properties: it is preserved by definable bijections (Theorem 4.7); and, if algebraic closure has the exchange property, then the dimension obtained from algebraic closure coincides with topological dimension (Theorem 4.12). In the process of proving these results, we obtain a slightly improved cell decomposition in Theorem 4.11. However, still, the cells involved do not necessarily have continuous boundary functions.

In Section 5 we turn to the proof of the main result of this paper, Theorem 5.3 that every weakly o-minimal ordered field is real closed. We sketch here the main ideas behind the proof. Let $F$ be a weakly o-minimal ordered field. Applying the results of Sections 3 and 4 we first prove a version of the inverse function theorem (Theorem [5.7) for polynomial functions $F^{n} \longrightarrow F^{n}$ (Proposition 5.9). This, together with adaptations of arguments in [11, enables us to show that the weakly o-minimal ordered field $F$ is topologically closed in its real closure (Proposition 5.4). The theorem now follows immediately if $F$ is archimedean. So suppose it is not. We now consider the corresponding archimedean valuation $v$ with residue field $\bar{F}$. It is immediate that the value group is divisible, and a short argument-using Proposition 5.4 yields that $\bar{F}$ is real closed. Thus, by a result of MacLane [18] (see also [23]) it suffices to show that $(F, v)$ is henselian. Another short argument establishes this, using a coarsening of the valuation, and Theorem 5.3 is proved. We also establish in Section 5 that every weakly o-minimal ordered group is divisible abelian.

In Section 6 we study weakly o-minimal expansions of ordered fields according to whether or not there exists a non-trivial definable convex valuation. Theorem 6.3 characterizes when a weakly o-minimal expansion of an ordered field $\mathfrak{M}$ has such a valuation in terms of the definable cuts that exist in $\mathfrak{M}$. Most of the section is given over to proving that if there is no definable convex valuation in $\mathfrak{M}$ in which case we say that $\mathfrak{M}$ is of non-valuational type-then much of the good o-minimal structure theory goes through. Specifically: every model of Th $\mathfrak{M}$ is weakly o-minimal; definable functions are piecewise continuous and constant or strictly monotonic; and, cell decomposition with continuous boundary functions holds. The real algebraic numbers, expanded by a predicate for the convex set $(-\alpha, \alpha)$, where $\alpha$ is any transcendental real number, provide the most obvious examples of weakly o-minimal expansions of ordered fields of non-valuational type.

The final section of paper, Section 7 collects several miscellaneous observations and includes some open questions. Theorem 7.2 shows that, for a weakly o-minimal structure, several conditions are equivalent to the condition that algebraic closure does not have the exchange property. We also prove that a weakly o-minimal theory does not have the independence property, generalizing a result of [22]. This gives an alternative proof of the result of Mathews [20] that real closed rings do not have the independence property. We pose some open questions at the end of Section 7 .

\footnotetext{
${ }^{3}$ Note that Arefiev's result [2] shows that the additional hypothesis is not needed.
} 
1.4. Notations and Conventions. Most of the terminology used in the paper is standard. We use $\mathcal{M}, \mathcal{N}, \ldots, \mathfrak{M}, \mathfrak{N}, \ldots$ to denote structures, which, unless otherwise stated, are assumed to be expansions of dense linear orders. Upper case roman letters denote subsets of the universe of a structure, and lower case roman letters are used to designate elements of a structure.

Let $\mathcal{M}=(M,<, \ldots)$ be an ordered structure. Making explicit the usage we have tacitly adopted throughout the introduction, a subset $I \subseteq M$ is said to be convex (in $\mathcal{M}$ ) if whenever $a, b \in I$ and $a<x<b$ we have $x \in I$. Also, an interval in $\mathcal{M}$ is a convex set having both a supremum and infimum in $M \cup\{ \pm \infty\}$. As we have done above, we use $\bar{M}$ to denote the Dedekind completion of $(M,<)$. For a structure $\mathcal{M}$ and $A, B \subseteq M$ we write $A<B$ if $a<b$ for all $a \in A$ and $b \in B$. If $A \subset M$ and $x \in M$, we write $A<x$ instead of $A<\{x\}$. For subsets of $M^{n}$, all topological notions used in this paper refer to the product topology induced by the order topology on $(M,<)$. By a box $B \subseteq M^{n}$ we always mean a box whose sides are parallel to the coordinate axes. For $S \subseteq M^{n}$, we use $\operatorname{Int}(S)$ for the interior of $S$ in $M^{n}$.

1.5. Acknowledgment. During the time that an earlier version of this paper circulated in preprint form we have received many useful comments and corrections from Bektur Baizhanov and his colleagues in Almaty, Kazakhstan. We acknowledge with gratitude their interest in this work, and note that this version of the paper is much improved over its predecessor as a result.

\section{EXAMPLES OF WEAKLY O-MiNimal STRUCTURES}

Here we offer several examples of weakly o-minimal structures and theories. The first four illustrate the range of contexts in which weakly o-minimal structures appear, and the remaining examples are provided to demonstrate several model theoretic phenomena that distinguish weak o-minimality from o-minimality. We note again that weak o-minimality of several of the structures presented here can be obtained by the result of Baisalov and Poizat [3] mentioned in 1.1 .

2.1. Real closed rings. Cherlin and Dickmann in [5] define a real closed ring $\mathcal{R}=(R,+, \cdot, 0,1,<)$ to be a commutative ordered domain with unit such that every polynomial in a single variable with coefficients in $R$ has the intermediate value property. In this same paper they examine real closed rings from a model theoretic point of view. Let $\mathcal{R}$ be a commutative ordered domain with unit. For $x, y \in R$, we write $x$ Div $y$ if $\mathcal{R} \models \exists z x z=y$. The ring $\mathcal{R}$ is said to be a convexly ordered valuation ring if it is not a field and

$$
\mathcal{R}=\forall x \forall y(|x| \leq|y| \Longrightarrow y \operatorname{Div} x) .
$$

This condition is equivalent to several others, such as that $R$ is a convex subring of its field of fractions (see [5], for example). Cherlin and Dickmann [5] characterize real closed rings as exactly the convex subrings (containing 1 ) of real closed fields. They further prove that real closed rings have quantifier elimination in the language $L_{\text {Div }}$ of ordered rings with the Div predicate. As pointed out in the introduction to [10], real closed rings and the quantifier elimination just described have been applied in the solution of problems in real algebraic geometry.

Dickmann in [6] observes that real closed rings are weakly o-minimal. $\mathrm{He}$ also proves that every convexly ordered valuation ring which admits quantifierelimination in the language $L_{\text {Div }}$ is a real closed ring. One of the main results of 
this paper, Theorem 5.3 and its consequence, Corollary [5.13, establishes that every weakly o-minimal ordered commutative ring with unit is real closed. We remark that these results imply that if an ordered ring with unit is weakly o-minimal, then so is every elementarily equivalent ordered ring. This is not generally true - see 2.5 below.

2.2. Expansions of real closed valued fields. For a pure real closed valued field $\mathcal{R}$ - that is, in the language of ordered rings augmented by a predicate for a convex valuation ring - it is shown in [5] that the residue field $\bar{R}$ is real closed and the value group $\Gamma$ is divisible. In the case of real closed valued fields, it was known that $\bar{R}$, respectively $\Gamma$, inherits no structure from $\mathcal{R}$ beyond that of a real closed field, respectively, divisible ordered abelian group (see [8] for a discussion of what was known and references).

These results are extended substantially in [10] and [8] as follows. Let $\mathcal{R}=$ $(R, \ldots)$ be an o-minimal expansion of a real closed field and let $V \subseteq R$ be a proper $T$-convex subring of $\mathcal{R}$, that is, a proper convex subring of $R$ closed under application of continuous 0-definable functions. Then the theory of the pair $(\mathcal{R}, V)$ is weakly o-minimal. Moreover, the residue field $\bar{R}$ of $\mathcal{R}$ can be made canonically into a model of $T=\operatorname{Th}(\mathcal{R})$ (see [10]). In 8 it is proved that no additional structure is induced on $\bar{R}$ by $(\mathcal{R}, V)$, and in particular that $\bar{R}$ together with all the a priori induced structure is o-minimal. For $\Gamma$ the situation is different. If $T$ is power bounded (see [8] for the definition 4 ) then $\Gamma$ together with all the structure induced on it by $(\mathcal{R}, V)$ is o-minimal. If, however, $T$ is not power bounded, then the theory of $\Gamma$ with the additional induced structure is only weakly o-minimal (see [10], Section 4).

2.3. Real algebraic numbers expanded by a convex set. We present here a weakly o-minimal expansion of an ordered field fundamentally different from the preceding examples - this difference is the starting point for Section 6 . We prove the following proposition. Let $\mathcal{R}_{\text {alg }}$ denote the ordered field of real algebraic numbers, $\left(R_{\text {alg }},+, \cdot, 0,1,<\right)$, and let $L=\{+, \cdot, 0,1,<\}$ be the language of ordered fields.

Proposition 2.1. Let $\alpha$ be a real transcendental number and let the unary predicate symbol $P$ be interpreted by the convex set $S:=(-\alpha, \alpha) \cap R_{\text {alg }}$. Then $\mathfrak{R}:=\left(\mathcal{R}_{\text {alg }}, S\right)$ is weakly o-minimal.

The proof we give actually shows that Th $\mathfrak{R}$ is weakly o-minimal. Also, it is easily adjusted to show that if $\left\{\alpha_{i}: i \in I\right\}$ is any algebraically independent subset of $\mathbb{R}$ and $S_{i}:=\left(-\alpha_{i}, \alpha_{i}\right)$ for each $i \in I$, then $\left(\mathcal{R}_{\text {alg }}, S_{i}\right)_{i \in I}$ has a weakly o-minimal theory. We also note that the weak o-minimality of $\mathfrak{R}$ is a direct consequence of [3]; we present the proof below because it is constructive and may be of independent interest.

Proof. We expand the language of $\mathfrak{R}$ by adjoining, for each $m>0$, the definable relation $R_{m}\left(v_{0}, \ldots, v_{m}\right)$, which asserts that

$$
\begin{aligned}
\exists z_{1}, z_{2}\left[P\left(z_{1}\right)\right. & \wedge \forall w\left(P(w) \rightarrow z_{2}>w\right) \\
& \left.\wedge \forall u\left(\left(z_{1}<u<z_{2}\right) \rightarrow\left(v_{0}+v_{1} u+\ldots+v_{m} u^{m}>0\right)\right)\right] .
\end{aligned}
$$

\footnotetext{
${ }^{4}$ For the purposes of this discussion the reader may take power bounded to be polynomially bounded, i.e., every definable function in $\mathcal{R}$ is majorized at infinity by a polynomial function.
} 
We denote by $L^{*}$ the expansion of $L$ which includes $P$ and these relations, and let $\mathfrak{R}^{*}$ be the expansion of $\mathfrak{R}$ to $L^{*}$. Let $R_{\text {alg }}\langle\alpha\rangle$ denote the real closure of $R_{\text {alg }} \cup\{\alpha\}$ and let $\mathcal{R}_{\text {alg }}\langle\alpha\rangle:=\left(R_{\text {alg }}\langle\alpha\rangle,+, \cdot, 0,1,<\right)$.

Claim 2.1.1. Let $\phi\left(x_{1}, \ldots, x_{n}\right)$ be a quantifier-free $L^{*}$-formula. Then there is an $L$-formula $\psi_{\phi}\left(x_{1}, \ldots, x_{n}, y\right)$ so that for all $a_{1}, \ldots, a_{n} \in R_{\text {alg }}$ we have

$$
\mathfrak{R}^{*} \models \phi\left(a_{1}, \ldots, a_{n}\right) \Longleftrightarrow \mathcal{R}_{\text {alg }}\langle\alpha\rangle \models \psi_{\phi}\left(a_{1}, \ldots, a_{n}, \alpha\right) .
$$

Proof. We need only produce formulas $\psi_{\phi}$ corresponding to the atomic formulas $P$ and $R_{m}$ for $m>0$. If $\phi(x)$ is $P(x)$, let $\psi_{P}(x, \alpha)$ be $-\alpha<x<\alpha$, and if $\phi$ is $R_{m}\left(x_{0}, \ldots, x_{m}\right)$, let $\psi_{R_{m}}\left(x_{0}, \ldots, x_{m}, \alpha\right)$ be

$$
x_{0}+x_{1} \alpha+\ldots+x_{m} \alpha^{m}>0 .
$$

Claim 2.1.2. Let $X \subseteq\left(R_{\text {alg }}\langle\alpha\rangle\right)^{n}$ be L-definable with parameters in $R_{\text {alg }} \cup\{\alpha\}$. Then $X \cap\left(R_{\text {alg }}\right)^{n}$ is quantifier-free definable in $\mathfrak{R}^{*}$.

Proof. As the set $X$ is semialgebraic, we have just two cases to consider. Let $p\left(x_{1}, \ldots, x_{n}, \alpha\right) \in R_{\text {alg }}\left[x_{1}, \ldots, x_{n}, \alpha\right]$, with $p(\bar{x})=\sum_{i=0}^{k} q_{i}(\bar{x}) \alpha^{i}$. First, for all $\bar{a}=\left(a_{1}, \ldots, a_{n}\right) \in\left(R_{\text {alg }}\right)^{n}$, we have

$$
\begin{aligned}
\mathcal{R}_{\text {alg }}\langle\alpha\rangle \models p\left(a_{1}, \ldots, a_{n}, \alpha\right)>0 & \\
& \Longleftrightarrow \mathcal{R}_{\text {alg }}\langle\alpha\rangle \models \exists z_{1}, z_{2}\left(z_{1}<\alpha<z_{2} \wedge \forall w \in\left(z_{1}, z_{2}\right) p(\bar{a}, w)>0\right) \\
& \Longleftrightarrow \mathfrak{R}^{*} \models R_{k}\left(q_{0}(\bar{a}), \ldots, q_{k}(\bar{a})\right) .
\end{aligned}
$$

Second, as $\alpha$ is transcendental over $R_{\text {alg }}$ we see that

$$
\mathcal{R}_{\mathrm{alg}}\langle\alpha\rangle \models p(\bar{a}, \alpha)=0 \Longleftrightarrow \mathfrak{R}^{*} \models \bigwedge_{i=0}^{k} q_{i}(\bar{a})=0 .
$$

We next assert that

Claim 2.1.3. The structure $\mathfrak{R}^{*}$ admits elimination of quantifiers.

Proof. Suppose that $\exists y \phi(\bar{x}, y)$ is an $L^{*}$-formula, where $\phi(\bar{x}, y)$ is quantifier-free and $\bar{x}=\left(x_{1}, \ldots, x_{n}\right)$. By Claim 2.1.1 there is an $L$-formula $\psi_{\phi}(\bar{x}, y, w)$ so that for all $\bar{a}, b \in R_{\text {alg }}$ we have

$$
\mathfrak{R}^{*}=\phi(\bar{a}, b) \Longleftrightarrow \mathcal{R}_{\text {alg }}\langle\alpha\rangle \models \psi_{\phi}(\bar{a}, b, \alpha) .
$$

We now use the o-minimality of $\mathcal{R}_{\text {alg }}\langle\alpha\rangle$ to write

$$
B:=\left\{(\bar{a}, b) \in\left(R_{\text {alg }}\langle\alpha\rangle\right)^{n+1}: \mathcal{R}_{\text {alg }}\langle\alpha\rangle \models \psi_{\phi}(\bar{a}, b, \alpha)\right\}
$$

as a disjoint union of cells $C_{1}, \ldots, C_{k}$, in the usual sense of cells for o-minimal structures. For all $\bar{a} \in\left(R_{\text {alg }}\right)^{n}$ we clearly have

$$
\begin{aligned}
\mathfrak{R}^{*} \models \exists y \phi(\bar{a}, y) & \Longleftrightarrow \quad\left(\exists y \in R_{\mathrm{alg}}\right) \bigvee_{i=1}^{k}(\bar{a}, y) \in C_{i} \\
& \Longleftrightarrow \bigvee_{i=1}^{k}\left(\exists y \in R_{\mathrm{alg}}\right)(\bar{a}, y) \in C_{i} .
\end{aligned}
$$

Let $\pi:\left(R_{\text {alg }}\langle\alpha\rangle\right)^{n+1} \longrightarrow\left(R_{\text {alg }}\langle\alpha\rangle\right)^{n}$ denote projection onto the first $n$ coordinates. We have two cases, based on the usual recursive definition of cells in o-minimal structures (as in [14]). Let $i \in\{1, \ldots, k\}$. 
Case 1. $C_{i}=(f, g)_{\pi\left(C_{i}\right)}=\left\{(\bar{a}, b) \in\left(R_{\mathrm{alg}}\langle\alpha\rangle\right)^{n+1}: \bar{a} \in \pi\left(C_{i}\right) \wedge f(\bar{a})<b<g(\bar{a})\right\}$, where $f$ and $g$ are definable (in $\mathcal{R}_{\mathrm{alg}}\langle\alpha\rangle$ ) and continuous on $\pi\left(C_{i}\right)$, and $f(\bar{x})<g(\bar{x})$ for all $\bar{x} \in \pi\left(C_{i}\right)$.

In this case, since $R_{\text {alg }}$ is dense in $R_{\text {alg }}\langle\alpha\rangle$, we have for all $\bar{a} \in\left(R_{\text {alg }}\right)^{n}$ that

$$
\left(\exists y \in R_{\text {alg }}\right)(\bar{a}, y) \in C_{i} \Longleftrightarrow \bar{a} \in \pi\left(C_{i}\right) .
$$

To complete the proof of the claim in this case, note by Claim 2.1.2 that $\pi\left(C_{i}\right) \cap$ $\left(R_{\text {alg }}\right)^{n}$ is quantifier-free definable in $\mathfrak{R}^{*}$.

Case 2. $C_{i}$ is the graph of a function $f \uparrow_{\pi\left(C_{i}\right)}$, where $f$ is definable $\left(\right.$ in $\left.\mathcal{R}_{\text {alg }}\langle\alpha\rangle\right)$ and continuous on $\pi\left(C_{i}\right)$.

In this case, by quantifier-elimination for the $L$-structure $\mathcal{R}_{\text {alg }}\langle\alpha\rangle$, we may assume that there is a polynomial $p(\bar{x}, y, \alpha)=\sum_{i=0}^{k} q_{i}(\bar{x}, y) \alpha^{i}$, where $q_{i}(\bar{x}, y) \in$ $R_{\text {alg }}[\bar{x}, y]$ for each $i$, such that for each $\bar{a} \in \pi\left(C_{i}\right)$ the polynomial $p(\bar{a}, y, \alpha)$ has only finitely many zeros and $p(\bar{a}, f(\bar{a}), \alpha)=0$. In this case, as $\alpha$ is transcendental, for $\bar{a}, b \in R_{\text {alg }}$, we have

$$
p(\bar{a}, b, \alpha)=0 \Longleftrightarrow \bigwedge_{i=0}^{k} q_{i}(\bar{a}, b)=0 .
$$

Then, for $\bar{a}, b \in R_{\text {alg }}$ with $\bar{a} \in \pi\left(C_{i}\right) \cap\left(R_{\text {alg }}\right)^{n}$, we may assume that there is some $m \in \mathbb{N}$ such that

$$
\mathcal{R}_{\text {alg }}\langle\alpha\rangle \models b=f(\bar{a}) \Longleftrightarrow \mathcal{R}_{\text {alg }}\langle\alpha\rangle \models \text { ' } b \text { is the } m^{\text {th }} \text { root of } \sum_{i=0}^{k}\left(q_{i}(\bar{a}, y)\right)^{2}=0 \text { '. }
$$

Let $\chi(\bar{x}, z)$ be the $L$-formula ' $z$ is the $m^{\text {th }}$ root of $\sum_{i=0}^{k}\left(q_{i}(\bar{x}, y)\right)^{2}=0$ ' (with parameters in $\mathcal{R}_{\text {alg }}$ ). By quantifier elimination for real closed fields we may take $\chi$ to be quantifier-free, and so, as $\mathfrak{R}^{*}$ is an expansion of $\mathcal{R}_{\text {alg }}$, we have for all $\bar{a}, b \in R_{\text {alg }}$ that

$$
\mathcal{R}_{\text {alg }}\langle\alpha\rangle \models \chi(\bar{a}, b) \Longleftrightarrow \mathfrak{R}^{*} \models \chi(\bar{a}, b) .
$$

Hence, for $\bar{a} \in\left(R_{\mathrm{alg}}\right)^{n}$, we have

$$
\left(\exists z \in R_{\text {alg }}\right)(\bar{a}, z) \in C_{i} \Longleftrightarrow \bar{a} \in \pi\left(C_{i}\right) \text { and } \mathcal{R}_{\text {alg }} \models \exists z \chi(\bar{a}, z) .
$$

To finish the argument in this case, and with it the proof of the claim, we apply Claim 2.1.2 and quantifier elimination for real closed fields to see that the right hand side is quantifier-free definable in $\mathfrak{R}^{*}$.

To complete the proof of the proposition it suffices to check that every $\mathfrak{R}^{*}$ definable subset of $R_{\mathrm{alg}}$ is the union of finitely many convex sets. For this, by Claim [2.1.3] it is enough to show that any quantifier-free $\mathfrak{R}^{*}$-definable subset is of this form. This follows easily by Claim 2.1.1 and the o-minimality of $\mathcal{R}_{\text {alg }}\langle\alpha\rangle$.

2.4. Contraction groups. We here mention recent work of F-V. Kuhlmann [15. Let $(G,<,+)$ be a divisible ordered abelian group, and let $\chi: G \longrightarrow G$ be a function satisfying the following axioms:

(i) $(\forall x) \chi(x)=0 \leftrightarrow x=0$,

(ii) $\chi$ preserves $\leq$,

(iii) $\forall x(\chi(-x)=-\chi(x))$,

(iv) if $x, y$ are archimedean equivalent, and both positive or both negative, then $\chi(x)=\chi(y)$,

(v) $\chi$ is surjective, 
(vi) $\forall x(|x|>|\chi(x)|)$.

In [15] the resulting structure $\mathcal{G}=(G,<,+, 0, \chi)$ is called a (divisible) centripetal contraction group. It is shown in [15] and [16] that $\mathcal{G}$ has a weakly o-minimal theory by proving that the axioms of divisible ordered abelian groups together with (i)-(vi) axiomatize a complete theory $T_{\mathrm{cg}}$ with elimination of quantifiers. The study of contraction groups is motivated by their natural examples: value groups of nonarchimedean exponential fields with respect to the archimedean valuation, with the contraction induced by the logarithmic function (see Example 2.2).

Because $\chi$ sets up a correspondence between archimedean classes and singletons and we may work in an $\omega$-saturated model of the theory $T_{\text {cg }}$, it follows from Theorem 7.2 in this paper that for $T_{\text {cg }}$ algebraic closure does not have the exchange property. This was proved first in [16], where it was shown in that the algebraic closure of any set in a model of $T_{\text {cg }}$ is just its divisible hull. Contraction groups provide the only algebraic example known to us of a weakly o-minimal theory without the exchange property.

2.5. A weakly o-minimal structure whose theory is not weakly o-minimal. Let $\mathfrak{M}=(M,<, P)$ be the structure with universe $M=\{1,2, \ldots\} \times \mathbb{Q}$ such that $<$ is the lexicographic order, and the binary relation $P$ is defined in $\mathfrak{M}$ by

$$
P((m, p),(n, q)) \Leftrightarrow m=n \wedge \bigvee_{i=0}^{m} q=p+i .
$$

We first observe that if $\mathfrak{M} \preceq \mathfrak{N}$ and $a \in N$ is such that $a>b$ for all $b \in M$, then $\{y: \mathfrak{N} \models P(a, y)\}$ is infinite and discrete. Thus $\mathfrak{M}$ does not have a weakly o-minimal theory. Now we show that $\mathfrak{M}$ is weakly o-minimal.

Let $A_{n}(v)$ be the formula

$$
\exists y_{0} \cdots \exists y_{n}\left[\bigwedge_{i \neq j} y_{i} \neq y_{j} \wedge \bigwedge_{i \leq n} P\left(v, y_{i}\right) \wedge \forall z\left(P(v, z) \rightarrow \bigvee_{i \leq n} z=y_{i}\right)\right] .
$$

Note that the formula $A_{n}(v)$ defines the set $\{n\} \times \mathbb{Q}$ in $\mathfrak{M}$.

For $\mathfrak{N}=$ Th $\mathfrak{M}$ let $A_{n}^{\mathfrak{N}}$ denote the subset of $N$ defined by $A_{n}(v)$. Suppose for each $n \in \mathbb{N} \backslash\{0\}$ that $\rho_{n}$ is an automorphism of $A_{n}^{\mathfrak{N}}$ with the induced structure. Then the mapping $\rho: N \longrightarrow N$ such that $\rho\left\lceil A_{n}^{\mathfrak{N}}=\rho_{n}\right.$ for all $n$ and $\rho$ is the identity on $N \backslash \bigcup_{n \geq 1} A_{n}^{\mathfrak{N}}$ is an automorphism of $\mathfrak{N}$.

To prove that $\mathfrak{M}$ is weakly o-minimal, we must show that every $X \subset M$ definable in $\mathfrak{M}$ is the union of finitely many convex subsets of $M$. Let $a_{1}, \ldots, a_{k} \in M$, and let $\phi\left(v, w_{1}, \ldots, w_{k}\right)$ be a formula such that $X=\left\{c \in M: \mathfrak{M} \models \phi\left(c, a_{1}, \ldots, a_{k}\right)\right\}$. Choose $n_{0}$ so that $a_{1}, \ldots, a_{k} \in A_{1}^{\mathfrak{M}} \cup \cdots \cup A_{n_{0}}^{\mathfrak{M}}$. By the observation made in the preceding paragraph it follows that $X \cap\left(A_{1}^{\mathfrak{M}} \cup \cdots \cup A_{n_{0}}^{\mathfrak{M}}\right)$ is definable in the substructure $\mathfrak{M}_{0}$ of $\mathfrak{M}$ whose universe is $A_{1}^{\mathfrak{M}} \cup \cdots \cup A_{n_{0}}^{\mathfrak{M}}$. Certainly $\mathfrak{M}_{0}$ is weakly o-minimal - for example it is (obviously) definable as a one-dimensional set in the o-minimal structure $(\mathbb{Q},+,<)$. Also, if $n>n_{0}$, then for all $x, y \in A_{n}^{\mathfrak{M}}$ there is an automorphism of $\mathfrak{M}$ that fixes $a_{1}, \ldots, a_{k}$ and maps $x$ to $y$. It follows that for every $n>n_{0}$ either $A_{n}^{\mathfrak{M}} \subset X$ or $A_{n}^{\mathfrak{M}} \cap X=\emptyset$.

To prove that $\mathfrak{M}$ is weakly o-minimal it thus suffices to show either that $x \in X$ for all sufficiently large $x \in M$, or that $x \notin X$ for all sufficiently large $x \in M$. For a contradiction, suppose not. Let $\mathfrak{M}^{*} \succeq \mathfrak{M}$ be saturated (this is a harmless assumption since we could assume GCH if needed) and let $\eta$ denote the order type 
of a saturated dense linear order without endpoints of cardinality $\left|M^{*}\right|$. There are $a, b \in M^{*} \backslash \bigcup_{n \geq 1} A_{n}^{\mathfrak{M}^{*}}$ such that

$$
\mathfrak{M}^{*} \models \phi\left(a, a_{1}, \ldots, a_{k}\right) \wedge \neg \phi\left(b, a_{1}, \ldots, a_{k}\right) .
$$

We show that there is an automorphism $\sigma^{*}$ of $\mathfrak{M}^{*}$ fixing $\bigcup_{n \geq 1} A_{n}^{\mathfrak{M}^{*}}$ such that $\sigma(a)=b$.

For the proof of this we require the use of two $\emptyset$-definable functions in $\mathfrak{M}^{*}$. For $x \in M^{*}$ we define $F(x)$ to be the least $y>x$ such that $P(x, y)$, and $G(x)$ to be the greatest $y>x$ such that $P(x, y)$. The functions $F$ and $G$ are easily seen to commute with each other, but on $M^{*} \backslash \bigcup_{n>1} A_{n}^{\mathfrak{M}^{*}}$ there are no relations on iterated applications of $F$ and $G$ that hold beyond those generated by $F \circ G=G \circ F$. Let $\sim$ be the equivalence relation on $M^{*}$ given by $x \sim y$ if and only if there are $i, j \in \mathbb{Z}$ such that $G^{i}(x) \leq y \leq G^{j}(x)$. For each $x \in M^{*}$ we denote by $C_{x}$ the $\sim$-class of $x$. Observe that if $y \notin C_{x}$, then $\neg P(x, y)$.

We now assert that

Claim 2.5.1. For all $d, e \in M^{*} \backslash \bigcup_{n \geq 1} A_{n}^{\mathfrak{M}^{*}}$, there is an isomorphism $\sigma: \mathfrak{M}^{*}\left\lceil C_{d} \cong\right.$ $\mathfrak{M}^{*} \Gamma_{C_{e}}$ such that $\sigma(d)=e$.

Proof. We begin by analyzing $C_{d}$ more closely. Note that if $u \in C_{d}$, then $d \leq$ $G^{i}(u) \leq G(d)$ for some $i \in \mathbb{Z}$. So we now focus on the interval $[d, G(d)] \subset M^{*}$. Let $x \in M^{*}$ satisfy $d \leq x \leq G(d)$. Then $x$ is of one of the following six types:

i. $x=F^{n}(d)$ for some $n \in \omega$;

ii. $x=F^{-n}(G(d))$ for some $n \in \omega$;

iii. $F^{n}(d)<x<F^{n+1}(d)$ for some $n \in \omega$;

iv. $F^{-(n+1)}(G(d))<x<F^{-n}(G(d))$ for some $n \in \omega$;

v. $P(d, x)$ but not (i) or (ii);

vi. $\neg P(d, x)$ but not (iii) or (iv).

Observe that the interval $(d, F(d)) \subset M^{*}$ has order type $\eta$ and that set $\{x \in$ $\left.[d, G(d)]: \mathfrak{M}^{*} \models P(d, x)\right\}$ has order type $\omega+\eta \mathbb{Z}+\omega^{*}$. Note too that if $x$ is of type (vi), then there is some $y \in M^{*}$ of type (v) such that $y<x<F(y)$. Also, if $x \in(d, F(d))$ and $w \in M^{*}$ satisfies $P(d, w)$, then there is a unique $z \in M^{*}$ such that $P(x, z)$ and $z \in(w, F(w))$.

We now are ready to define $\sigma$. Clearly it suffices to define $\sigma$ on $[d, G(d)]$. For each term $t$ - in the language containing $F$ and $G$, of course - we let $\sigma(t(d))=t(e)$. Since $(d, F(d))$ and $(e, F(e))$ both have order type $\eta$, we extend $\sigma$ to $(d, F(d))$ so that it is an order isomorphism. We next extend $\sigma$ so that if $t$ is a term and $x \in(d, F(d))$, then $\sigma(t(x))=t(\sigma(x))$. Thus we have defined $\sigma$ on all elements of types (i)-(iv). As the elements of $C_{d}$ and $C_{e}$ of type (v) have order type $\eta \mathbb{Z}$, we can extend $\sigma$ to the elements of type (v). Lastly, suppose that $z \in C_{d}$ is of type (vi). Then there is an element $w$ of type (v) satisfying $w<z<F(w)$, and we let $x$ be the unique element such that $d<x<F(d)$ and $P(x, z)$. We then define $\sigma(z)$ to be the unique element $u \in(\sigma(w), \sigma(F(w)))$ such that $P(\sigma(x), u)$. One then easily checks that $\sigma$ is the desired isomorphism.

Finally, let $a, b \in M^{*}$ be as in (1) and let $\sigma$ be as in the conclusion of Claim 2.5.1 with $\sigma(a)=b$. We extend $\sigma$ to the desired automorphism $\sigma^{*}$ of $\mathfrak{M}^{*}$ as follows. First, let $\sigma^{*} \uparrow \bigcup_{n \geq 1} A_{n}^{\mathfrak{M}^{*}}$ be the identity. For $w \in M^{*} \backslash \bigcup_{n \geq 1} A_{n}^{\mathfrak{M}^{*}}$ let $[w]_{\sim}$ denote the $\sim$-class of $w$. Observe that the ordering induced on the set of $\sim$-equivalence classes 
of $M^{*} \backslash \bigcup_{n>1} A_{n}^{\mathfrak{M}^{*}}$ has order type $\eta$. Hence there is an order automorphism of the $\sim$-classes which maps $[a]_{\sim}$ to $[b]_{\sim}$. Finally, we combine this order automorphism of the $\sim$-classes with Claim 2.5.1 to extend $\sigma$ to $\sigma^{*}$ on all of $M^{*} \backslash \bigcup_{n \geq 1} A_{n}^{\mathfrak{M}^{*}}$. This completes the definition of $\sigma^{*}$, which is readily seen to be the desired automorphism. Thus we have a contradiction.

2.6. Further Examples. As well-behaved as the model theory of weakly o-minimal structures is, it is not as smooth as that of o-minimal structures. We now present several examples of weakly o-minimal structures to exhibit various pathologies and to make some smaller but interesting points.

Example 2.6.1. Let $\mathfrak{M}=(M,<, P, Q, f)$ be as follows. Let $M$ be the disjoint union of the interpretations of the unary relations $P$ and $Q$, with $P$ preceding $Q$ in the ordering $<$ on $M$. Further, we identify the interpretations of $P$ with $\mathbb{Q}$, ordered as usual, and $Q$ with $\mathbb{Q} \times \mathbb{Q}$, ordered lexicographically. The symbol $f$ is interpreted by a (partial) unary function with $\operatorname{dom}(f)=Q$ and $\operatorname{ran}(f)=P$, and is defined by $f(n, m)=n$ for all $m, n \in \mathbb{Q}$. It is easily checked that $\mathfrak{M}$ is weakly ominimal - in fact all models of $\mathrm{Th} \mathfrak{M}$ are weakly o-minimal. Although the function $f$ is everywhere continuous and locally constant, $\operatorname{dom}(f)$ cannot be partitioned into finitely many convex sets on which $f$ is constant or strictly monotonic. So in general we cannot expect a global monotonicity theorem as for o-minimal structures.

Example 2.6.2. Let $\mathfrak{M}=(M,<, f)$, where $(M,<)$ is naturally identified with $\mathbb{Z} \times \mathbb{Q}$, ordered lexicographically. For any $(z, q) \in M$, let $f(z, q)=(-z, q)$. It can be checked that Th $\mathfrak{M}$ is weakly o-minimal, and that the function $f$ is everywhere locally strictly monotonic, but not piecewise monotonic.

The preceding two examples show that in our treatment of monotonicity in Section 3 we can expect only a local monotonicity theorem for weakly o-minimal structures in general. In Section 6, under the additional assumption that a structure is a non-valuational weakly o-minimal expansion of an ordered field, we obtain a piecewise monotonicity theorem, Proposition 6.4.

We now provide two examples to show that weakly o-minimal theories do not in general have prime models.

Example 2.6.3. Let $\mathfrak{M}=\left(M,<, E_{i}\right)_{i \in \mathbb{N}}$, where $(M,<)$ has order type $(\mathbb{Q},<)$, and for each $n \in \mathbb{N}$ we have that $E_{n}$ is an equivalence relation having the following properties:

i. $E_{0}$ has the single equivalence class $M$.

ii. For each $n \in \mathbb{N}$ the equivalence relation $E_{n+1}$ refines each $E_{n}$-class into infinitely many convex, open classes that are densely ordered without endpoints.

Then $\mathfrak{M}$ is readily seen to have a weakly o-minimal theory. If we expand the language by introducing a unary predicate for each $E_{n}$-class for every $n$, then the resulting theory is weakly o-minimal and does not have a prime model.

Example 2.6.4. Let $\mathfrak{M}=\left(\mathbb{Q},<,+, U_{j}\right)_{j \in J}$, where $J \subset \mathbb{R} \backslash \mathbb{Q}$ is countable and dense, and $U_{j}=(-j, j)$ for each $j \in J$. By an argument like that given in 2.3 or by appealing directly to [3], we see that $\mathrm{Th} \mathfrak{M}$ is weakly o-minimal. It is not difficult to see that this theory does not, however, have a prime model.

We now present an expansion of an ordered field whose theory is weakly ominimal but which does not have prime models. 
Example 2.6.5. Let $(G,<)$ be a divisible ordered abelian group together with a chain $\left(G_{q}\right)_{q \in \mathbb{Q}}$ of proper non-trivial convex subgroups, the chain ordered under inclusion by $\mathbb{Q}$. It is easy to produce such a group; for example, take the Hahn sum of $\mathbb{Q}$ copies of $(\mathbb{Q},+)$. Let $\mathcal{K}:=\mathbb{R}\left[\left[t^{G}\right]\right]$ be the ring of generalized power series with well-ordered support, and expand $\mathcal{K}$ to a structure $\mathcal{K}^{*}$ by endowing it with the natural order and adjoining a binary predicate Div and unary predicates $R$ and $P_{q}$ for $q \in \mathbb{Q}$ as follows:

i. For $f=\sum r_{\gamma} t^{\gamma} \in K$ put $f \in R$ if and only if $\min (\operatorname{supp}(f)) \geq 0$.

ii. For $f \in K$ put $f \in P_{q}$ if and only if $\min (\operatorname{supp}(f)) \in G_{q}$.

iii. The predicate Div is interpreted in the usual way with respect to the valuation ring $R$.

The structure $\left(G,<,+, 0, G_{q}\right)_{q \in \mathbb{Q}}$ is easily seen to admit elimination of quantifiers. It follows from Theorem 5 of [5] that the theory of $\mathcal{K}^{*}$ also has elimination of quantifiers. An expansion of the argument in [6] now shows that $\mathrm{Th} \mathcal{K}^{*}$ is weakly o-minimal, and it can be verified that this theory does not have prime models.

The final example does not exhibit any pathology, but makes a connection with combinatorial work of Cameron [4], and also with the notion of $C$-minimality studied in 19 and 12 .

Example 2.6.6. Let $\mathfrak{M}=(K,<,+, \cdot, R)$ be a real closed field with convex valuation ring $R$. We define a ternary relation $C$ on $K$ by putting $C(x ; y, z)$ if and only if $v(y-x)<v(y-z)$, and let $\mathfrak{N}=(K,<, C)$. The structure $\mathfrak{N}$ is $\omega$-categorical, admits elimination of quantifiers, and is weakly o-minimal 5 This $\omega$-categorical weakly o-minimal structure reveals more complexity than is found in the o-minimal case. Such structures are investigated further in [13], where classification theorems are proved. We note also that the structure $(K, C)$ is $C$-minimal in the sense of [19] and that $\mathfrak{M}$ is $\mathfrak{N}$-minimal in the sense of [19], Section 3 .

\section{Monotonicity}

In this section we prove two monotonicity theorems for definable functions in a weakly o-minimal structure whose domain is a subset of the universe of the structure and whose range is (a subset of) the universe of the structure or a sort in the Dedekind completion of the structure. For these theorems to hold, we require either that all the models of the theory of the structure are weakly o-minimal, or that the structure is an expansion of an ordered group 6 Examples 2.6.1 and 2.6.2 in Section 2 demonstrate that we can expect to obtain only local monotonicity, rather than the piecewise monotonicity of definable functions true for o-minimal structures.

Definition 3.1. Let $f$ be a function from a densely ordered set $I$ to a densely ordered set $K$. We say that $f$ is tidy if one of the following holds:

a. for all $x \in I$ there is an interval $J \subseteq I$ such that $x \in \operatorname{Int}(J)$ and $f \uparrow J$ is strictly increasing, in which case $f$ is said to be locally increasing on $I$;

b. for all $x \in I$ there is an interval $J \subseteq I$ such that $x \in \operatorname{Int}(J)$ and $f \uparrow J$ is strictly decreasing, in which case $f$ is said to be locally decreasing on $I$;

${ }^{5}$ This structure and some related ones arise in a combinatorial context in Section 4 of [4], with the notation $\partial P T$.

${ }^{6}$ In a recent preprint [2] R Arefiev extends Theorems 3.3 and 3.4 by proving, just under the assumption that a structure $\mathfrak{M}$ is weakly o-minimal, that it has monotonicity. 
c. for all $x \in I$ there is an interval $J \subseteq I$ such that $x \in \operatorname{Int}(J)$ and $f \uparrow J$ is constant, in which case $f$ is said to be locally constant on $I$.

Definition 3.2. A weakly o-minimal structure $\mathfrak{M}$ is said to have monotonicity if whenever $A \subseteq \bar{M}$ is a definable sort and $f: D \subset M \longrightarrow A$ is a definable function, there is some $m \in \mathbb{N}$ and a partition of $D=\operatorname{dom}(f)$ into definable sets $X, I_{1}, \ldots, I_{m}$ such that $X$ is finite, each $I_{i}$ is convex, and on each set $I_{i}$ the function $f$ is tidy.

The first major result that we prove in this section is:

Theorem 3.3. If all models of $\mathrm{Th} \mathfrak{M}$ are weakly o-minimal, then $\mathfrak{M}$ has monotonicity.

We also prove the following theorem, in which we only assume weak o-minimality for the given structure but add the hypothesis that the structure is an expansion of an ordered group.

Theorem 3.4. Let $\mathfrak{M}=(M,<,+, 0, \ldots)$ be a weakly o-minimal expansion of an ordered abelian group. Then $\mathfrak{M}$ has monotonicity.

The assumption in Theorem 3.4 that the group is abelian is in fact unnecessary: in Section 5 we prove Theorem 5.1 which says that every weakly o-minimal ordered group is divisible and abelian.

We also observe the following useful proposition, which is an immediate trivial application of weak o-minimality.

Lemma 3.5. If $\mathfrak{M}$ has monotonicity and $f: M \longrightarrow M$ is a definable partial function, then $f$ is continuous on a cofinite subset of its domain.

The next two sections are devoted, respectively, to the proofs of Theorem 3.3 and Theorem 3.4 .

3.1. Proof of Theorem 3.3. Throughout this section-unless specifically noted otherwise - we assume that $\mathfrak{M}=(M,<, \ldots)$ is a structure such that all models of Th $\mathfrak{M}$ are weakly o-minimal. Let $f: D \subset M \longrightarrow A$ be a definable function in $\mathfrak{M}$, where $A$ is a definable sort. We first define the following formulas:

$$
\begin{aligned}
& \phi_{0}(x): \equiv\left(\exists x_{1}>x\right)\left(\forall y \in\left(x, x_{1}\right)\right) f(y)<f(x), \\
& \phi_{1}(x): \equiv\left(\exists x_{1}>x\right)\left(\forall y \in\left(x, x_{1}\right)\right) f(y)=f(x), \\
& \phi_{2}(x): \equiv\left(\exists x_{1}>x\right)\left(\forall y \in\left(x, x_{1}\right)\right) f(y)>f(x), \\
& \psi_{0}(x): \equiv\left(\exists x_{0}<x\right)\left(\forall y \in\left(x_{0}, x\right)\right) f(y)<f(x), \\
& \psi_{1}(x): \equiv\left(\exists x_{0}<x\right)\left(\forall y \in\left(x_{0}, x\right)\right) f(y)=f(x), \\
& \psi_{2}(x): \equiv\left(\exists x_{0}<x\right)\left(\forall y \in\left(x_{0}, x\right)\right) f(y)>f(x) .
\end{aligned}
$$

For $i, j=0,1,2$ we let

$$
\theta_{i j}(x): \equiv \psi_{i}(x) \wedge \phi_{j}(x) .
$$

Observe first by the weak o-minimality of $\mathfrak{M}$ that if $x \in \operatorname{Int}(\operatorname{dom}(f))$, then there are $i, j \in\{0,1,2\}$ such that $\theta_{i j}(x)$ holds. Then, by weak o-minimality of $\mathfrak{M}$ again, there is a partition of $M$ into finitely many points and open convex sets such that each open convex set lies in the solution set of some formula $\theta_{i j}$. To prove Theorem [3.3 we first show that on an open convex set only $\theta_{11}, \theta_{02}$ or $\theta_{20}$ can 
hold - this is accomplished in Lemmas 3.6 and 3.8. If $\theta_{11}$ holds on an open convex set $I$, then it is evident that $f$ is locally constant there. Finally, in Lemma 3.9 it is shown that if $\theta_{02}$ or $\theta_{20}$ holds on an open convex set, then, modulo a finite set, $f$ is 'locally increasing' or 'locally decreasing', respectively, on $I$.

In the lemma below we require only that $\mathfrak{M}$ is weakly o-minimal, not that all models of Th $\mathfrak{M}$ are so.

Lemma 3.6. Let $\mathfrak{M}$ be weakly o-minimal, and let $I \subset M$ be an interval. Then it cannot happen that one of the formulas $\theta_{01}, \theta_{10}, \theta_{12}, \theta_{21}$ holds throughout $I$.

Proof. Suppose that $\theta_{01}$ holds throughout $I$. The other cases are similar, and we omit them. Let $x \in I$. Since $\phi_{1}(x)$ holds, there is some $x_{1}>x$ such that $x_{1} \in I$ and for all $y \in\left(x, x_{1}\right)$ we have $f(x)=f(y)$. Now choose $z \in\left(x, x_{1}\right)$. As $\psi_{0}(z)$ holds, there is some $z_{0}<z$ such that $f(y)<f(z)$ for all $y \in\left(z_{0}, z\right)$. If we then take some $w$ satisfying $\max \left\{x, z_{0}\right\}<w<z$, then $f(w)<f(z)$ and $f(w)=f(x)=f(z)$, which is a contradiction.

For the remainder of this section, the hypothesis that all models of $\mathrm{Th} \mathfrak{M}$ are weakly o-minimal is again in force.

Lemma 3.7. Let $I \subset M$ be an interval and $x \in I$. Then there is some $z \in I$ and a subinterval $J \subset I$ such that

(i) $z>x>J$ or $J>x>z$

and

(ii) for all $y \in J, f(y) \leq f(z)$.

Proof. For $u \in I$ with $u>x$ let

$$
A_{u}:=\{w<x: w \in I \wedge f(w) \leq f(u)\} .
$$

If $A_{u}$ contains a subinterval $I_{0}$ of $I$ for some $u$, then, putting $z:=u$ and $J:=I_{0}$, we obtain the conclusion of the lemma. So suppose that there is no such $u$. By the weak o-minimality of $\mathfrak{M}$, each set $A_{u}$ is finite. Also, as

$$
A_{u} \subseteq A_{u^{\prime}} \Longleftrightarrow f(u) \leq f\left(u^{\prime}\right)
$$

for all $u, u^{\prime} \in I \cap(x, \infty)$, we see that the sets $\left\{A_{u}: u \in I \cap(x, \infty)\right\}$ are linearly ordered by inclusion. Since all models of Th $\mathfrak{M}$ are weakly o-minimal, there is some $N \in \mathbb{N}$ such that $\left|A_{u}\right| \leq N$ for all $u \in I \cap(x, \infty)$. It follows that the set $A:=\bigcup\left(A_{u}: u \in I \cap(x, \infty)\right)$ contains no more than $N$ elements, and hence that there is some $z \in I$ with $z<x$ and $z \notin A$. For all $w \in I \cap(x, \infty)$ we then have $f(w)<f(z)$. By choosing $J$ to be any subinterval of $I \cap(x, \infty)$, we finish the proof.

Lemma 3.8. Neither of the formulas $\theta_{00}$ nor $\theta_{22}$ can hold on an interval.

Proof. We give the proof for $\theta_{22}$; the argument for $\theta_{00}$ is virtually identical. For a contradiction, suppose that $\theta_{22}$ holds on an interval $I_{0}$, and let $x_{0} \in I_{0}$. Replacing $I_{0}$ by a subinterval if necessary, we may assume that $f(y)>f\left(x_{0}\right)$ for all $y \in I_{0} \backslash\left\{x_{0}\right\}$. We now define sequences $\left\langle x_{n}: n<\omega\right\rangle$ and $\left\langle z_{n}: n<\omega\right\rangle$ of elements of $I_{0}$, and sequences $\left\langle I_{n}: n<\omega\right\rangle$ and $\left\langle J_{n}: n<\omega\right\rangle$ of infinite subintervals of $I_{0}$ satisfying the following conditions:

1. $x_{n}, z_{n} \in I_{n}, J_{n} \subseteq I_{n}$, and either $z_{n}>x_{n}>J_{n}$ or $J_{n}>x_{n}>z_{n}$;

2. for all $y \in J_{n}, f(y) \leq f\left(z_{n}\right)$; 
3. $x_{n+1} \in J_{n}$;

4. $x_{n+1} \in I_{n+1}, I_{n+1} \subseteq J_{n}$, and $f(y)>f\left(x_{n+1}\right)$ for all $y \in I_{n+1} \backslash\left\{x_{n+1}\right\}$.

We use Lemma 3.7 to find $z_{n}$ and $J_{n}$ satisfying (1) and (2) at stage $n$, and we obtain $x_{n+1}$ and $I_{n+1}$ exactly as we found $x_{0}$ and $I_{0}$ using $\theta_{22}$. Now let

$$
A_{n}:=\left\{y: f(y)>f\left(x_{n}\right)\right\} .
$$

Claim 3.8.1. $A_{n}$ cannot be written as a union of fewer than $n+2$ convex sets.

Proof of Claim. We show by induction on $m$ that $A_{n} \cap I_{n-m}$ has at least $m+2$ connected components. The induction starts, since for $m=0$ we have $A_{n}=$ $I_{n} \backslash\left\{x_{n}\right\}$, which has the two components $\left\{x \in I_{n}: x<x_{n}\right\}$ and $\left\{x \in I_{n}: x>x_{n}\right\}$.

Now assume as inductive hypothesis that $A_{n} \cap I_{n-(m-1)}$ has at least $m+1$ connected components. There are two cases to consider: $z_{n-m}<x_{n-m}<J_{n-m}$ or $z_{n-m}>x_{n-m}>J_{n-m}$. We suppose that the first alternative holds; the argument in the second case is similar. Observe that $I_{n-(m-1)} \subseteq J_{n-m}$ and so $J_{n-m} \cap A_{n} \neq \emptyset$. As $f\left(z_{n-m}\right) \geq f(y)$ for all $y \in J_{n-m}$, we have $z_{n-m} \in A_{n}$. We also see that $x_{n-m} \notin A_{n}$, since there are points in $I_{n-(m-1)} \backslash A_{n}$, such points lie in $I_{n-m}$, and $f(y)>f\left(x_{n-m}\right)$ for all $y \in I_{n-m}$ with $y \neq x_{n-m}$. It follows that $z_{n-m}$ lies in a connected component of $A_{n} \cap I_{n-m}$ distinct from those meeting $A_{n} \cap I_{n-(m-1)}$. Hence $A_{n} \cap I_{n-m}$ has at least one more connected component than $A_{n} \cap I_{n-(m-1)}$, completing the induction.

Given Claim 3.8.1 the lemma follows immediately from our assumption that all models of Th $\mathfrak{M}$ are weakly o-minimal.

Lemma 3.9. Let $I$ be a non-empty open convex set such that $\theta_{02}(x)$ holds for all $x \in I$. Then there is a definable partition $I=I_{1} \cup \ldots \cup I_{t} \cup X$ such that $X$ is finite, each $I_{j}$ is open and convex, and $f$ is locally increasing on each $I_{j}$. Similarly, if all $x$ in an open convex set I satisfy $\theta_{20}(x)$, the same conclusion holds with 'locally increasing' replaced by 'locally decreasing.'

Proof. Assume that $\theta_{02}(x)$ holds for all $x \in I$. We define the following formulas:

$$
\begin{aligned}
& \chi_{0}(x): \equiv\left(\forall x_{1}>x\right)(\exists y, z)\left[x<y<z<x_{1} \wedge f(z) \leq f(y)\right], \\
& \chi_{2}(x): \equiv\left(\forall x_{1}<x\right)(\exists y, z)\left[x_{1}<y<z<x \wedge f(z) \leq f(y)\right] .
\end{aligned}
$$

Claim 3.9.1. For $i=0,2$, the formula $\chi_{i}$ cannot hold throughout a subinterval of I.

Proof of Claim. We handle the case $i=0$, the case $i=2$ being similar. So assume for a contradiction that $\chi_{0}$ holds throughout a subinterval $I_{0} \subseteq I$. We recursively define sequences $\left\langle x_{n}: n<\omega\right\rangle$ and $\left\langle y_{n}: n<\omega\right\rangle$ of points in $I_{0}$ satisfying

$$
x_{0}<y_{0}<x_{1}<y_{1}<\ldots<x_{n}<y_{n}
$$

and

$$
f\left(x_{0}\right)<f\left(x_{1}\right)<\ldots<f\left(x_{n}\right)<f\left(y_{n}\right)<\ldots<f\left(y_{1}\right)<f\left(y_{0}\right)
$$

for all $n \in \mathbb{N}$. The existence of such sequences is easily seen to violate the assumption that all models of Th $\mathfrak{M}$ are weakly o-minimal, thus yielding the claim. 
So it remains to define the sequences. It is convenient to introduce the auxiliary sequence $\left\langle J_{n}: n\langle\omega\rangle\right.$ of open subintervals of $I_{0}$ which for all $n \in \mathbb{N}$ must satisfy $J_{n}>x_{n}$ and

$$
f\left(x_{n}\right)<f(w)<f\left(y_{n-1}\right) \text { for all } w \in J_{n} .
$$

We let $x_{0} \in I_{0}$. Since $x_{0}$ satisfies $\theta_{02}$, there is an interval $J$ whose left endpoint is $x_{0}$ such that $f\left(x_{0}\right)<f(w)$ for all $w \in J$, and we let $J_{0}:=J$. Now suppose we have defined $x_{i}$ and $J_{i}$ for $i \leq n$, and $y_{i}$ for $i<n$ such that

$$
\begin{gathered}
x_{0}<y_{0}<x_{1}<y_{1}<\ldots<y_{n-1}<x_{n}, \\
f\left(x_{0}\right)<f\left(x_{1}\right)<\ldots<f\left(x_{n}\right)<f\left(y_{n-1}\right)<\ldots<f\left(y_{1}\right)<f\left(y_{0}\right),
\end{gathered}
$$

and for all $i<n$ the properties listed above hold for $J_{i}$. We now define $x_{n+1}, J_{n+1}$, and $y_{n}$. Since $\chi_{0}$ holds throughout $J_{n}$, we may choose $y, z \in J_{n}$ satisfying $y<z$ and $f(z) \leq f(y)$. Let $y_{n}:=y$. Then, as $z$ satisfies $\theta_{02}$, there is an open interval $J \subset J_{n}$ whose right endpoint is $z$ such that $y_{n}<J$ and $f(z)>f(w)$ for all $w \in J$. We now choose $x_{n+1} \in J$. As $x_{n+1}$ satisfies $\theta_{02}$, there is an open subinterval $J_{n+1}$ of $J$ such that $x_{n+1}<J_{n+1}$ and $f\left(x_{n+1}\right)<f(w)$ for all $w \in J_{n+1}$. It is apparent that $x_{n+1}, J_{n+1}$, and $y_{n}$ have the required properties.

Given the claim, it follows by weak o-minimality that $\left\{x \in I: \chi_{0}(x) \vee \chi_{2}(x)\right\}$ is finite. We thus obtain a partition of $I$ into finitely many singletons and open convex sets such that on each convex set $f$ is locally increasing. The proof of the lemma is complete.

Proof of Theorem 3.3. By Lemmas 3.6] and 3.8, there is a partition $\operatorname{dom}(f)=J_{1} \cup$ $\ldots \cup J_{n} \cup Y$ into non-empty convex sets $J_{i}$ for $i=1, \ldots, n$ and a finite set $Y$ such that for each $i=1, \ldots, n$ one of $\theta_{02}, \theta_{11}$, or $\theta_{20}$ holds on $J_{i}$. If $\theta_{02}$ holds on some $J_{i}$, we apply Lemma 3.9 to partition $J_{i}$ further so that the conclusion of the lemma holds on each open convex subset of the refined partition, and we argue similarly if $\theta_{20}$ holds on $J_{i}$. If $\theta_{11}$ holds on $J_{i}$, then it is immediate that $f \uparrow J_{i}$ is locally constant. The theorem is thus proved.

3.2. Proof of Theorem 3.4. We first prove a technical lemma.

Lemma 3.10. Let $\mathfrak{M}$ be a weakly o-minimal structure, let $A$ be a sort in $\bar{M}$, and let $f: M \longrightarrow A$ be a definable partial function. Let $c \in M$ be such that $f(x)>c$ for all $x \in \operatorname{dom}(f)$. Then there is no interval $I \subset M$ such that for every subinterval $J \subset I$, we have that $c$ is a limit point of $\{f(x): x \in J\}$.

Proof. Suppose that there were such $f, c$, and $I$. We apply to $f$ some of the arguments in the proof of Theorem 3.3 , taking care to assume only that $\mathfrak{M}$ is weakly o-minimal. We define the formulas $\phi_{j}(x), \psi_{i}(x), \theta_{i j}(x)$, and $\chi_{i}(x)$ as in Section 3.1. We may clearly assume that the interval $I$ is open, and we may also assume that a fixed formula $\theta_{i j}$ holds throughout $I$.

Claim 3.10.1. The formula $\theta_{00}(x)$ holds for all $x \in I$.

Proof of Claim. Suppose that $\phi_{1}(x) \vee \phi_{2}(x)$ holds for some $x \in I$. Then there is some $z \in I$ with $z>x$ such that $f(y) \geq f(x)$ for all $y \in(x, z)$. Since $f(x)>c$, it follows that $c$ is not a limit point of $\{f(y): y \in(x, z)\}$, a contradiction. Hence $\phi_{0}$ holds throughout $I$. A similar argument shows that $\psi_{0}$ holds throughout $I$ also. 
Now we define

$$
B:=\{x \in I:(\exists y \in I)[y>x \wedge f(y)>f(x)]\} .
$$

By weak o-minimality, either $B$ or $I \backslash B$ contains a final subinterval of $I$, and the argument divides according to these possibilities.

Case 1. Some final subinterval $J$ of $I$ lies in $B$. In this case, there are $b_{0}<b_{1}<$ $b_{2}<\ldots$ in $J$ such that $f\left(b_{i}\right)<f\left(b_{i+1}\right)$ for all $i \in \mathbb{N}$. Also, for each $i \in \mathbb{N}$, since $c$ is a limit point of $\left\{f(x): x \in\left(b_{i}, b_{i+1}\right)\right\}$, there is some $d_{i} \in\left(b_{i}, b_{i+1}\right)$ such that $f\left(d_{i}\right)<f\left(b_{0}\right)$. It follows that $\left\{x \in J: f(x)<f\left(b_{0}\right)\right\}$ is a definable subset of $J$ which is not a finite union of convex sets, contradicting that $\mathfrak{M}$ is weakly o-minimal.

Case 2. Some final subinterval $J$ of $I$ is disjoint from $B$. We may suppose that $J$ is open. Let $y \in J$. By Claim 3.10.1, we have that $y$ satisfies $\theta_{00}$ and hence

$$
\left(\exists y_{0}<y\right)\left(\forall z \in\left(y_{0}, y\right)\right) f(z)<f(y) .
$$

Choosing some such $y_{0} \in J$, we have for all $z \in\left(y_{0}, y\right)$ that $f(z)<f(y)$. This contradicts that $z \in J \subset I \backslash B$.

Proof of Theorem 3.4. We adapt the proof of Theorem 3.3 and use the same notation. So let $f: D \subset M \longrightarrow A$ be a definable function to a definable sort $A$. Changes are needed in two places:

(i) in the proof of Lemma 3.8, since: (a) we apply Claim 3.8.1 to deduce that $\theta_{22}$ cannot hold on an interval and the claim uses Lemma 3.7 for its proof, and (b) to obtain a contradiction from Claim 3.8.1 we use that all models of Th $\mathfrak{M}$ are weakly o-minimal;

(ii) in the proof of Lemma 3.9 since the hypothesis that all models of Th $\mathfrak{M}$ is used at the end of the proof of Claim 3.9.1.

We first handle point (i). We show that for no open interval $I \subset D$ is it the case that every point of $I$ satisfies $\theta_{22}$; that is, every element of $I$ is a local minimum for $f$. For a contradiction, suppose that there is some open interval $I \subset D$ such that every point in $I$ is a local minimum for $f$. For each $x \in I$, define

$$
A_{x}:=\{z-x: z \in I \wedge x<z \wedge \forall y \in(x, z) f(y)>f(x)\}
$$

and let $B:=\left\{\sup A_{x}: x \in I\right\}$. Thus $B$ is a sort in $\bar{M}$. We define a function $g: I \longrightarrow B$ by putting $g(x):=\sup A_{x}$ for every $x \in I$. Since $\phi_{2}$ holds throughout $I$, we have $g(x)>0$ for all $x \in I$. We now assert that for every $a, b \in I$ such that $a<b$, we have that 0 is a limit point of $\{g(x): x \in(a, b)\}$. Indeed, let $\epsilon>0$. As $b$ is a local minimum of $f$, there is a point $c \in(a, b)$ with $b-c<\epsilon$ and $f(c)>f(b)$. It follows that $g(c)<\epsilon$, as required. We thus arrive at a contradiction by Lemma 3.10 and so the conclusion of Lemma 3.8 holds.

We next consider point (ii) above, namely the need to adapt the proof of Lemma 3.9 to the present setting. The essential point is to prove Claim 3.9.1 under the current hypotheses. For a contradiction, suppose that $\theta_{02}(x) \wedge \chi_{0}(x)$ holds for all $x$ in an open interval $I$. We define the sets $A_{x}$, the sort $B$, and the function $g$ exactly as above in our discussion of point (i). Again, as $\phi_{2}$ holds throughout $I$, we have that $g(x)>0$ for all $x \in I$. We claim again that if $a, b \in I$ with $a<b$, then 0 is a limit point of $\{g(x): x \in(a, b)\}$. So let $\epsilon>0$ with $\epsilon<b-a$. As $\chi_{0}(a)$ holds, there are $y, z \in(a, a+\epsilon)$ such that $y<z$ and $f(y) \geq f(z)$. It follows that $g(y)<\epsilon$. Since $y \in(a, b)$, we have proved the assertion. Again, this yields a contradiction by Lemma 3.10. 
Thus we have handled points (i) and (ii), and so the proof of Theorem 3.4 is complete.

\section{Dimension}

In this section we investigate a notion of topological dimension. We then prove a weak form of cell decomposition and establish several useful facts about definable functions whose domain is a subset of $n$-space. We conclude by comparing topological dimension as we define it with an algebraic notion of dimension based on algebraic closure.

4.1. Topological dimension. We begin with our definition of topological dimension.

Definition 4.1. Let $\mathfrak{M}$ be weakly o-minimal and $A \subseteq M^{n}$. The dimension of $X$, denoted $\operatorname{dim}(X)$, is the largest integer $r$ such that there is a projection $\pi: M^{n} \longrightarrow$ $M^{r}$ so that $\pi(A)$ has non-empty interior in $M^{r}$.

We prove by simultaneous induction on $n$ the following two results.

Theorem 4.2. Let $\mathfrak{M}$ be a weakly o-minimal structure with monotonicity, let $X \subseteq$ $M^{n}$ be a definable set with non-empty interior, and let $X:=X_{1} \cup \ldots \cup X_{r}$ be a partition into definable sets. Then at least one of $X_{1}, \ldots, X_{r}$ has non-empty interior.

Theorem 4.3. Let $\mathfrak{M}$ be a weakly o-minimal structure with monotonicity, $B \subset$ $M^{n-1}$ a closed box with non-empty interior, $i \in\{1, \ldots, n\}$, and let $h: B \longrightarrow M$ be continuous. Also let $A$ be a definable sort in $\bar{M}$ and $f: B \longrightarrow A$ a definable function satisfying $f(\bar{x})>h(\bar{x})$ for all $\bar{x} \in B$. Then

$$
\left\{\left(x_{1}, \ldots, x_{i-1}, y, x_{i}, \ldots, x_{n-1}\right): \bar{x}=\left(x_{1}, \ldots, x_{n-1}\right) \in B, h(\bar{x})<y<f(\bar{x})\right\}
$$

has non-empty interior in $M^{n}$.

Before proving these results we note the following corollary.

Corollary 4.4. Let $M$ be a weakly o-minimal structure with monotonicity and let $X, X_{1}, \ldots, X_{r}$ be definable subsets of $M^{n}$ with $X=X_{1} \cup \ldots \cup X_{r}$. Then

$$
\operatorname{dim}(X)=\operatorname{Max}\left\{\operatorname{dim}\left(X_{i}\right): i=1, \ldots, r\right\} .
$$

Proof of Corollary 4.4 We prove the result by induction on $n$. The case $n=$ 1 is trivial. Assume that the result holds for all $m<n$. Clearly $\operatorname{dim}(X) \geq$ $\operatorname{Max}\left\{\left(\operatorname{dim}\left(X_{1}\right), \ldots, \operatorname{dim}\left(X_{r}\right)\right\}\right.$, so it suffices to prove the reverse inequality. We may suppose that $\operatorname{dim}(X)=k<n$, since otherwise the result follows from Theorem 4.2 as we can always arrange that the $X_{i}$ are disjoint. Let $\pi: M^{n} \longrightarrow M^{k}$ be a projection such that $\pi(X)$ has non-empty interior in $M^{k}$. Since $\pi(X)=\bigcup_{i=1}^{r} \pi\left(X_{i}\right)$, by Theorem 4.2 there is some $i$ such that $\pi\left(X_{i}\right)$ has non-empty interior. Then $\operatorname{dim}\left(X_{i}\right) \geq k$, as required.

Proof of Theorems 4.2 and 4.3. The proofs are by simultaneous induction. We refer to the statements of the theorems - with a fixed $N$ in place of $n$-as Theorem $4.2 \mathrm{~N}$ and Theorem $4.3 \mathrm{~N}$, respectively. The first step of the induction is easy, as Theorem 4.2 follows immediately from the definition of weak o-minimality and Theorem 4.3 is trivial. We prove

(1) Theorem $4.2 \mathrm{~N}_{\mathrm{N}-1}+$ Theorem $4.3 \mathrm{H}_{\mathrm{N}} \Longrightarrow$ Theorem $4.2 \mathrm{~N}_{\mathrm{N}}$. 
(2) Theorem $4.2 \mathrm{~N}_{\mathrm{N}-1}+$ Theorem $4.3 \mathrm{~N}_{\mathrm{N}-1} \Longrightarrow$ Theorem $4.3 \mathrm{~h}_{N}$.

Proof of (1). Let $B \subset X$ be a closed box, let $\pi: M^{N} \longrightarrow M^{N-1}$ denote projection onto the first $N-1$ coordinates, and let $a \in M$ be such that there is $\left(a_{1}, \ldots, a_{N-1}, a\right) \in \operatorname{Int}(B)$. For each $\bar{y} \in \pi(\operatorname{Int}(B))$ there is by weak o-minimality a unique $i \in\{1, \ldots, r\}$ and some $b>a$ such that

$$
\{(\bar{y}, x): a<x<b\} \subset X_{i} .
$$

Let $f(\bar{y})$ be the supremum of the set of all such $b$. Then $A:=\{f(\bar{y}): \bar{y} \in \pi(\operatorname{Int}(B))\}$ is a sort in $M$, and $f: M^{N-1} \longrightarrow A$ is a definable partial function. For $i=1, \ldots, r$, colour $\bar{y} \in \pi(\operatorname{Int}(B))$ with colour $c_{i}$ if

$$
\{(\bar{y}, x): a<x<f(\bar{y})\} \subset X_{i} .
$$

By the induction hypothesis, Theorem $4.2{ }_{N-1}$, for some $i=1, \ldots, r$ there is a subset $D$ of $\pi(\operatorname{Int}(B))$ with non-empty interior whose points all are coloured $c_{i}$. Now

$$
\{(\bar{y}, x): \bar{y} \in D \text { and } a<x<f(\bar{y})\} \subset X_{i},
$$

and by Theorem $4.3 \mathrm{~N}$ - with the function $h$ in that theorem taking constant value $a$ - this set has non-empty interior in $M^{N}$.

Proof of (2). For ease of notation assume that $i=N$. For each $\bar{x}=\left(x_{1}, \ldots, x_{N-1}\right)$ $\in B$ and $j=1, \ldots, N-1$, define the partial function $g_{\bar{x}, j}: M \longrightarrow A$ by

$$
g_{\bar{x}, j}(u):=f\left(x_{1}, \ldots, x_{j-1}, u, x_{j+1}, \ldots, x_{N-1}\right),
$$

wherever this makes sense. We color the point $\bar{x} \in B$ with one of $4^{N-1}$ colors, according to whether the function $g_{\bar{x}, j}(u)$ is monotonically increasing, constant, or monotonically decreasing in an open neighbourhood of $x_{j}$, or none of these. Replacing $B$ by a smaller box if necessary, by Theorem $4.2 \mathrm{v}-1$ we may suppose that all elements of $B$ have the same color. In fact - since the set $X$ in Definition 3.2 is finite - it follows that for each $\bar{x} \in B$, each function $g_{\bar{x}, j}(u)$ is monotonic or constant in a neighbourhood of $x_{j}$.

We show next that we can replace the box $B$ by a smaller closed box $B^{\prime}=$ $I_{1} \times \cdots \times I_{N-1}$ such that for each $j$, each function $g_{\bar{x}, j}$ is monotonically increasing on $I_{j}$ for all $\bar{x} \in B^{\prime}$, or monotonically decreasing on $I_{j}$ for all $\bar{x} \in B^{\prime}$, or constant on $I_{j}$ for all $\bar{x} \in B^{\prime}$. We show how to do this for the functions $g_{\bar{x}, N-1}$. The argument is then repeated for the other $j$.

Fix $b \in M$ such that there is a point $\left(d_{1}, \ldots, d_{N-2}, b\right) \in B$ with $\left(d_{1}, \ldots, d_{N-2}, b\right)$ $\in \operatorname{Int}(B)$. Let $C:=\left\{\bar{x} \in M^{N-2}:(\bar{x}, b) \in B\right\}$. For each $\bar{x} \in C$, let $I_{\bar{x}}$ be the maximal convex set containing $b$ on which $g_{\bar{x}, N-1}$ is monotonic or constant. There is a sort $E$ in $\bar{M}$ and a definable function $F: C \longrightarrow E$ so that the convex set $(b, F(\bar{x}))$ is a final segment of $I_{\bar{x}}$ for all $\bar{x} \in C$. Since $C$ has non-empty interior in $M^{N-2}$, we may apply Theorem $4.3 \mathrm{~V}-1$ to obtain $D \subseteq C$ such that

$$
\left\{\bar{x} \in B:\left(x_{1}, \ldots, x_{N-2}\right) \in D \text { and } b<x_{N-1}<F\left(x_{1}, \ldots, x_{N-2}\right)\right\}
$$

has non-empty interior. It follows, after replacing $D$ by a proper subset if necessary, that there is a non-empty open interval $I \subset M$ such that the functions $g_{\bar{x}, N-1}$ are all constant on $I$ for each $\bar{x} \in D \times I$, or all monotonically increasing on $I$ for each $\bar{x} \in D \times I$, or all monotonically decreasing on $I$ for each $\bar{x} \in D \times I$. We now replace $B$ by $D \times I$ and repeat the argument for $j \in\{1, \ldots, N-2\}$. The box obtained at the end is denoted $B^{\prime}$, and has the form $I_{1} \times \cdots \times I_{N-1}$, where $I_{j}$ is the infinite interval $\left[k_{j}, l_{j}\right]$ for each $j=1, \ldots, N-1$. 
Let

$$
S:=\left\{i \in\{1, \ldots, N-1\}: \text { each } g_{\bar{x}, i} \text { is non-decreasing }\right\}
$$

and $T:=\{1, \ldots, N-1\} \backslash S$. For $i=1, \ldots, N-1$, define

$$
\epsilon_{i}:= \begin{cases}l_{i} & \text { if } i \in T \\ k_{i} & \text { if } i \in S\end{cases}
$$

Now let $m:=f\left(\epsilon_{1}, \ldots, \epsilon_{N-1}\right)$. Then $a:=h\left(\epsilon_{1}, \ldots, \epsilon_{N-1}\right)<m$, and $m$ is the least value taken by $f$ on $I_{1} \times \cdots \times I_{N-1}$. Pick $u \in M$ with $a<u<m$. By the continuity of $h$, for $j=1, \ldots, N-1$ there is a closed interval $I_{j}^{\prime}$ such that $\epsilon_{j} \in I_{j}^{\prime} \subseteq I_{j}$, and $h(\bar{x})<u$ for all $\bar{x} \in I_{1}^{\prime} \times \cdots \times I_{N-1}^{\prime}$. Then the interior of the closed box $I_{1}^{\prime} \times \cdots \times I_{N-1}^{\prime} \times[u, m]$ is a non-empty open set lying in

$$
\left\{\left(x_{1}, \ldots, x_{N-1}, y\right): \bar{x} \in B^{\prime} \text { and } h(\bar{x})<y<f(\bar{x})\right\},
$$

as required.

4.2. Cell decomposition. Here we establish a weak version of cell decomposition for those structures whose theories are weakly o-minimal.

Definition 4.5. Let $\mathfrak{M}$ be weakly o-minimal. A cell is a subset of $M^{n}$, where $n>0$, defined as follows.

(1) A 1-cell is a definable convex subset of $M$.

(2) A set $X \subset M^{n+1}$ is an $(n+1)$-cell if there is an $n$-cell $Y \subset M^{n}$ such that

(i) $X=\{(\bar{y}, f(\bar{y})): \bar{y} \in Y\}$, where $f: M^{n} \longrightarrow M$ is a definable function, or

(ii) $X=\left\{(\bar{y}, z): \bar{y} \in Y, f_{1}(\bar{y})<z<f_{2}(\bar{y})\right\}$, where $A_{1}$ and $A_{2}$ are sorts in $\bar{M}$ and $f_{i}: Y \longrightarrow A_{i}$ for $i=1,2$ are definable functions such that $f_{1}(\bar{y})<f_{2}(\bar{y})$ for all $\bar{y} \in Y$. (We allow $\{-\infty\}$ to be the sort $A_{1}$, and $\{+\infty\}$ to be the sort $A_{2}$.)

We frequently use the notation $\left(f_{1}, f_{2}\right)_{Y}$ for a cell given by clause 2(ii).

Theorem 4.6. Let $\mathfrak{M}$ be a model of a weakly o-minimal theory, let $n>0$, and let $X_{1}, \ldots, X_{r}$ be definable subsets of $M^{n}$. Then there is a partition $\mathcal{P}$ of $M^{n}$ into finitely many $n$-cells such that each of $X_{1}, \ldots, X_{r}$ is a union of cells in $\mathcal{P}$.

We call the partition in Theorem 4.6 a decomposition of $M^{n}$ which partitions $X_{1}, \ldots, X_{r}$.

Proof. We use induction on $n$. The result is trivial for $n=1$. Assume now that it is true for $n-1$. Let $\pi: M^{n} \longrightarrow M^{n-1}$ be projection onto the first $n-1$ coordinates. By induction, there is a decomposition of $M^{n-1}$ which partitions each $\pi\left(X_{i}\right)$. So we may assume that $\pi\left(X_{1}\right)=\cdots=\pi\left(X_{r}\right)=Y$, say. For each $\bar{a} \in Y$ let

$$
X_{\bar{a}, i}:=\left\{x \in M:(\bar{a}, x) \in X_{i}\right\} .
$$

Then each $X_{\bar{a}, i}$ is a definable subset of $M$, and so it is the union of a finite number of definable convex sets. Furthermore, the number of such convex sets is bounded as $\bar{a}$ ranges through $Y$, since otherwise there would be an elementary extension of $\mathfrak{M}$ for which infinitely many convex sets were needed, contradicting that $\mathrm{Th} \mathfrak{M}$ is weakly o-minimal. By replacing an interval of the form $[u, v]$ by $\{u\},(u, v),\{v\}-$ and similarly for half-open convex sets - we may assume also that each convex set is a singleton or an open set. By decomposing $Y$ further if necessary, we may suppose that there is some $K \in \mathbb{N}$ such that we have a uniformly definable family of partitions $\mathcal{P}_{\bar{a}}$, for $\bar{a} \in Y$, of $M$ into $K$ convex sets satisfying several conditions: for each $i \in\{1, \ldots, r\}$, if $\bar{a}, \bar{b} \in Y$ then $X_{\bar{a}, i}$ and $X_{\bar{b}, i}$ are unions of the same number 
$n_{i}$ of definable convex sets in $\mathcal{P}_{\bar{a}}$ and $\mathcal{P}_{\bar{b}}$, respectively; that for all $\bar{a} \in Y$ the $j^{\text {th }}$ convex set in $\mathcal{P}_{\bar{a}}$, in increasing order, is of the same type, that is, both or neither are singletons, both or neither have infima in $M$, or in $\bar{M} \backslash M$, or $-\infty$, and similarly for suprema; and lastly that as $\bar{a}$ ranges over $Y$ we have that the $j^{\text {th }}$ convex set in $\mathcal{P}_{\bar{a}}$, in increasing order, is contained in exactly the same sets $X_{\bar{a}, i}$, for $i \in\{1, \ldots, r\}$. It is now clear that for fixed $i \in\{1, \ldots, r\}$ and $j \in\left\{1, \ldots, n_{i}\right\}$, the set of lower bounds of the $j^{\text {th }}$ convex set (in increasing order) in $X_{\bar{a}, i}$, as $\bar{a}$ ranges through $Y$, is a sort $A_{i j}$ in $\bar{M}$. Hence, there is a definable function $f_{i j}: Y \longrightarrow A_{i j}$ that maps each $\bar{a} \in Y$ to the lower bound of the $j^{t h}$ convex set in $X_{\bar{a}, i}$. Upper bounds are handled similarly. The theorem now follows easily.

4.3. Definable functions on $M^{n}$. Our main goal is to prove the following theorem. We thank Bektur Baizhanov and members of his group in Almaty for pointing out an error in the proof in an earlier version of this paper. The Almaty group independently found a correct proof also.

Theorem 4.7. Let $\mathfrak{M}$ be an ordered structure whose theory $\mathrm{Th} \mathfrak{M}$ is weakly ominimal. If $n, m>0$ and $f: D \subset M^{n} \longrightarrow M^{m}$ is a definable bijection between its domain and range, then $\operatorname{dim}(\operatorname{dom}(f))=\operatorname{dim}(\operatorname{ran}(f))$.

Our proof of this result is somewhat indirect, but other useful results emerge in the process. First, we discuss piecewise continuity theorems. One might hope to strengthen Theorem 4.6 by requiring that the functions in Definition 4.5 are continuous. We do not know if this is possible. Problems arise because the range of the function may be a sort in $\bar{M} \backslash M$, and we have not been able to prove a piecewise continuity theorem for definable functions $f: M \longrightarrow A$ when $A$ is a sort (if $A \subseteq M$ then we do have piecewise continuity, by Lemma 3.5). We can, however, salvage the following result.

Theorem 4.8. Let $\mathfrak{M}$ be an ordered structure whose theory $\mathrm{Th} \mathfrak{M}$ is weakly ominimal. If $n>0$ and $g: M^{n} \longrightarrow M$ is a definable partial function such that $\operatorname{dom}(g)$ has non-empty interior, then there is an open $U \subset \operatorname{dom}(g)$ on which $g$ is continuous.

Proof. We use induction on $n$. By Theorem 3.3 and Lemma 3.5 the result holds for $n=1$. Assume that it is true for all $m<n$. Define

$$
X_{1}:=\left\{(\bar{a}, b) \in \operatorname{dom}(g) \mid \begin{array}{l}
\text { there is an open box } A \subset M^{n-1} \\
\text { containing } \bar{a} \text { such that } g(\bar{x}, b) \text { is continuous on } A
\end{array}\right\}
$$

and

$$
X_{2}:=\left\{\begin{array}{l|l}
(\bar{a}, b) \in \operatorname{dom}(g) & \begin{array}{l}
\text { there is an open convex } B \subset M \text { with } \\
b \in B \text { and } g(\bar{a}, y) \text { continuous and either } \\
\text { constant or monotonic on } B
\end{array}
\end{array}\right\} .
$$

For $(\bar{a}, b) \in X_{2}$ let $B_{(\bar{a}, b)}$ be the maximal convex set $B$ in the definition of $X_{2}$. For $i=1,2$ let $h_{i}: X_{2} \longrightarrow \bar{M}$ be defined for $(\bar{a}, b) \in X_{2}$ by $h_{1}(\bar{a}, b)=\inf \left(B_{(\bar{a}, b)}\right)$ and $h_{2}(\bar{a}, b)=\sup \left(B_{(\bar{a}, b)}\right)$. So $\left\{h_{i}(\bar{a}, b):(\bar{a}, b) \in X_{2}\right\}$ is a sort in $\bar{M}$ for $i=1,2$. Now define $X_{3}$ to be the set of all $(\bar{a}, b) \in X_{2}$ such that there is an open convex $B$ containing $b$ and an open box $A \subset M^{n-1}$ containing $\bar{a}$ such that

$$
h_{1}(\bar{x}, b)<\inf (B)<\sup (B)<h_{2}(\bar{x}, b)
$$


for all $\bar{x} \in A$. By Theorem 4.6, there is a decomposition of dom $(g)$ into $n$-cells which partitions $X_{1}, X_{2}$, and $X_{3}$. Let $C$ be a cell in this decomposition which has non-empty interior, as guaranteed by Theorem 4.2, and let $U \subset C$ be an open box.

Claim 4.8.1. $U \subset X_{1} \cap X_{2} \cap X_{3}$.

Proof of Claim. To see that $U \subset X_{1}$, let $(\bar{a}, b) \in U$ and put $U^{\prime}:=\left\{\bar{x} \in U: x_{n}=b\right\}$. Let $\pi: M^{n} \longrightarrow M^{n-1}$ denote projection onto the first $n-1$ coordinates and let $U^{\prime \prime}=\pi\left(U^{\prime}\right)$. Then $g(\bar{x}, b): U^{\prime \prime} \longrightarrow M$ is a definable function whose domain has non-empty interior, so by induction there exists an open box $D \subseteq U^{\prime \prime}$ on which it is continuous. Now choose $\bar{a}^{\prime} \in D$. By the definition of $X_{1}$, we see that $\left(\bar{a}^{\prime}, b\right) \in X_{1}$. Since also $\left(\bar{a}^{\prime}, b\right) \in U$, we have $U \cap X_{1} \neq \emptyset$. Hence, as $C$ is a cell in a decomposition of $X_{1}, X_{2}$, and $X_{3}$, we conclude that $U \subseteq X_{1}$.

The proof that $U \subset X_{2}$ is similar and uses Lemma 3.5. We now show that $U \subset X_{3}$. Fix $(\bar{a}, b) \in U$. Since $U \subset X_{2}$, we have $h_{1}(\bar{x}, b)<b<h_{2}(\bar{x}, b)$ for all $\bar{x}$ such that $(\bar{x}, b) \in U$. By Theorem 4.3, there is an open box $A \subset M^{n-1}$ and $c_{1}, c_{2} \in M$ with $c_{1}<b<c_{2}$ such that for all $\bar{x} \in A$ and $y \in\left[c_{1}, c_{2}\right]$ we have $(\bar{x}, y) \in U$ and $h_{1}(\bar{x}, b)<y<h_{2}(\bar{x}, b)$. Hence, for every $\bar{a}^{\prime} \in A$ we have $\left(\bar{a}^{\prime}, b\right) \in U \cap X_{3}$, and so $U \cap X_{3} \neq \emptyset$. As above, we conclude that $U \subset X_{3}$, which yields the claim.

We now prove that $g$ is continuous on $U$. Let $(\bar{a}, b) \in U$, and let $J$ be an open interval containing $g(\bar{a}, b)$. As $(\bar{a}, b) \in X_{3}$, there exist a box $A \subset M^{n-1}$ and an open convex $B \subset M$ with $\bar{a} \in A$ and $b \in B$ as provided in the definition of $X_{3}$. We clearly may take $A$ and $B$ satisfying $A \times B \subset U$ also. Since $(\bar{a}, b) \in X_{2}$, there are $b_{1}, b_{2} \in B$ such that $b_{1}<b<b_{2}$ and $g\left(\bar{a}, b_{1}\right), g\left(\bar{a}, b_{2}\right) \in J$. Lastly, as $U \subseteq X_{1}$, there are open boxes $A_{1}, A_{2} \subset M^{n-1}$ containing $\bar{a}$ such that $g\left(\bar{x}, b_{1}\right) \in J$ for all $\bar{x} \in A_{1}$, and $g\left(\bar{x}, b_{2}\right) \in J$ for all $\bar{x} \in A_{2}$. It is now easy to see that $\left(A \cap A_{1} \cap A_{2}\right) \times\left(b_{1}, b_{2}\right)$ is an open box containing $(\bar{a}, b)$ whose image lies in $J$, as required.

Lemma 4.9. Let $\mathfrak{M}$ be an $\omega_{1}$-saturated model of a weakly o-minimal theory, and let $U_{0} \supseteq U_{1} \supseteq \cdots \supseteq U_{i} \supseteq \cdots$ for $i \in \mathbb{N}$ be definable subsets of $M^{n}$ such that for all $\bar{a}=\left(a_{1}, \ldots, a_{n}\right) \in U=\bigcap_{i \in \mathbb{N}} U_{i}$ and $j \in\{1, \ldots, n\}$, there are $c_{1}<a_{j}<c_{2}$ such that $\left(a_{1}, \ldots, a_{j-1}, x, a_{j+1}, \ldots, a_{n}\right) \in U$ for all $x \in\left(c_{1}, c_{2}\right)$. Then $U$ has non-empty interior in $M^{n}$.

Proof. Let $r \leq n$ be maximal such that (after reordering the coordinates if necessary) there exist an open box $B \subseteq M^{r}$ and $a_{r+1}, \ldots, a_{n} \in M$ such that $\left(\bar{x}, a_{r+1}, \ldots, a_{n}\right) \in U$ for all $\bar{x} \in B$. Suppose for a contradiction that $r<n$.

Let $i \in \mathbb{N}$. For each $\bar{x} \in B$ let $\left(h_{1}^{i}(\bar{x}), h_{2}^{i}(\bar{x})\right)$ be the largest convex set containing $a_{r+1}$ such that $\left(\bar{x}, y, a_{r+2}, \ldots, a_{n}\right) \in U_{i}$ for every $y \in\left(h_{1}^{i}(\bar{x}), h_{2}^{i}(\bar{x})\right)$. So $h_{1}^{i}, h_{2}^{i}$ are definable functions with range in a sort. By Theorem 4.3 there is a box $B_{i} \subset U_{i}$ of dimension $r+1$, that is, a box $B_{i}=J_{1} \times \ldots \times J_{n}$ where some $r+1$ of the $J_{1}, \ldots, J_{n}$ are open intervals and the remaining $n-(r+1)$ are singleton subsets of $M$. Then the $\omega_{1}$-saturation of $\mathfrak{M}$ implies that there exists an open box $B^{*} \subset U$ of dimension $r+1$, contradicting the maximality of $r$.

Theorem 4.10. Let $\mathfrak{M}$ be a model of a weakly o-minimal theory, let $n>0$, and let $f: M^{n} \longrightarrow M^{n}$ be a definable injective partial function whose domain has non-empty interior. Then $\operatorname{ran}(f)$ has non-empty interior.

Proof. By taking an elementary extension if necessary, we may assume that $\mathfrak{M}$ is $\omega_{1}$-saturated. The proof is by induction on $n$. For $n=1$ the result is trivial. 
Assume that the theorem holds for all $m<n$, that $n>1$, and that $f$ is as in the theorem. By Theorem 4.8 we may suppose that $f$ is continuous. We also may assume that $\operatorname{dom}(f)$ is an open box.

Observe that if for each $\bar{b}=\left(b_{1}, \ldots, b_{n}\right) \in \operatorname{ran}(f)$ and $j \in\{1, \ldots, n\}$ there is an open interval $J$ containing $b_{j}$ such that

$$
\left(b_{1}, \ldots, b_{j-1}, y, b_{j+1} \ldots, b_{n}\right) \in \operatorname{ran}(f) \text { for all } j \in J,
$$

then it follows by Lemma 4.9 that $\operatorname{ran}(f)$ has interior. Bearing this in mind, we partition $\operatorname{ran}(f)$ into the definable sets $Y_{0}^{0}, \ldots, Y_{n}^{0}$ as follows. If $\bar{b}=\left(b_{1}, \ldots, b_{n}\right) \in$ $\operatorname{ran}(f)$ is such that for some $j \in\{1, \ldots, n\}$ there is no interval $J$ containing $b_{j}$ satisfying $(*)$, we put $\bar{b} \in Y_{j}^{0}$ if $j$ is least for which this occurs; otherwise we put $\bar{b} \in Y_{0}^{0}$. By Theorem 4.2 at least one of the definable sets $f^{-1}\left(Y_{0}^{0}\right), \ldots, f^{-1}\left(Y_{n}^{0}\right)$ has non-empty interior. We examine first the case that none of $f^{-1}\left(Y_{1}^{0}\right), \ldots, f^{-1}\left(Y_{n}^{0}\right)$ has non-empty interior.

Let $B_{0}$ be an open box contained in $f^{-1}\left(Y_{0}^{0}\right)$. We consider $f \uparrow_{B_{0}}$. Let $\bar{b}=$ $\left(b_{1}, \ldots, b_{n}\right) \in \operatorname{ran}\left(f \uparrow_{B_{0}}\right)$. Although for each $j \in\{1, \ldots, n\}$ there is an open interval $J$ containing $b_{j}$ for which $(*)$ holds, this may no longer be the case if in (*) we replace $\operatorname{ran}(f)$ by $\operatorname{ran}\left(f \uparrow_{B_{0}}\right)$. We thus repeat the steps above to partition $\operatorname{ran}\left(f \uparrow_{B_{0}}\right)$ into the definable sets $Y_{0}^{1}, \ldots, Y_{n}^{1}$, and again consider the case that none of $f^{-1}\left(Y_{1}^{1}\right), \ldots, f^{-1}\left(Y_{n}^{1}\right)$ has interior. Continuing, we obtain a decreasing sequence $Y_{0}^{0} \supset Y_{0}^{1} \supset Y_{0}^{2} \supset \cdots$ of subsets of $\operatorname{ran}(f)$ and set $Y=\bigcap_{k \in \mathbb{N}} Y_{0}^{k} \subset \operatorname{ran}(f)$. By saturation we have $Y \neq \emptyset$, and we put $X:=f^{-1}(Y)$. Using the $\omega_{1}$-saturation of $\mathfrak{M}$ once more, it is easy to verify that for all $\bar{b}=\left(b_{1}, \ldots, b_{n}\right) \in Y$ and $j=1, \ldots, n$ there is an interval $J$ containing $b_{j}$ satisfying $(*)$ with $f$ replaced by $f \uparrow_{X}$. Applying Lemma 4.9. we conclude that $\operatorname{ran}\left(f \uparrow_{X}\right)$ and hence $\operatorname{ran}(f)$ has interior.

We have thus reduced to the case that for some $k \in \mathbb{N}$ and $j \in\{1, \ldots, n\}$ the set $f^{-1}\left(Y_{j}^{k}\right)$ has interior. Let $B$ be an open box contained in $f^{-1}\left(Y_{j}^{k}\right)$. Notice that for $\bar{b}=\left(b_{1}, \ldots, b_{n}\right) \in \operatorname{ran}\left(f \uparrow_{B}\right)$ it is still the case that there is no open interval $J$ containing $b_{j}$ satisfying $(*)$ with $f$ replaced by $f \uparrow_{B}$. We now replace $f$ by $f \uparrow_{B}$ and for notational convenience assume that $i=n$.

By weak o-minimality we see for all $\bar{b} \in \operatorname{ran}(f)$ that $\left\{x \in M:\left(b_{1}, \ldots, b_{n-1}, x\right) \in\right.$ $\operatorname{ran}(f)\}$ is finite. Moreover, as $\mathfrak{M}$ is a model of a weakly o-minimal theory, there is an uniform bound on the size of all such sets. Partitioning $\operatorname{dom}(f)$ further if necessary and applying Theorem 4.2, we may suppose also for all $\bar{y}=\left(y_{1}, \ldots, y_{n}\right) \in \operatorname{ran}(f)$ that

$$
\left|\left\{z:\left(y_{1}, \ldots, y_{n-1}, z\right) \in \operatorname{ran}(f)\right\}\right|=1 .
$$

Let $\pi: M^{n} \longrightarrow M^{n-1}$ denote projection onto the first $n-1$ coordinates, and let $I$ be the image of $B$ under projection of $M^{n}$ onto the last coordinate. For each $a \in I$ define $g_{a}: \pi(B) \longrightarrow M^{n-1}$ by putting $g_{a}(\bar{x})=\pi(f(\bar{x}, a))$ for all $\bar{x} \in \pi(B)$. By $(* *)$ we see that each such function $g_{a}$ is injective. Since the domain of each $g_{a}$ is an open box, by induction we see that $\operatorname{ran}\left(g_{a}\right)$ has non-empty interior in $M^{n-1}$ for all $a \in I$.

Now fix $a \in I$. Choose $\bar{d} \in \pi(B)$ such that $g_{a}(\bar{d}) \in \operatorname{Int}\left(\operatorname{ran}\left(g_{a}\right)\right)$ and hence such that there is an open box $C \subseteq M^{n-1}$ with $g_{a}(\bar{d}) \in C \subseteq \operatorname{ran}\left(g_{a}\right)$. Let $f(\bar{d}, a)=$ $\left(g_{a}(\bar{d}), e\right)$, and let $J$ be an open interval containing $e$. Since $f$ is continuous, there is an open box $D \subset M^{n}$ containing $(\bar{d}, a)$ such that $f(D) \subseteq C \times J$. We now choose $a^{\prime} \neq a$ such that $\left(\bar{d}, a^{\prime}\right) \in D$. Let $f\left(\bar{d}, a^{\prime}\right)=\left(\bar{c}, e^{\prime}\right)$, where $\bar{c} \in C$ and $e^{\prime} \in J$. Since $C \subseteq \operatorname{ran}\left(g_{a}\right)$ there exist $\bar{d}^{\prime} \in \operatorname{dom}\left(g_{a}\right)$ and $e^{\prime \prime} \in M$ so that $f\left(\bar{d}^{\prime}, a\right)=\left(g_{a}\left(\bar{d}^{\prime}\right), e^{\prime \prime}\right)=$ 
$\left(\bar{c}, e^{\prime \prime}\right)$. As $f$ is injective, we have $e^{\prime} \neq e^{\prime \prime}$. Hence

$$
|\{z \in M:(\bar{c}, z) \in \operatorname{ran}(f)\}| \geq 2,
$$

contradicting $(* *)$.

We now prove a result which makes it possible to strengthen the notion of a cell: it enables us to require in a cell decomposition that every cell has a homeomorphic projection onto an open set. However, the boundary functions of cells clearly need not be continuous.

Theorem 4.11. Let $\mathfrak{M}$ be a model of a weakly o-minimal theory, let $n>0$, and let $X$ be a definable subset of $M^{n}$. Then there is a definable partition of $X$ into cells $C_{1}, \ldots, C_{r}$ such that for each $i=1, \ldots, r$ there exist $t_{i} \leq n$ and a projection $\pi_{i}: M^{n} \longrightarrow M^{t_{i}}$ such that $\pi_{i}\left(C_{i}\right)$ is open in $M^{t_{i}}$ and $\pi_{i}: C_{i} \longrightarrow \pi_{i}\left(C_{i}\right)$ is a homeomorphism.

Proof. We assume that $\mathfrak{M}$ is $\omega_{1}$-saturated - the reader can easily verify that this is harmless, as any parameters that appear are seen to be definable over the parameters used in the definition of $X$. The proof of the theorem is by induction on $n$. For $n=1$, the result is immediate. Assume $n>1$, and that the result holds for all $m<n$. By Theorem 4.6] we may suppose that $X$ is an $n$-cell. Let $\pi_{n}: M^{n} \longrightarrow M^{n-1}$ denote projection onto the first $n-1$ coordinates. By induction, we may suppose that either $\pi_{n}(X)$ is an open cell or $\pi_{n}(X)$ admits a homeomorphic projection $\pi^{*}$ onto an open cell in $M^{k}$ for some $k<n-1$. In the interest of simplifying notation, we let $\pi^{*}$ be the identity map in the first case and then let $\pi:=\pi^{*} \circ \pi_{n}$. So we may suppose that $\pi(X)$ is an open cell in $M^{k}$ for some $k \leq n-1$.

Suppose first that there is a definable function $f: \pi(X) \longrightarrow M$ such that $X:=\left\{\left(\left(\pi^{*}\right)^{-1}(\bar{y}), f(\bar{y})\right): \bar{y} \in \pi(X)\right\}$. Let

$$
U:=\{\bar{x} \in \pi(X): f \text { is continuous in a neighbourhood of } \bar{x}\} .
$$

By Theorem 4.6 there is a decomposition of $\{U, \pi(X) \backslash U\}$ into $k$-cells. Suppose first that $C$ is a cell in this decomposition such that $C \cap U=\emptyset$. By Theorem 4.8 . in this case $C$ must have empty interior, and by the induction hypothesis $C$ has a homeomorphic projection onto an open cell $C^{\prime}=\pi^{\prime}(C) \subset M^{k^{\prime}}$, where $k^{\prime}<n-1$. Let $h: C^{\prime} \longrightarrow M$ be defined by $h=f \circ \pi^{\prime-1}$. As the graph of $h$, a subset of $M^{k^{\prime}+1}$, is homeomorphic to the graph of the restriction to $\pi^{-1}(C)$ and $k^{\prime}+1 \leq n-1$, the induction hypothesis enables us to finish in this case. Now suppose that $C$ is a cell in the decomposition satisfying $C \subset U$. So $f$ is continuous throughout $C$. It then follows easily that $\pi$ is a homeomorphism, and this completes the argument if $X$ is the graph of a definable function.

Suppose now that there are definable functions $f_{i}: M^{n-1} \longrightarrow A_{i}$ for $i=1,2$, where the $A_{i}$ are sorts, such that $f_{1}(\bar{x})<f_{2}(\bar{x})$ for all $\bar{x} \in \pi_{n}(X)$ and

$$
X:=\left(f_{1}, f_{2}\right)_{\pi_{n}(X)} .
$$

Suppose first that $\pi(X)$ is an open cell in $M^{k}$ for some $k<n-1$. If we let $h_{i}=f_{i} \circ\left(\pi^{*}\right)^{-1}$ for $i=1,2$, we see that $X$ is homeomorphic to $\left(h_{1}, h_{2}\right)_{\pi(X)} \subset M^{k+1}$, and as $k+1 \leq n-1$ we finish by induction. We thus are left with the case that $k=n-1$. So for the remainder of the proof $\pi(X)$ is an open cell in $M^{n-1}$.

For each $\bar{a} \in \pi(X)$ let $X_{\bar{a}}:=\left(f_{1}(\bar{a}), f_{2}(\bar{a})\right)$, and let $\widetilde{X}_{\bar{a}}:=\left\{b \in X_{\bar{a}}:(\bar{a}, b) \in\right.$ $\operatorname{Int}(X)\}$. For each $\bar{a} \in \pi(X)$, observe that if $\widetilde{X}_{\bar{a}} \neq \emptyset$, then $\tilde{X}_{\bar{a}}$ is an open convex 
subset of $M$. By decomposing $\pi(X)$ further if necessary, we may suppose that $\widetilde{X}_{\bar{a}}=\emptyset$ for all $\bar{a} \in \pi(X)$ or $\widetilde{X}_{\bar{a}} \neq \emptyset$ for all $\bar{a} \in \pi(X)$. In the first case, we see that $\operatorname{Int}(X)=\emptyset$. In the second case, define $h_{i}: \pi(X) \longrightarrow \bar{M}$ for $i=1,2$ by $h_{1}(\bar{a})=\inf \widetilde{X}_{\bar{a}}$ and $h_{2}(\bar{a})=\sup \widetilde{X}_{\bar{a}}$. Notice that $\operatorname{Int}(X)=\left(h_{1}, h_{2}\right)_{\pi(X)}$, and so $\operatorname{Int}(X)$ is an open cell in $M^{n}$ in this case. Replacing $X$ by $X \backslash \operatorname{Int}(X)$ if necessary, we consequently may assume for the remainder of the proof that $X$ has empty interior.

To summarize, we have reduced the proof to the situation when $X=\left(f_{1}, f_{2}\right)_{\pi(X)}$ is an $n$-cell with empty interior and $\pi(X)$ is an open cell in $M^{n-1}$. A point $(\bar{a}, b) \in X$ is called $X$-good if for all $i \in\{1, \ldots, n-1\}$, there are $c_{1}<a_{i}<c_{2}$ depending on $\bar{a}$ and $i$ such that for all $y \in\left(c_{1}, c_{2}\right)$ we have

$$
f_{1}\left(a_{1}, \ldots, a_{i-1}, y, a_{i+1}, \ldots, a_{n-1}\right)<b<f_{2}\left(a_{1}, \ldots, a_{i-1}, y, a_{i+1}, \ldots, a_{n-1}\right) .
$$

Notice that if $\left(\bar{a}, b_{1}\right)$ and $\left(\bar{a}, b_{2}\right)$ are $X$-good, then $(\bar{a}, d)$ is $X$-good for all $b_{1}<d<b_{2}$. We see also that if $(\bar{a}, b)$ is $X$-good, then there are $b_{1}, b_{2} \in M$ such that $b_{1}<b<b_{2}$ and each of $\left(\bar{a}, b_{1}\right)$ and $\left(\bar{a}, b_{2}\right)$ is $X$-good. This follows from the observation that if $i \in\{1, \ldots, n-1\}$ and $c_{1}, c_{2}$ are as in the definition of $X$-goodness, then we may suppose by Theorem 3.3 and Corollary 4.4, after partitioning $\pi(X)$ further if necessary, that the functions $f_{i}\left(a_{1}, \ldots, a_{i-1}, x, a_{i+1}, \ldots, a_{n-1}\right)$ are constant or strictly monotonic on $\left(c_{1}, c_{2}\right)$. Hence for each $\bar{a} \in \pi(X)$, the set

$$
X_{\bar{a}}^{g}=\{b \in M:(\bar{a}, b) \in X \text { and is } X \text {-good }\},
$$

if non-empty, is infinite, convex, and open.

Let $W=\left\{\bar{a} \in \pi(X): X_{\bar{a}}^{g} \neq \emptyset\right\}$. So $W$ is definable. For each $\bar{a} \in W$, the set $X_{\bar{a}}^{g}$ has infimum $h_{1}(\bar{a})$ and supremum $h_{2}(\bar{a})$, say, where these are sorts in $\bar{M}$. Thus

$$
f_{1}(\bar{a}) \leq h_{1}(\bar{a})<h_{2}(\bar{a}) \leq f_{2}(\bar{a})
$$

for each $\bar{a} \in W$. We now define the $n$-cell $X_{1}:=\left(h_{1}, h_{2}\right)_{W}$. By the remarks in the preceding paragraph, we note that $X_{1}$ is precisely the set of $X$-good points.

We now let $X_{0}=X$, let $X_{1}$ be as before the set of $X$-good points, and in general for $j \geq 1$ define $X_{j+1}$ to be the set of $X_{j}$-good points. As above, for $j \geq 1$ we let $W_{j}=\left\{\bar{a} \in \pi\left(X_{j-1}\right):\left(X_{j-1}\right)_{\bar{a}}^{g} \neq \emptyset\right\}$ and have $X_{j}=\left(h_{1}^{j}, h_{2}^{j}\right)_{W_{j}}$ for definable functions $h_{1}^{j}$ and $h_{2}^{j}$ taking values in a sort in $\bar{M}$. Note that $h_{1}^{j} \leq h_{1}^{j+1}$ and $h_{2}^{j+1} \leq h_{2}^{j}$ for all $j$. We now wish to prove

Claim 4.11.2. For some $j \geq 1$ the definable set $W_{j}$ has empty interior in $\pi\left(M^{n}\right)$.

Proof of Claim. For a contradiction, suppose that this is not the case. By $\omega_{1}$ saturation there is an open box $B \subset \bigcap_{j \in \mathbb{N}} W_{j}$. Also, $\omega_{1}$-saturation implies for all $\bar{a} \in B$ that $\bar{a} \in \pi\left(\bigcap_{j \in \mathbb{N}} X_{j}\right)$. For this, note that each non-empty $\left(X_{j}\right)_{\bar{a}}^{g}$ is an infinite convex set and that $\left(X_{j}\right)_{\bar{a}}^{g} \supseteq\left(X_{j+1}\right)_{\bar{a}}^{g}$ for all $j$.

We now assert that for all $\left(\bar{a}, a_{n}\right) \in(B \times M) \cap \bigcap_{j \in \mathbb{N}} X_{j}$ and each $i=1, \ldots, n$ there are $c_{1}, c_{2} \in M$ with $c_{1}<a_{i}<c_{2}$ such that $\left(a_{1}, \ldots, a_{i-1}, y, a_{i+1}, \ldots, a_{n}\right) \in$ $\bigcap_{j \in \mathbb{N}} X_{j}$ for all $y \in\left(c_{1}, c_{2}\right)$. For $i \leq n-1$ this follows from $\omega_{1}$-saturation, using the fact that for each fixed $j$ there are $c_{1}, c_{2} \in M$ with $c_{1}<a_{i}<c_{2}$ satisfying

$$
h_{1}^{j}\left(a_{1}, \ldots, a_{i-1}, y, a_{i+1}, \ldots, a_{n-1}\right)<a_{n}<h_{2}^{j}\left(a_{1}, \ldots, a_{i-1}, y, a_{i+1}, \ldots, a_{n-1}\right)
$$

for all $y \in\left(c_{1}, c_{2}\right)$. For $i=n$ saturation implies that our assertion holds given that $\left(X_{j}\right)_{\bar{a}}^{g} \supseteq\left(X_{j+1}\right)_{\frac{g}{a}}$ for all $j$, and that if $\left(X_{j}\right)_{\bar{a}}^{g} \neq \emptyset$ then $\left(X_{j}\right)_{\bar{a}}^{g}$ is an open convex set. 
By Lemma 4.9 we conclude that $\bigcap_{j \in \mathbb{N}} X_{j}$ has non-empty interior in $M^{n}$. As $X \supseteq \bigcap_{j \in \mathbb{N}} X_{j}$ and we have assumed that $X$ has empty interior in $M^{n}$, this is a contradiction.

By Claim 4.11.2 we thus may assume for some $j_{0}$ that $W_{j_{0}}$ has empty interior in $\pi\left(M^{n}\right)$. By decomposing $\pi(X)$ further if necessary and applying induction, we thus may suppose that all points of $X$ are not $X$-good. By decomposing again if necessary we also may assume that all points of $X$ are not $X$-good with respect to the same coordinate $i$. Let $\rho_{i}: M^{n} \longrightarrow M^{n-1}$ be the projection which drops the $i^{t h}$ coordinate. It follows that $\rho_{i}^{-1}(\bar{b})$ is finite for each $\bar{b} \in \rho_{i}(X)$. Since all models of Th $\mathfrak{M}$ are weakly o-minimal, there is a uniform finite bound for $\rho_{i}^{-1}(\bar{b})$ for $\bar{b} \in \rho_{i}(X)$. Since this situation reduces to that of the graph of a function $F: D \subset M^{n-1} \longrightarrow M$ and we already have dealt with this case, the proof of the theorem is complete.

Proof of Theorem 4.7. Let $f, n$, and $m$ be as in the statement of the theorem. Also, let $X:=\operatorname{dom}(f), r:=\operatorname{dim}(X)$, and $s:=\operatorname{dim}(f(X))$. Since $f$ is a bijection, it suffices to prove $r \geq s$. By Theorem 4.11 and Corollary 4.4 we may suppose that there is a homeomorphic projection $\pi$ of $\operatorname{dom}(f)$ onto an open $r$-cell $U \subseteq M^{r}$. Then $f \circ \pi^{-1}: U \longrightarrow \operatorname{ran}(f)$ is a definable bijection. There also exist a cell $C \subset f(X)$ and a projection $\rho: M^{m} \longrightarrow M^{s}$ such that $\rho$ projects $C$ bijectively onto an open subset of $M^{s}$. For a contradiction, suppose that $r<s$. Choose $a_{1}, \ldots, a_{s-r} \in M$, let

$$
W:=\left\{\left(\bar{x}, a_{1}, \ldots, a_{s-r}\right): f \circ \pi^{-1}(\bar{x}) \in C\right\},
$$

and define $\sigma: U \subset M^{r} \longrightarrow M^{s}$ by $\sigma(\bar{x})=\left(\bar{x}, a_{1}, \ldots, a_{s-r}\right)$. Then $\sigma \circ \pi \circ f^{-1} \circ \rho^{-1}$ is a definable bijection from an open subset of $M^{s}$ onto the set $W \subset M^{s}$ with empty interior in $M^{s}$, contradicting Theorem 4.10.

4.12. Topological and algebraic dimension. As noted in Section 2.4 and Example 2.6.1, there are weakly o-minimal structures with monotonicity such that algebraic closure does not have the exchange property (the example in Section 2.4 has monotonicity by Theorem 3.4. In this subsection we examine weakly o-minimal theories such that algebraic closure has the exchange property for all models of the theory.

For a model $\mathfrak{M}$ of such a theory and $A \subseteq M$, we can define $\operatorname{Dim}(A)$ - as distinct from $\operatorname{dim}(A)$ given by Definition 4.1 to be the cardinality of any maximal algebraically independent subset of $A$. This is well-defined, by the exchange property. If $B \subseteq M$, then we can define $\operatorname{Dim}(A / B):=\operatorname{Dim}(A \cup B)-\operatorname{Dim}(B)$.

Let $A \subset M, n>0$, and $X$ be an $A$-definable subset of $M^{n}$ defined by the formula $\phi(\bar{x}, \bar{a})$, say. We can define $\operatorname{rk}(X)$ to be the largest $r$ such that in some elementary extension $N$ of $M$, there is $\bar{b} \in N^{n}$ satisfying $N=\phi(\bar{b}, \bar{a})$ and $\operatorname{Dim}\left(\left\{b_{1}, \ldots, b_{n}\right\} / A\right)$ $=r$. It is an easy exercise to show that $\operatorname{rk}(X)$ does not depend on the choice of $A$, $\bar{a}$, and $\phi$. We prove the following theorem, which has analogues for o-minimal and $C$-minimal structures (see Proposition 6.3 of [12] for the latter).

Theorem 4.12. Let $\mathfrak{M}$ be a model of a weakly o-minimal theory such that algebraic closure has the exchange property in all models of the theory. Then for all $n>0$ and all definable $X \subseteq M^{n}$, we have $\operatorname{dim}(X)=\operatorname{rk}(X)$. 
Proof. Let $X$ be $\left(a_{1}, \ldots, a_{t}\right)$-definable and put $A:=\left\{a_{1}, \ldots, a_{t}\right\}$. We may suppose that $M$ is $\omega$-saturated, since dim does not depend on the choice of model and rk is defined for $\operatorname{Th}(M, m)_{m \in M}$. Let $r:=\operatorname{dim}(X)$ and $s:=\operatorname{rk}(X)$.

We first verify that $r \leq s$. By Theorem 4.11, we may suppose that $X$ has a projection onto an open subset of $M^{r}$, and it is easy to see that this open set contains some $\bar{b}=\left(b_{1}, \ldots, b_{r}\right)$ satisfying $\operatorname{Dim}\left(\left\{b_{1}, \ldots, b_{r}\right\} / A\right)=r$. Hence $r \leq s$.

We now show that $r \geq s$. By Theorem 4.11 there is a partition of $X$ into $n$-cells $X_{1}, \ldots, X_{p}$ such that each $X_{i}$ has a homeomorphic projection $\pi_{i}$ onto an open subset of $M^{q_{i}}$. By enlarging $A$ if necessary, we may suppose that each $X_{i}$ is $A$ definable. Let $\bar{x} \in X$ with $\operatorname{Dim}\left(\left\{x_{1}, \ldots, x_{n}\right\} / A\right)=s$, and let $i \in\{1, \ldots, p\}$ be such that $\bar{x} \in X_{i}$. Let $i_{1}, \ldots, i_{s} \in\{1, \ldots, n\}$ be such that $x_{i_{1}}, \ldots, x_{i_{s}}$ are algebraically independent over $A$. Since $r \leq s$, by the exchange property we may choose these so as to include the coordinates onto which $\pi_{i}$ projects. It is now easy to see that the possibility that $s>r$ contradicts the fact that $\pi_{i}\left\lceil X_{i}\right.$ is injective.

\section{WEAKLY O-MINIMAL GROUPS AND FIELDS.}

In this section we show that every weakly o-minimal ordered group is divisible and abelian, and every weakly o-minimal ordered field is real closed. The former is easy to prove. Our proof of the latter result is intricate, and we believe there should be a more direct argument. The corresponding results in the o-minimal context are proved very rapidly in 22$]$.

\subsection{Weakly o-minimal ordered groups. We establish that}

Theorem 5.1. Every weakly o-minimal ordered group is divisible and abelian.

For the proof we require

Lemma 5.2. Let $(G,<, \cdot, 1)$ be a weakly o-minimal ordered group, and let $H$ be a definable subgroup of $G$. Then $H$ is convex in $G$.

Proof of Lemma 5.2. If $H=\{1\}$ the lemma is trivial, so we suppose that $H \neq\{1\}$. By weak o-minimality, the subgroup $H$ is the union of finitely many maximal convex subsets. Let $X$ be the greatest of these convex components with respect to the ordering induced by $<$, and let $x \in X$ with $x>1$. Let $y \in G$ with $1<y<x$. It suffices to show that $y \in H$. We have that $x<y x<x^{2}$ and, as $X$ is the greatest component in our decomposition of $H$, that $x^{2} \in X$. Since $X$ is convex, it follows that $y x \in X$. So $y x \in H$, and hence $y \in H$.

Proof of Theorem 5.1. We first show that $G$ is abelian. For this, it suffices to show that if $g, h \in G$ with $1<h<g$, then $[g, h]=1$. This follows from the lemma, since $C_{G}(g)$ is a definable group containing 1 and $g$.

To see that $G$ is divisible, observe that if $n>0$ then $G^{n}$ is a definable subgroup of $G$ containing elements cofinal in $G$, and apply the lemma.

5.2. Weakly o-minimal ordered fields. We prove the following theorem.

Theorem 5.3. Every weakly o-minimal ordered field is real closed.

We emphasize that in this theorem it is assumed just that the particular field is weakly o-minimal, and not that all models of its theory are.

Our first goal is 
Proposition 5.4. Let $F$ be a weakly o-minimal ordered field with real closure $R$, let $\alpha \in R$, and suppose that for all $\epsilon \in F$ with $\epsilon>0$ there is $\beta \in F$ such that $|\alpha-\beta|<\epsilon$. Then $\alpha \in F$.

This proposition says essentially that a weakly o-minimal ordered field is a topologically closed subset of its real closure - note that by Lemma 5.5 below, the condition 'for all $\epsilon \in F$ ' is equivalent to 'for all $\epsilon \in R$ '. Once we have established Proposition 5.4, the proof of Theorem 5.3 consists of proving the valuation-theoretic Lemmas 5.1055 .12 needed to invoke the fact that a henselian valued field with real closed residue field and divisible value group is real closed. The proof of Proposition 5.4 uses some of the dimension theory developed in Section 4, a version of the inverse function theorem for $F$, and variations of arguments from [11].

For the remainder of this subsection $F$ denotes a fixed weakly o-minimal ordered field, and $R$ is its real closure. We denote by $\bar{X}$ an $n$-tuple $\left(X_{1}, \ldots, X_{n}\right)$ of indeterminates. The first lemma is standard, but we record it for completeness.

Lemma 5.5. For all $x \in R$ with $x>0$ there is some $y \in F$ with $0<y<x$.

Proof. The real closure $R$ of $F$ is an algebraic extension of $F$, and hence all elements of $R$ are bounded in absolute value by elements of $F$. The conclusion follows.

This lemma essentially says that the topology on $F$ given by the ordering is exactly that induced from the ordering on $R$. The corresponding statement thus holds for the topologies on $F^{n}$ and $R^{n}$ as well. The next lemma is an immediate consequence of this.

Lemma 5.6. Let $p_{1}(\bar{X}), \ldots, p_{n}(\bar{X}) \in F[\bar{X}]$ and let $\bar{p}:=\left(p_{1}, \ldots, p_{n}\right)$. Suppose that $V \subset R^{n}$ is an open box satisfying

(a) $V \cap F^{n} \neq \emptyset$,

(b) $\bar{p}^{-1} \uparrow_{V}: V \rightarrow R^{n}$ is a continuous well-defined function.

Then $\bar{p}^{-1}\left\lceil_{V \cap F^{n}}\right.$ is continuous, where the domain of $\bar{p}^{-1} \uparrow_{V \cap F^{n}}$ has the topology induced from $F^{n}$ and its range has the topology induced from $R^{n}$.

Let $K$ be an ordered field, $n>0, f_{1}, \ldots, f_{n} \in K\left[X_{1}, \ldots, X_{n}\right], \bar{f}=\left(f_{1}, \ldots, f_{n}\right)$, and $\bar{a} \in K^{n}$. By $J_{\bar{f}}(\bar{a})$ we denote the Jacobian of $\bar{f}$ at $\bar{a}$. We now require a version of the inverse function theorem suitable for $R$.

Theorem 5.7 (Inverse Function Theorem). Let $f_{1}(\bar{X}), \ldots, f_{n}(\bar{X}) \in R[\bar{X}]$, and let $\bar{f}:=\left(f_{1}, \ldots, f_{n}\right)$. Let $U^{*} \subset R^{n}$ be an open box and $V^{*}:=\bar{f}\left(U^{*}\right)$. Let $\bar{a} \in U^{*}$, and suppose $J_{\bar{f}}(\bar{a}) \neq 0$. Then there are an open box $U \subseteq U^{*}$, an open set $V \subseteq V^{*}$, and a unique function $g$ definable in $R$ such that

(i) $\bar{a} \in U$ and $\bar{f}(\bar{a}) \in V$,

(ii) $\bar{f}^{-1}(V)=U$,

(iii) $\bar{f} \Gamma_{U}$ is injective,

(iv) $g$ is defined on $V, g(V)=U$, and $g(\bar{f}(\bar{x}))=\bar{x}$ for all $\bar{x} \in U$,

(v) $g$ is continuous and differentiable on $V$.

Proof. Since we have required that $U$ and $U^{*}$ be open boxes, for given degrees of the polynomials $f_{i}, i \leq n$, the statements in the theorem are first-order. The theorem as we have stated it holds if $R=\mathbb{R}$ - see for example [1] — and hence holds for any real closed field. 
By an open $R$-box (respectively $F$-box) we mean an open box in $R^{n}$ (respectively $F^{n}$ ) which is the cartesian product of open intervals whose endpoints lie in $R$ (respectively $F$ ). Combining the last two facts, we obtain

Corollary 5.8. Let $p_{1}(\bar{X}), \ldots, p_{n}(\bar{X}) \in F[\bar{X}]$ and $\bar{p}=\left(p_{1}, \ldots, p_{n}\right)$. Also, let $B$ be an open box in $R^{n}$ which is the cartesian product of open intervals whose endpoints all lie in $F$. Suppose for some $\bar{a} \in B \cap F^{n}$ that $J_{\bar{p}}(\bar{a}) \neq 0$. Then there is an open $R$-box $U \subset B$ with $\bar{a} \in U$ such that $\bar{p} \uparrow_{U}$ is injective, $V:=\bar{p}(U)$ open, and $\bar{p}^{-1} \uparrow_{V \cap F^{n}}$ is continuous, where the domain of $\bar{p}^{-1} \uparrow_{V \cap F^{n}}$ has the topology induced from $F$ and the range has the $R$-topology.

Proof. The existence of $U$ and $V$ follows from Theorem [5.7] and the continuity of $\bar{p}^{-1} \uparrow_{V \cap F^{n}}$ follows from Lemma 5.6 .

We are now ready to prove a version of the inverse function theorem for $F$. Essentially, we are proving a version of Theorem 4.10 for polynomial functionsrecall that in Theorem 4.10 the hypotheses include the provision that the theory be weakly o-minimal. The main point of this result is that the set $U$ is an $F$-box and not just an $R$-box.

Proposition 5.9. Let $p_{1}(\bar{X}), \ldots, p_{n}(\bar{X}) \in F[\bar{X}]$ and $\bar{p}:=\left(p_{1}, \ldots, p_{n}\right)$, and let $\bar{a} \in F^{n}$ with $J_{\bar{p}}(\bar{a}) \neq 0$. Then for every open $F$-box $U$ containing $\bar{a}$, the set $\bar{p}(U)$ has non-empty interior in the topology on $F^{n}$.

Proof. We use induction on $n$. The case $n=1$ is immediate since $F$ is weakly o-minimal. So we assume the result for $n-1$, and let $\bar{p}, \bar{a}$, and $U=I_{1} \times \cdots \times I_{n}$ be as in the statement of the proposition. Since $J_{\bar{p}}(\bar{a}) \neq 0$, some $(n-1) \times(n-1)$ minor of the matrix $\left.\left(\partial p_{i} / \partial x_{j}\right)\right|_{\bar{a}}$ has non-zero determinant. For ease of notation we suppose that this minor consists of the first $n-1$ rows and first $n-1$ columns.

Now consider the function $\bar{p}^{*}: F^{n-1} \longrightarrow F^{n-1}$ defined by

$$
\bar{p}^{*}\left(X_{1}, \ldots, X_{n-1}\right):=\left(p_{1}\left(X_{1}, \ldots, X_{n-1}, a_{n}\right), \ldots, p_{n-1}\left(X_{1}, \ldots, X_{n-1}, a_{n}\right)\right) .
$$

Also put $U^{*}:=U \cap\left(F^{n-1} \times\left\{a_{n}\right\}\right)$, and let $\pi: F^{n} \longrightarrow F^{n-1}$ denote projection onto the first $n-1$ coordinates. Then $J_{\bar{p}^{*}}(\pi(\bar{a})) \neq 0$. By Corollary 5.8 there is an $R$-box $W \subset \pi\left(U^{*}\right)$ containing $\pi(\bar{a})$ and satisfying the conclusion of the corollary for $p^{*}$. Applying Lemma 5.5 we find an $F$-box $W^{\prime} \subset W$ also containing $\pi(\bar{a})$. The induction hypothesis tells us that for every open $F$-box $B \subset \pi\left(U^{*}\right)$ containing $\pi(\bar{a})$ we have that $\bar{p}^{*}(B)$ has non-empty interior in $F^{n-1}$. We now find an $F$-box $U_{0} \subset W^{\prime}$ such that $\bar{p}^{*}\left(U_{0}\right)$ is open. Invoking the conclusion of Corollary $5.8-$ which holds for $W$ and hence for $U_{0}$-we see that $\bar{p}^{*}$ induces a homeomorphism between $U_{0} \subset \pi\left(U^{*}\right)$ and an open subset $V_{0} \subset F^{n-1}$, where both $U_{0}$ and $V_{0}$ carry the topology induced from $F^{n-1}$.

Notice that $U_{0} \times I_{n} \subset F^{n}$ is an open $F$-box. Also, for each $\bar{x} \in U_{0}$, let $\bar{x}^{*}:=$ $\left(\bar{x}, a_{n}\right)$. For each $\bar{x} \in U_{0}$, we colour $\bar{x}$ with just one of the three colours, red, green, or blue, as follows:

i. Colour $\bar{x}$ red if there is some $z<p_{n}\left(\bar{x}^{*}\right)$ (which we may take in $F$ ) such that

$$
\left\{\left(p_{1}\left(\bar{x}^{*}\right), \ldots, p_{n-1}\left(\bar{x}^{*}\right)\right)\right\} \times\left\{w \in F: z<w<p_{n}\left(\bar{x}^{*}\right)\right\} \subset \bar{p}\left(U_{0} \times I_{n}\right) .
$$

ii. Colour $\bar{x}$ green if $\bar{x}$ is not coloured red and there is some $z>p_{n}\left(\bar{x}^{*}\right)$ (in $F$ ) such that

$$
\left\{\left(p_{1}\left(\bar{x}^{*}\right), \ldots, p_{n-1}\left(\bar{x}^{*}\right)\right)\right\} \times\left\{w \in F: p_{n}\left(\bar{x}^{*}\right)<w<z\right\} \subset \bar{p}\left(U_{0} \times I_{n}\right) .
$$


iii. Colour $\bar{x}$ blue if it is coloured neither red nor green.

By Theorem 4.2, after replacing $U_{0}$ by a subset if necessary, we may suppose that all points of $U_{0}$ are red, or all are green, or all are blue. Define $h: V_{0} \longrightarrow F$ by putting

$$
h(\bar{y})=p_{n}\left(\left(\left(\bar{p}^{*}\right)^{-1}(\bar{y})\right)^{*}\right)
$$

for each $\bar{y} \in V_{0}$. As $h$ is a composition of continuous mappings, it is continuous. The remainder of the argument divides into three cases.

Case 1. All points of $U_{0}$ are blue. We show that this leads to a contradiction. By weak o-minimality we define functions $h_{1}, h_{2}: V_{0} \longrightarrow \bar{F}$, where the ranges are sorts in $\bar{F}$, as follows. Let $\bar{y} \in V_{0}$ with $\bar{y}=\bar{p}^{*}(\bar{x})$. We let $h_{1}(\bar{y})$ and $h_{2}(\bar{y})$ be such that $h_{1}(\bar{y})<p_{n}\left(\bar{x}^{*}\right)<h_{2}(\bar{y})$ and $\left(h_{1}(\bar{y}), h_{2}(\bar{y})\right)$ is the maximal convex set $C \subset F$ which contains $p_{n}\left(\bar{x}^{*}\right)$ in its interior and satisfies

$$
\bar{p}\left(U_{0} \times I_{n}\right) \cap\left[\left\{\left(p_{1}\left(\bar{x}^{*}\right), \ldots, p_{n-1}\left(\bar{x}^{*}\right)\right)\right\} \times C\right]=\left\{\bar{p}\left(\bar{x}^{*}\right)\right\} .
$$

By Theorem 4.3 and the continuity of $h: V_{0} \longrightarrow F$, there is an open box

$$
W \subset\left\{\left(p_{1}\left(\bar{x}^{*}\right), \ldots, p_{n-1}\left(\bar{x}^{*}\right), w\right): \bar{x} \in U_{0}, h_{1}\left(\bar{p}^{*}(\bar{x})\right)<w<h_{2}\left(\bar{p}^{*}(\bar{x})\right)\right\} .
$$

By continuity of $\bar{p}$, it follows that $\bar{p}^{-1}(W) \cap F^{n}$ is open in $F^{n}$. However, as $\bar{p}^{-1}(W) \cap\left(U_{0} \times I_{n}\right) \subset U_{0} \times\left\{a_{n}\right\}$ and is non-empty, it is not open in $F^{n}$. This is a contradiction.

Case 2. All points of $U_{0}$ are red. In this case there is a definable function $g$ : $V_{0} \longrightarrow \bar{F}$ such that for each $\bar{y} \in V_{0}$, if $\bar{y}:=\bar{p}^{*}(\bar{x})$, where $\bar{x} \in U_{0}$, then $g(\bar{y})$ is the infimum of

$$
\left\{z \in F: \forall w\left[z<w<p_{n}\left(\bar{x}^{*}\right)\right] \longrightarrow\left[\left(p_{1}\left(\bar{x}^{*}\right), \ldots, p_{n-1}\left(\bar{x}^{*}\right), w\right) \in \bar{p}\left(U_{0} \times I_{n}\right)\right]\right\} .
$$

Since $h$ is continuous and $g(\bar{y})<h(\bar{y})$ for all $\bar{y} \in V_{0}$, by Theorem 4.3 there is an open box $W \subset F^{n}$ such that for all $\bar{z}$ in $W$, there is some $\bar{x} \in U_{0}$ such that $z_{i}=p_{i}\left(\bar{x}^{*}\right)$ for $i=1, \ldots, n-1$ and

$$
g\left(\left(z_{1}, \ldots, z_{n-1}\right)\right)<z_{n}<p_{n}\left(\bar{x}^{*}\right) .
$$

Then $W \subseteq \bar{p}(U)$, as required.

Case 3. All points of $U^{\prime}$ are green. The argument is as in Case 2.

This completes the proof of Proposition 5.9.

Now we are prepared to prove Proposition 5.4

Proof of Proposition 5.4. We adapt a technique from [11. Suppose that the proposition is false for $\alpha$, and let $\alpha_{1}=\alpha, \alpha_{2}, \ldots, \alpha_{n}$ be the distinct conjugates of $\alpha$ over $F$. For this proof let $\bar{X}:=\left(X_{1}, \ldots, X_{n-1}\right)$. Put

$$
g(\bar{X}, Y)=\prod_{i=1}^{n}\left(Y-\sum_{j=0}^{n-1} X_{j} \alpha_{i}^{j}\right)=\sum_{j=0}^{n-1} G_{j}(\bar{X}) Y^{j}+Y^{n} .
$$

We now assert that:

(1) $G_{j}(\bar{X}) \in F[\bar{X}]$ for $j=1, \ldots, n-1$.

(2) If $x_{0}, \ldots, x_{n-1}$ are algebraically independent over $F$, then so are $G_{0}(\bar{x}), \ldots$, $G_{n-1}(\bar{x})$.

(3) If $\bigvee_{j=1}^{n-1} x_{j} \neq 0$, then $g(\bar{x}, Y)$ has no roots in $F$. 
Statement (1) is immediate since each $G_{i}$ is symmetric in $\alpha_{1}, \ldots, \alpha_{n}$. For (2), we refer the reader to the argument in the proof of Theorem 1 in [11]. Assertion (3) holds because $\sum_{j=0}^{n-1} x_{j} \alpha_{i}^{j} \notin F$ for each $i=1, \ldots, n$.

We define $\bar{G}: F^{n} \longrightarrow F^{n}$ by

$$
\bar{G}(\bar{x}):=\left(G_{0}(\bar{x}), \ldots, G_{n-1}(\bar{x})\right) .
$$

Let $J_{\bar{G}}(\bar{X})$ be the Jacobian $\operatorname{det}\left(\partial G_{i} / \partial X_{j}\right)$. Now we claim that

(4) there is some $\bar{d} \in F^{n}$ such that $J_{\bar{G}}(\bar{d}) \neq 0$ and $\bigvee_{j=1}^{n-1} d_{j} \neq 0$.

To establish (4) we first choose an elementary extension $K$ of $F$, with $b_{0}, \ldots, b_{n-1} \in$ $K$ algebraically independent over $F$. By $(2)$, we have that $G_{0}(\bar{b}), \ldots, G_{n-1}(\bar{b})$ are algebraically independent over $F$, and so $J_{\bar{G}}(\bar{b}) \neq 0$. Furthermore, $\bigvee_{j=1}^{n-1} b_{j} \neq 0$. Hence

$$
K \models \exists \bar{x}\left(J_{\bar{G}}(\bar{x}) \neq 0 \wedge \bigvee_{j=1}^{n-1} x_{j} \neq 0\right),
$$

whence

$$
F \models \exists \bar{x}\left(J_{\bar{G}}(\bar{x}) \neq 0 \wedge \bigvee_{j=1}^{n-1} x_{j} \neq 0\right) .
$$

So we may choose some $\bar{d} \in F^{n}$ to be a witness in $F$ for this formula, which proves (4).

By Corollary 5.8 and Lemma 5.5, there is an open set $W \subset F^{n}$ containing $\bar{d}$ such that $\bar{G} \uparrow_{W}$ is injective, continuous, and has continuous inverse (into $F^{n}$ ). Proposition 5.9 tells us that there is an open $U \subset W$ with $\bar{d} \in U$ such that $\bar{G}(U)$ has non-empty interior. By choosing $U$ within a sufficiently small neighbourhood of $\bar{d}$, we may suppose that $J_{\bar{G}}(\bar{x}) \neq 0 \wedge \bigvee_{j=1}^{n-1} x_{j} \neq 0$ for all $\bar{x} \in U$. Now let $B$ be an open $F$-box contained in $\bar{G}(U)$. By replacing $\bar{d}$ by an appropriate element of $U$ if necessary, we may suppose that $\bar{e}:=\bar{G}(\bar{d}) \in B$.

For $y \neq 0$ we now define the continuous function

$$
f(y):=-\left(y^{n}+\sum_{j=0}^{n-2} e_{j} y^{j}\right) / y^{n-1} .
$$

For every $\gamma \neq 0$ we have

$$
\gamma^{n}+f(\gamma) \gamma^{n-1}+\sum_{j=0}^{n-2} e_{j} \gamma^{j}=0
$$

Observe that

$$
\sum_{j=0}^{n-1} d_{j} \beta^{j} \rightarrow \sum_{j=0}^{n-1} d_{j} \alpha^{j} \neq 0 \quad \text { as } \beta \rightarrow \alpha .
$$

Since $\sum_{j=0}^{n-1} d_{j} \alpha^{j}$ is a zero of $g(\bar{d}, Y)$, we also have

$$
\sum_{i=0}^{n-1} e_{i}\left(\sum_{j=0}^{n-1} d_{j} \alpha^{j}\right)^{i}+\left(\sum_{j=0}^{n-1} d_{j} \alpha^{j}\right)^{n}=0
$$


By putting $\gamma=\sum_{j=0}^{n-1} d_{j} \alpha^{j}$ in (5) and applying (6) and (7) we obtain, in $R$,

$$
f\left(\sum_{j=0}^{n-1} d_{j} \beta^{j}\right) \rightarrow e_{n-1} \quad \text { as } \beta \rightarrow \alpha .
$$

From the hypothesis of the proposition and Lemma 5.5 there is some $\beta \in F$ such that

$$
\sum_{j=0}^{n-1} d_{j} \beta^{j} \neq 0 \text { and }\left(e_{0}, \ldots, e_{n-2}, f\left(\sum_{j=0}^{n-1} d_{j} \beta^{j}\right)\right) \in B \subset \bar{G}(U) .
$$

Hence there is some $\bar{c} \in U$ such that

$$
\bar{G}(\bar{c})=\left(e_{0}, \ldots, e_{n-2}, f\left(\sum_{j=0}^{n-1} d_{j} \beta^{j}\right)\right) .
$$

By our choice of $U$ we have that $\bigvee_{j=1}^{n-1} c_{j} \neq 0$. It then follows from (3) that

$$
Y^{n}+f\left(\sum_{j=0}^{n-1} d_{j} \beta^{j}\right) Y^{n-1}+\sum_{j=0}^{n-2} e_{j} Y^{j}
$$

has no zero in $F$. However we see from (5) that $\sum_{j=0}^{n-1} d_{j} \beta^{j}$ is a zero in $F$ of this polynomial. This is a contradiction, and the proof of Proposition 5.4 is complete.

We now turn to the final valuation-theoretic arguments for the proof of Theorem 5.3. Recall that we have fixed $F$ to be a weakly o-minimal ordered field with real closure $R$. Let $v: F \longrightarrow \Gamma$ be the archimedean valuation, where $\Gamma$ denotes an ordered abelian group. If $\Gamma=\{0\}$, then $F$ is dense in $\mathbb{R}$. Hence by Proposition 5.4 $F=\mathbb{R}$, and the theorem is proved. So we may assume that $\Gamma \neq\{0\}$. Let $V$ be the valuation ring of $v$, let $M$ be its maximal ideal, and let $\bar{F}$ denote the residue field. We now require three final lemmas to complete the proof of Theorem 5.3 .

Lemma 5.10. The value group $\Gamma$ is divisible.

Proof. Since $\left(F^{>0}, \cdot,<\right)$ is a weakly o-minimal group, by Theorem 5.1 we observe that

$$
(\forall x \in F)\left[x>0 \Longrightarrow(\exists y \in F) y=x^{n}\right],
$$

and so $\Gamma$ is divisible.

Lemma 5.11. The residue field $\bar{F}$ is real closed.

Proof. For a contradiction, suppose that $\bar{F}$ is not real closed. We claim that

$$
v \text { is definable. }
$$

To prove this, let $p(X) \in F[X]$ be such that the residue $\bar{p}$ changes sign on $\bar{F}$ but $\bar{p}$ has no root in $\bar{F}$ - such a polynomial exists as $\bar{F}$ is assumed not to be real closed.

Without loss of generality we identify the prime subfield of $F$ with $\mathbb{Q}$. Since $v$ is the archimedean valuation, the set of classes of elements of $\mathbb{Q}$ under $v$ is dense in $\bar{F}$. By the continuity of $\bar{p}$ we see that for all positive integers $n$ there is some $q \in \mathbb{Q}$ such that $|p(q)|<1 / n$. Since $\bar{p}$ has no root in $\bar{F}$, we also have for all $x \in F$ that there is some positive integer $m$ satisfying $|p(x)|>1 / m$. We now set

$$
M^{*}:=\{\epsilon \in F:(\forall x \in F)|p(x)|>|\epsilon|\} .
$$


By the preceding observations, $M^{*}$ is the set of infinitesimals of $F$; that is, $M^{*}=M$. So $M$ is definable, and hence the valuation $v$ is definable also.

We thus have that $\bar{F}$ is interpretable in $F$ as a set of equivalence classes modulo $M$. We next observe that $\bar{F}$ itself carries the structure of a weakly o-minimal ordered field; that is, an $\bar{F}$-definable subset of $\bar{F}$ is a finite union of convex subsets of $\bar{F}$. This is almost immediate. If $X \subseteq \bar{F}$ is $\bar{F}$-definable, then $X$ lifts to the definable subset $X^{*}:=\{x \in V: x+M \in X\}$ of $F$. As $X^{*}$ is the union of finitely many convex subsets of $F$, it follows that $X$ too is a finite union of convex subsets of $\bar{F}$.

Since $\bar{F}$ is dense in its real closure, we may apply Proposition 5.4 to $\bar{F}$ to conclude that $\bar{F}$ is real closed.

Lemma 5.12. The field $F$ is henselian.

Proof. For a contradiction, we suppose that $F$ is not henselian. By (5) of Theorem 4 (p. 186) of [24], it is easy to see that there is a polynomial

$$
p(X):=X^{n}+a X^{n-1}+\sum_{j=0}^{n-2} c_{j} X^{j} \in V[X]
$$

of degree $n \geq 2$ with $v(a)=0$ and $v\left(c_{i}\right)>0$ for $i=0, \ldots, n-2$ which has no root in $F$. Since $R$, the real closure of $F$, is henselian - again, with respect to the archimedean valuation - we additionally see by Theorem 4(5) of [24] that there is some $\alpha \in R$ such that $p(\alpha)=0$ and $v(a-\alpha)>0$. Notice as well that, as $\bar{\alpha}$ is a simple root of $\bar{p}(X)$, the residue of $p(X)$ over $\bar{F}$, we have that $v\left(p^{\prime}(\alpha)\right)=0$.

We now define

$$
S:=\{g \in \Gamma: g>0 \wedge(\exists \beta \in F) v(\beta-\alpha)=g\} .
$$

As $v(a-\alpha)>0$, it follows that $S \neq \emptyset$. Let $\Delta$ be the convex subgroup of $\Gamma$ generated by $S$. We assert that

Claim 5.12.1. The set $S$ is cofinal in $\Delta$.

Proof of Claim. It suffices to prove that if $g \in S$ then there is some $\gamma \in F$ so that $v(\gamma-\alpha) \geq 2 g$. So let $g \in S$ be given and let $\beta \in F$ be such that $v(\beta-\alpha)=g$. It is easy to see that $v\left(p^{\prime}(\beta)\right)=v\left(p^{\prime}(\alpha)\right)$-using Taylor's theorem around $\alpha$, for example - and hence that $v\left(p^{\prime}(\beta)\right)=0$. In particular, $p^{\prime}(\beta) \neq 0$, and so we may set $\gamma:=\beta-p(\beta) / p^{\prime}(\beta) \in F$. By Taylor's theorem we have

$$
\gamma-\alpha=\frac{p^{\prime \prime}(\beta)}{2 ! p^{\prime}(\beta)}(\alpha-\beta)^{2}+\cdots+\frac{p^{(n)}(\beta)}{n ! p^{\prime}(\beta)}(\alpha-\beta)^{n} .
$$

This, combined with the fact that $v\left(p^{\prime}(\beta)\right)=0$, yields that $v(\gamma-\alpha) \geq 2 g$, as required.

Let

$$
I:=\{x \in F: v(x)>\Delta\}
$$

We next assert that

Claim 5.12.2. The set $I$ is definable in F. 
Proof of Claim. We first show that if $v(\beta-\alpha)=g>0$, then $v(p(\beta))=v(\beta-\alpha)=g$. To see this we first use Taylor's theorem again to write

$$
p(\beta)=p^{\prime}(\alpha)(\beta-\alpha)+\frac{p^{\prime \prime}(\alpha)}{2 !}(\beta-\alpha)^{2}+\cdots+\frac{p^{(n)}(\alpha)}{n !}(\beta-\alpha)^{n} .
$$

Since $\bar{\alpha}$ is a simple root of $\bar{p}(X)$, the residue of $p(X)$ over $\bar{F}$, we have that $v\left(p^{\prime}(\alpha)\right)=$ 0 , and our assertion follows.

Now let $J$ be an interval of $F$ such that $\alpha$ is in the convex closure of $J$ in $R$ and $v(\beta-\alpha)>0$ for all $\beta \in J$. To see that such an interval $J$ exists, we use the result of the preceding paragraph with $\beta=a$ to obtain that $v(p(a))=v(a-\alpha)>0$. Then, as $p(a) \in F$ and by Lemma [5.10] we have that $\Gamma$ is divisible, there is some $c \in F$ with $v(c-a)=v(a-\alpha) / 2$, and the assertion follows. By Claim 5.12.1 and what we proved in the preceding paragraph,

$$
I=\{\epsilon:(\forall x \in J)|p(x)|>|\epsilon|\},
$$

and hence is definable in $F$.

Now we complete the proof of the lemma. By Claim 5.12.2 we see that the natural valuation $v_{0}: F \longrightarrow \Gamma / \Delta$ is definable. Let $\bar{F}_{0}$ be its residue field. As in the proof of Lemma 5.11, we have that $\bar{F}_{0}$ carries the structure of a weakly o-minimal ordered field. By the choice of $\Delta$, there are arbitrarily good approximations in $\bar{F}_{0}$ to $\bar{\alpha}$, the residue of $\alpha$ in $\bar{F}_{0}$, and hence by Proposition 5.4 we must have that $\bar{\alpha} \in \bar{F}_{0}$. Finally, let $J$ be an interval in $F$ as in the proof of Claim 5.12.2. that is, $\alpha$ lies in the convex closure of $J$ in $R$ and for all $c \in J$ we have $v(c-\alpha)>0$. As $v(p(\beta))=v(\beta-\alpha)$ for all $\beta \in J$, it follows by our choice of $\Delta$ that $\bar{p}(X)$, the residue of $p(X)$ over $\bar{F}_{0}$, has no root in $\bar{J}$, the residue of $J$ in $\bar{F}_{0}$. But since $\bar{\alpha} \in \bar{J}$, we reach a contradiction.

We are finally ready to prove the principal result of this section.

Proof of Theorem 5.3] By Lemmas [5.10 5.12] the theorem follows immediately from [18] (see [23]).

We conclude this section with:

Corollary 5.13. A weakly o-minimal ordered unitary commutative ring $R$ is a real closed ring.

Proof. If $R$ is a field then we are done, and so we suppose not. We then note that if $a, b \in R$ with $0<b \leq a$, then $a \mid b$. To see this, observe by Lemma 5.2 that $a R$ is a convex subgroup of $(R,+)$ and $a \in a R$. In particular, for every $a>1$ we have $1 \in a R$. It follows that $\{a \in R: a>0 \wedge(\exists y) a y=1\}$ is a cofinal and-by Lemma 5.2 convex subgroup of the semigroup $\left(R^{>0}, \cdot\right)$. It is routine to verify that the set of non-units in $R$ forms an ideal. Hence $R$ is an ordered valuation ring whose fraction field $F$-obtained by adding just inverses of elements in the maximal ideal $M$-we interpret in the obvious way in $R$. It follows easily that $F$ is weakly o-minimal. By Theorem 5.3 we have that $F$ is real closed, and so by $[5]$ we conclude that $R$ is real closed also. 


\section{A DICHOTOMY FOR WEAKLY O-MINIMAL EXPANSIONS OF FIELDS}

Throughout this section, $\mathfrak{M}=(M,<,+,-, \cdot, 0,1, \ldots)$ is a weakly o-minimal expansion of an ordered field. We assume only that $\mathfrak{M}$ is weakly o-minimal, not that all models of its theory are.

We first establish in Section 6.1 a model-theoretic dichotomy for such structures $\mathfrak{M}$ which corresponds to whether or not a non-trivial convex valuation is definable in $\mathfrak{M}$. In Section 6.2 we show - subject to the proof of the main technical proposition, Lemma 6.10 that a weakly o-minimal expansion of an ordered field having no nontrivial definable convex valuation enjoys many of the good properties of o-minimal structures. These include algebraic boundedness (Theorem 6.7) and cell decomposition in which the cells have continuous boundary functions (Theorem 6.6). In Section 6.3 we provide the deferred proof of Lemma 6.10 .

6.1. Valuational and non-valuational structures. A cut in $M$ is a pair $\left(C_{1}, C_{2}\right)$ of subsets of $M$ such that $C_{1}<C_{2}$ and $M=C_{1} \cup C_{2}$.

Definition 6.1. The cut $\left(C_{1}, C_{2}\right)$ is valuational if

$$
\exists \epsilon>0 \forall x \in C_{1}\left(\forall y \in C_{2}\right)(y-x>\epsilon) .
$$

The structure $\mathfrak{M}$ is of valuational type if it has a definable valuational cut; it is of non-valuational type otherwise.

Observe that Definition 6.1 makes sense even if $\mathfrak{M}$ is just an expansion of an ordered group; in what follows we are, however, interested almost exclusively in the case that $\mathfrak{M}$ is an expansion of an ordered field. We now move toward establishing the result, Theorem 6.3 that justifies this terminology. We first require a lemma, as well as some additional notation and terminology.

Lemma 6.2. Let $\mathfrak{M}$ be a weakly o-minimal expansion of an ordered field, and suppose that $M$ has a proper non-empty convex definable additive subgroup $I$. Then $\mathfrak{M}$ has a non-trivial definable convex valuation; that is, there is a non-trivial definable convex valuation ring $R \subset M$.

Proof. We define

$$
R:=\{x \in M: x I \subseteq I\}
$$

We assert that

(a) $1 \in R$,

(b) $R$ is convex,

(c) if $x, y \in R$ then $x y \in R$,

(d) if $x, y \in R$ then $x+y \in R$,

(e) $R \neq M$,

(f) if $x \in M \backslash\{0\}$ then at least one of $x$ or $x^{-1}$ is contained in $R$.

It follows from (a)-(f) that $R$ is a non-trivial proper definable convex valuation ring in $M$, as required.

Statements (a), (b), and (c) are immediate, and (d) follows from the fact that $I$ is additively closed. If (e) were false then $I$ would be a proper non-trivial ideal in $M$, which is impossible. Finally, for (f) observe that if $x \in M \backslash\{0\}$ then either $|x| \leq 1$ or $\left|x^{-1}\right| \leq 1$; hence (f) follows from (a) and (b). 
Let $\bar{M}$ denote the Dedekind completion of $M$ as an ordered set. For $a, b \in M$ with $a \leq b$, set $(a, b)_{\bar{M}}:=\{z \in \bar{M}: a<z<b\}$. Now let $A \subseteq \bar{M}$. We define the equivalence relation $E_{A}$ on $M$ by putting

$$
\begin{aligned}
x E_{A} y & \text { if and only if } \quad \begin{array}{l}
x \\
\text { or }
\end{array} \quad \begin{array}{l}
\text { or } \\
y
\end{array} \leq x \text { and }(y, x)_{\bar{M}} \cap A=\emptyset \\
& =\emptyset .
\end{aligned}
$$

Such a set $A \subseteq \bar{M}$ is said to be a discrete sort if

(i) $A$ is a sort in $\bar{M}$ in the sense of Section 1.2 , and

(ii) the equivalence relation $E_{A}$ contains infinitely many infinite classes.

Now we state and prove the result that justifies Definition 6.1

Theorem 6.3. Let $\mathfrak{M}$ be a weakly o-minimal expansion of an ordered field. Then the following are equivalent:

(i) $\mathfrak{M}$ is valuational;

(ii) $\mathfrak{M}$ has a non-trivial definable convex valuation, that is, there is a non-trivial definable convex valuation ring $R \subset M$;

(iii) $\mathfrak{M}$ has a discrete sort.

Proof. We first note that (ii) evidently implies (iii). For if $R \subset M$ is a non-trivial definable convex valuation ring, we see that

$$
A:=\{\sup (x+R): x \in M\}
$$

is a discrete sort. It also is easy to see that (ii) implies (i). For let $R$ be as above, and let $J$ denote the maximal ideal of $R$ consisting of its non-invertible elements. Let $\epsilon \in J$ with $\epsilon>0$ and let

$$
C_{1}:=\{x: x<0\} \cup\{x: x \in J\} \quad \text { and } \quad C_{2}:=M \backslash C_{1} .
$$

As $C_{1}$ and $C_{2}$ are definable, and for $a \in C_{1}$ and $b \in C_{2}$ we have $b-a>\epsilon$, we finish.

Now we prove that (i) $\rightarrow$ (ii). Let $\left(C_{1}, C_{2}\right)$ be a definable valuational cut in $M$. Then there is $\epsilon>0$ so that for all $a \in C_{1}$ and $b \in C_{2}$ we have $b-a>\epsilon$. Define

$$
D:=\left\{\delta>0:\left(\forall x \in C_{1}\right)\left(\forall y \in C_{2}\right) y-x>\delta\right\} .
$$

We now assert that $D$ is additively closed. For this let $\delta_{1}, \delta_{2} \in D$, and let $u \in C_{1}$ and $v \in C_{2}$. Since $\delta_{1} \in D$ and $u \in C_{1}, u+\delta_{1} \in C_{1}$. As $\delta_{2} \in D$, it follows that $v-\left(u+\delta_{1}\right)>\delta_{2}$. Hence $v-u>\delta_{1}+\delta_{2}$, and so, as $u$ and $v$ were chosen arbitrarily, we have $\delta_{1}+\delta_{2} \in D$, as required. We thus see that

$$
I:=\bigcup\{(-x, x): x \in D\}
$$

is a non-trivial convex definable additive subgroup of $M$. Furthermore, $I \neq M$, since for every $c_{1} \in C_{1}$ and $c_{2} \in C_{2}$ we have that $c_{2}-c_{1} \notin I$. By Lemma 6.2 we conclude that $M$ has a non-trivial definable convex valuation, as required.

It remains to prove that (iii) $\rightarrow$ (ii). Let $A$ be a discrete sort in $\mathfrak{M}$. So there is a definable equivalence relation $E_{A}$ on $M$ with infinitely many infinite classes, each of which is a convex set. For every $E$-class $B$, we define

$$
\begin{aligned}
& I_{B}:=\left\{b_{1}-b_{2}: b_{1}, b_{2} \in B\right\}, \\
& J_{B}:=\left\{b \in I_{B}: b+b \in I_{B}\right\}, \\
& B_{1}:=\left\{b \in B: \forall x \in B\left(x<b \rightarrow b-x \in J_{B}\right)\right\}, \\
& B_{2}:=\left\{b \in B: \forall x \in B\left(x>b \rightarrow x-b \in J_{B}\right)\right\} .
\end{aligned}
$$


First observe that $I_{B}$ is convex and symmetric about 0 , that $B_{1}$ is an initial segment of $B$, and that $B_{2}$ is a final segment of $B$. Also, $J_{B} \neq\{0\}$ since $(M,+)$ is divisible. We now show that $J_{B}=I_{B}$ for some $E_{A}$-class $B$. This suffices, for if $I_{B}=J_{B}$ for an $E_{A}$-class $B$, then $I_{B}$ is a non-trivial additive subgroup of $M$ that clearly is not equal to $M$, and thus (ii) follows from Lemma 6.2.

So for a contradiction suppose that we have the proper inclusions

$$
\{0\} \subset J_{B} \subset I_{B} \text { for each } E_{A} \text {-class } B \text {. }
$$

Then, for every $E_{A}$-class $B$ there are $b_{1}, b_{2} \in B$ such that $b_{1}<b_{2}$ and $b_{2}-b_{1}>J_{B}$. Hence $b_{2} \in B \backslash B_{1}$ and so $B_{1} \neq B$. Similarly, we have $B_{2} \neq B$ for every $E_{A}$-class $B$. Since

$$
\bigcup\left\{B_{1}: B \text { is an } E_{A^{-}} \text {-class }\right\} \text { and } \bigcup\left\{B_{2}: B \text { is an } E_{A^{-c l a s s}}\right\}
$$

are definable, and there are infinitely many $E_{A}$-classes, it follows by weak ominimality that there is an $E_{A}$-class $B$ such that $B_{1}=B_{2}=\emptyset$. For such an $E_{A}$-class $B$ let $b \in B$. As $b \notin B_{1}$, there is some $a_{1} \in B$ such that $a_{1}<b$ and $b-a_{1}>J_{B}$. Also, since $b \notin B_{2}$, there is some $a_{2} \in B$ such that $b<a_{2}$ and $a_{2}-b>J_{B}$. It follows that

$$
\left(b-a_{1}\right)+\left(a_{2}-b\right) \geq 2 \cdot \operatorname{Min}\left\{b-a_{1}, a_{2}-b\right\}>I_{B} .
$$

Hence $a_{2}-a_{1}>I_{B}$, contradicting that $a_{1}, a_{2} \in B$.

6.2. Model-theoretic and topological properties of non-valuational structures. In this subsection we show that a weakly o-minimal expansion of an ordered field which is of non-valuational type is very close to an o-minimal structure. In particular, such a structure has a good cell decomposition, and every model of the theory of such a structure is weakly o-minimal. We first show that Theorem 3.4 can be improved for a weakly o-minimal expansion of a field of non-valuational type.

Proposition 6.4. Suppose that $\mathfrak{M}$ is a weakly o-minimal expansion of a field of non-valuational type. Let $A \subset \bar{M}$ be a sort, and let $f: M \longrightarrow M \cup A$ be a definable function. Then $\operatorname{dom}(f)$ can be partitioned as

$$
\operatorname{dom}(f)=X \cup I_{1} \cup \ldots \cup I_{m},
$$

where $X$ is finite, and for each $j=1, \ldots, m$, the set $I_{j}$ is a definable convex set and $f\left\lceil_{I_{j}}\right.$ is continuous (with respect to the order topology on $M$ and $M \cup A$ induced from $\bar{M}$ ) and either constant or strictly monotonic.

Proof. By Theorem 3.4 there is a partition of $\operatorname{dom}(f)$ into a finite set and finitely many definable convex sets such that on each such convex set $f$ is locally constant, locally increasing, or locally decreasing.

We now fix one such definable convex set $J \subset \operatorname{dom}(f)$ on which, say, $f$ is locally increasing; the argument is virtually the same if $f$ is locally constant or decreasing on $J$. There is a definable equivalence relation $E$ on $J$ whose classes are the maximal convex subsets of $J$ on which $f$ is strictly increasing. Since $\mathfrak{M}$ is non-valuational, this equivalence relation can have only finitely many infinite classes.

So the proposition follows if we show that if $J^{*}$ is a definable convex set for which $f\left\lceil_{J^{*}}\right.$ is strictly increasing, then there is a partition of $J^{*}$ into a finite set and finitely many definable convex sets such that the restriction of $f$ to each convex set is continuous. For this, we define an equivalence relation $E^{*}$ on $M$ by putting $u E^{*} v$ if and only if $u=v$, or $u<v$ and there is no $x \in J^{*}$ satisfying $u<f(x)<v$, 
or $v<u$ and there is no $x \in J^{*}$ satisfying $v<f(x)<u$. Clearly the $E^{*}$-classes are convex sets, and as $\mathfrak{M}$ is non-valuational there are finitely many infinite $E^{*}$-classes, say, $C_{1}, \ldots, C_{m}$, where $C_{1}<\ldots<C_{m}$. It is clear that $f$ is continuous on each of $\left\{x \in J^{*}: f(x)<C_{1}\right\},\left\{x \in J^{*}: C_{i}<f(x)<C_{i+1}\right\}$ for $i=1, \ldots, m-1$, and $\left\{x \in J^{*}: C_{m}<f(x)\right\}$.

We now define the kind of cells that appear in the improved cell decomposition theorem, and state the main results of this subsection.

Definition 6.5. A strong $n$-cell is a definable subset of $M^{n}$ which is an $n$-cell in the sense of Definition 4.5 and which also satisfies the condition that all the boundary functions (in the recursive definition of $n$-cells) are continuous.

We observe for later use that every strong cell either is open or has an injective projection onto a strong cell of lower dimension.

Theorem 6.6. Let $\mathfrak{M}$ be a non-valuational weakly o-minimal expansion of an ordered field. Then, for every $n$ and every $p \in \mathbb{N}$, if $X_{1}, \ldots, X_{p}$ are definable subsets of $M^{n}$, then there is a partition of $M^{n}$ into strong $n$-cells such that each $X_{j}$ for $j=1, \ldots, p$ is the union of cells in this partition.

Theorem 6.7. Let $\mathfrak{M}$ be a non-valuational weakly o-minimal expansion of an ordered field. If $\phi(x, \bar{y})$ is a formula in the language for $\mathfrak{M}$ with $l(\bar{y})=n$, then there is a positive integer $K$ such that for all $\bar{a} \in M^{n}$, the set $\{x \in M: \mathfrak{M} \models \phi(x, \bar{a})\}$ is the union of at most $K$ convex sets.

Theorem 6.8. Let $\mathfrak{M}$ be a non-valuational weakly o-minimal expansion of an ordered field. If $f: M^{n} \longrightarrow \bar{M}$ is an $\mathfrak{M}$-definable function, then there is a partition of $\operatorname{dom}(f)$ into strong $n$-cells $X_{1}, \ldots, X_{r}$ such that $f \Gamma_{X_{i}}$ is continuous for each $i=1, \ldots, r$, where $X_{i}$ carries its subspace topology.

Notice that Theorem 6.7 implies that the theory of a non-valuational weakly o-minimal expansion of an ordered field is weakly o-minimal. These theorems are proved by a multiple induction, as in the o-minimal case on which both the theorems and their proof are patterned (see [14]). The crucial ingredient is Lemma 6.10 below, whose proof - for purposes of exposition - we defer to the next subsection.

It is convenient at this point to introduce some notation and terminology. Let $E \subseteq M^{2} \times M^{n}$ be an $\mathcal{M}$-definable family of equivalence relations $E_{\bar{a}}=\{(u, v)$ : $(u, v, \bar{a}) \in E\}$, where $\bar{a}$ ranges over $M^{n}$, such that for every $\bar{a} \in M^{n}$ each $E_{\bar{a}}$ equivalence class is convex.

We illustrate with an important example for what follows.

Example 6.9. Let $R \subset M \times M^{n}$ be definable in $\mathcal{M}$ and let $E^{R} \subseteq M^{2} \times M^{n}$ be given by

$$
(u, v, \bar{a}) \in E^{R} \quad \Leftrightarrow \quad[u, v] \times\{\bar{a}\} \subseteq R \text { or }[u, v] \times\{\bar{a}\} \cap R=\emptyset .
$$

Returning now to the general setting, for each $\bar{a} \in M^{n}$ we set

$$
A_{\bar{a}}:=\left\{\sup C: C \text { is an } E_{\bar{a}} \text {-class }\right\},
$$

and put

$$
A_{E}:=\bigcup\left(A_{\bar{a}}: \bar{a} \in M^{n}\right) \backslash\{\infty\} .
$$


Then $A_{E}$ is a sort in $\bar{M}$, which we call the sort determined by $E$. We call the sort $A_{E}$ fiberwise finite if $A_{\bar{a}}$ is finite for all $\bar{a} \in M^{n}$ and we say that it is uniformly fiberwise finite if there is some $N \in \mathbb{N}$ such that $\left|A_{\bar{a}}\right| \leq N$ for all $\bar{a} \in M^{n}$.

We observe that if $\mathcal{M}$ is weakly o-minimal and for every $\mathcal{M}$-definable $E$ such that $A_{E}$ is fiberwise finite it is also true that $A_{E}$ is uniformly fiberwise finite, then the theory of $\mathcal{M}$ is weakly o-minimal. If $\mathcal{M}$ is a non-valuational expansion of an ordered group, then it is not difficult to prove that the converse holds. Indeed, given a definable family $E$ of equivalence relations each with convex classes such that $A_{E}$ is fiberwise finite, the fact that all cuts are non-valuational allows each class to be split uniformly into an initial and a final segment (we leave the details to the interested reader). This yields a definable family of relations on $M$, each having the same number of connected components as the number of classes in the equivalence relation from which the relation comes (constructed by selecting the initial segment of each class, for example). That $A_{E}$ is uniformly fiberwise finite now is a consequence of the weak o-minimality of the theory of $\mathcal{M}$. We do not know if the hypothesis that $\mathcal{M}$ is a non-valuational expansion of an ordered group can be relaxed.

Theorems 6.6 6.8 are proved in a simultaneous induction. We first state the crucial technical fact needed for the inductive step in the proof of Theorem6.7 Its proof is given in Section 6.3.

Lemma 6.10. Let $\mathfrak{M}$ be a non-valuational weakly o-minimal expansion of an ordered field, and let $n \in \mathbb{N}$ be given. Assume that Theorem 6.7 holds for formulas $\phi(x, \bar{y})$ in the language for $\mathfrak{M}$ with $l(\bar{y})=n$ and that $E \subseteq M^{2} \times M^{n+1}$ is an $\mathfrak{M}$-definable family of equivalence relations such that for every $(a, \bar{c}) \in M^{n+1}$ each $E_{(a, \bar{c})}$-equivalence class is convex. Suppose also that $A_{E}$ is fiberwise finite. Then there exist a sort $A$, a partition of $M^{n}$ into finitely many definable sets $X_{1}, \ldots, X_{N}$, and for each $j \leq N$ a nonnegative integer $P_{j}$ such that there is for every $\bar{c} \in X_{j}$ a finite set $\left\{b_{1}, \ldots, b_{P_{j}}\right\} \subset M \cup A$, uniformly definable in $\bar{c}$, satisfying:

i. for all $i<P_{j}$, if $b_{i}<x_{1}<x_{2}<b_{i+1}$ then $E_{\left(x_{1}, \bar{c}\right)}$ and $E_{\left(x_{2}, \bar{c}\right)}$ have the same number of equivalence classes, with the corresponding classes both open or both singletons;

ii. the same holds for $\left(-\infty, b_{1}\right)$ and $\left(b_{P_{j}}, \infty\right)$.

Assuming Lemma 6.10 we now turn to

Proof of Theorems 6.6, 6.7, and 6.8. The theorems are proved by simultaneous induction on $n$. We only sketch the main points in the proofs, as the techniques are familiar from the o-minimal context (e.g., see [14]). The $n=1$ case is immediate for Theorem 6.6, and for 6.8 it is just Proposition 6.4. The $n=0$ case of Theorem 6.7 is trivial (and note that the $n=1$ case is covered by (C) in the induction, below). The induction now takes the following form:

A. $6.6 m, 6.7 m$, and $6.8 m$ for all $m<n+1 \Longrightarrow 6.6_{n+1}$;

B. $6.6{ }_{m}$ for all $m \leq n+1$, and $6.7 m$ and $6.8 m$ for all $m<n+1 \Longrightarrow 6.8 n_{n+1}$.

C. 6.6$]_{n}$ and $6.8{ }_{n}$ for all $m \leq n+1$, and $6.7 m$ for all $m<n+1 \Longrightarrow 6.7 h_{n+1}$.

The implication in (A) is a straightforward adaptation of the proof of Theorem 4.6.

Now we turn to (B), the inductive proof of Theorem 6.8. For this, we argue roughly as in the proof of Proposition 5.3 of [14]. The only problems occur in the proof of the second assertion of Claim 5.6, since the $b_{i}$ in that proof could lie in a 
new sort. However, we can give a more refined definition of $X_{2}$. By Proposition 6.4 and Theorem $\left[6.7 \mathrm{~h}\right.$, there is a natural number $K$ such that for all $\bar{a} \in M^{n}$ there is a partition of $M$ into at most $K$ convex sets, on each of which $f\left(\bar{a}, x_{n+1}\right)$ is either constant or strictly monotonic. There are definable functions $M^{n} \longrightarrow \bar{M}$ picking out the infima and suprema of these convex sets - as images of $\bar{a}$ - and by refining the partition of $M^{n}$ we may arrange by the induction hypothesis that all these functions are continuous. We now replace the previous set $X_{2}$ by $X_{1}^{\prime}, \ldots, X_{K}^{\prime}$, where $(\bar{a}, b) \in X_{i}^{\prime}$ if $b$ is in the $i^{t h}$ convex set on which $f\left(\bar{a}, x_{n+1}\right)$ is constant or strictly monotonic. The rest of the proof goes through as in [14].

Finally, we give the argument for $(\mathrm{C})$. Let $\phi(x, \bar{y})$ with $l(\bar{y})=n+1$ be as in the hypothesis of Theorem $6.7 h_{h+1}$ and let

$$
R=\left\{\bar{b} \in M \times M^{n+1}: \mathfrak{M} \models \phi(\bar{b})\right\} .
$$

We also let $E^{R}$ be the definable family of equivalence relations obtained from $R$ as in Example 6.9 Let us now fix $i \leq n+1$. We wish to use Lemma 6.10 and for clarity we introduce some additional notation whose purpose amounts to nothing more than a permutation of coordinates. Let $E_{(a, \bar{c})}^{i}$, where $a \in M$ and $\bar{c} \in M^{n}$, denote the definable family of equivalence relations given by

$$
(u, v) \in E_{(a, \bar{c})}^{i} \Leftrightarrow\left(u, v, c_{1}, \ldots, c_{i-1}, a, c_{i}, \ldots, c_{n}\right) \in E^{R} .
$$

Applying Lemma 6.10 to $E_{(a, \bar{c})}^{i}$, there is a natural number $t_{i}$ such that for each $\bar{c} \in M^{n}$ there is a (uniformly) $\bar{c}$-definable set $\left\{b_{1}, \ldots, b_{t_{i}}\right\}$-possibly with some of the $b_{j}$ equal - such that the $b_{j}$ satisfy (i) and (ii) in the conclusion of the lemma. The mappings $\bar{c} \mapsto b_{1}, \ldots, \bar{c} \mapsto b_{t_{i}}$ are all definable. By the induction hypothesis we can partition $M^{n}$ into strong cells such that on each cell these functions all are continuous. This partition of $M^{n}$ together with these functions gives rise in the obvious way to a partition $\mathcal{P}_{i}$ of $M^{n+1}$.

Carrying out this procedure for each $i=1, \ldots, n+1$, we obtain partitions $\mathcal{P}_{1}, \ldots, \mathcal{P}_{n+1}$ of $M^{n+1}$ into finitely many sets. Let $Q \subset M^{n+1}$ consist of all $\bar{d} \in$ $M^{n+1}$ such that the least equivalence class of $E_{\bar{d}}^{R}$ (under $<$ ) is contained in $R \cap(M \times$ $\{\bar{d}\}$ ). Using the induction hypothesis that Theorem [6.6 $n$ holds for all $m \leq n+1$, we obtain a partition $\mathcal{P}$ of $M^{n+1}$, refining $\mathcal{P}_{1}, \ldots, \mathcal{P}_{n+1}$ and respecting $Q$, such that each set in $\mathcal{P}$ is an open strong cell in $M^{n+1}$ or has an injective projection onto a strong cell of lower dimension. Applying the induction hypothesis that Theorem 6.7] holds for all $m<n+1$, if $C \subseteq M^{n+1}$ is a strong cell in $\mathcal{P}$ for which the latter alternative holds, then there is a $K_{C} \in \mathbb{N}$ such that, for all $\bar{d} \in C$, the set $R \cap(M \times\{\bar{d}\})$ consists of no more than $K_{C}$ convex sets. So it remains to handle the case that $C$ is open in $M^{n+1}$. We now assert that if $\bar{y}_{1}, \bar{y}_{2} \in C$ and $\left\{w:\left(w, \bar{y}_{1}\right) \in R\right\}$ consists of $k$ convex sets, then so does $\left\{w:\left(w, \bar{y}_{2}\right) \in R\right\}$. Indeed, suppose this is false. Then for some $k \in \mathbb{N}$, the set

$$
Y_{k}:=\{\bar{y} \in C:\{w:(w, \bar{y}) \in R\} \text { consists of } k \text { convex sets }\}
$$

is a non-empty proper subset of $C$. We now invoke Theorem 6.6 for $m=n+1$ once more to partition $C$ into strong cells respecting both $Y_{k}$ and $C \backslash Y_{k}$. Since all boundary functions of cells in this partition are continuous, it follows that there is an open box $B \subset C$ with $B \cap Y_{k} \neq \emptyset$ and $B \cap\left(C \backslash Y_{k}\right) \neq \emptyset$. Observe now that none of the graphs of the functions defined in the previous paragraph (for all $i=1, \ldots, n+1)$ intersect $C$. For $i=1, \ldots, n+1$ let $\pi_{i}: M^{n+1} \longrightarrow M^{n}$ be the projection which drops the $i^{\text {th }}$ coordinate, and let us fix $i=1, \ldots, n+1$. 
The conclusion of Lemma 6.10 combined with the fact that $C \subset Q$ or $C \cap Q=\emptyset$ yields for every $\bar{c}=\left(c_{1}, \ldots, c_{n}\right) \in \pi_{i}(B)$ that the number of convex components of $R \cap(M \times\{\bar{d}\})$ is constant as we vary $a \in M$ with $\bar{d}=\left(c_{1}, \ldots, c_{i-1}, a, c_{i+1}, \ldots, c_{n}\right) \in$ $B$. Since this holds for each $i=1, \ldots, n+1$, working along each axis in turn we see that the number of convex components of $R \cap(M \times\{\bar{d}\})$ is constant for all $\bar{d} \in B$, a contradiction.

\subsection{Proof of Lemma 6.10 We begin with}

Lemma 6.11. We may suppose that for each $\bar{a} \in M^{n+1}$ and infinite $E_{\bar{a}}$-class $C$, there are $u, v \in C$ such that $1<v-u$.

Proof. By adjusting each equivalence relation $E_{\bar{a}}$ uniformly over the parameters $\bar{a}$, if necessary, we may assume for each $\bar{a}$ that there are no singleton $E_{\bar{a}}$-classes and that every infinite $E_{\bar{a}}$-class is open on the right.

For each $\bar{a} \in M^{n+1}$ let

$$
N(\bar{a}):=\left\{\delta \in M: \delta>0 \wedge\left(\forall C \in M / E_{\bar{a}}\right)\left(\exists y_{1}, y_{2} \in C\right) y_{2}-y_{1}=\delta\right\} .
$$

We now define a new equivalence relation $E_{\bar{a}}^{\prime}$ by $\left(b_{1}, b_{2}\right) \in E_{\bar{a}}^{\prime}$ if and only if $b_{1}=b_{2}$ or there is some $\delta_{0} \in N(\bar{a})$ such that $\left(\left(\delta_{0} / 2\right) b_{1},\left(\delta_{0} / 2\right) b_{2}\right) \in E_{\bar{a}}$ and also for all $\delta \in N(\bar{a})$ with $\delta>\delta_{0}$ we have $\left(\left(\delta_{0} / 2\right) b_{1},(\delta / 2) b_{1}\right) \in E_{\bar{a}}$ and $\left(\left(\delta_{0} / 2\right) b_{2},(\delta / 2) b_{2}\right) \in E_{\bar{a}}$. It is easily verified for all $\bar{a} \in M^{n+1}$ that $E_{\bar{a}}^{\prime}$ is an equivalence relation and all $E_{\bar{a}}^{\prime}$ equivalence classes are convex. Using the fact that $\mathfrak{M}$ is non-valuational, it is not difficult to prove that every infinite class has diameter greater than 1.

Let $M / E_{\bar{a}}$ denote the set of equivalence classes of $E_{\bar{a}}$ and let $b / E_{\bar{a}}$ represent the $E_{\bar{a}}$-class of $b \in M$ (and likewise for $E_{\bar{a}}^{\prime}$ ). We now define a mapping $\chi: M / E_{\bar{a}}^{\prime} \longrightarrow$ $M / E_{\bar{a}}$. Since $\mathfrak{M}$ is non-valuational, for every $b \in M$ there is a $\delta_{0} \in N(\bar{a})$ such that for all $\delta \in N(\bar{a})$ with $\delta>\delta_{0}$ we have $\left(\delta_{0} / 2\right) b E_{\bar{a}}(\delta / 2) b$. We then let

$$
\chi\left(b / E_{\bar{a}}^{\prime}\right)=\frac{\delta_{0}}{2} b / E_{\bar{a}} .
$$

It is easy to see that $\chi$ is well-defined and that $\chi$ is injective. Since $\mathfrak{M}$ is nonvaluational, it follows also that $\chi$ is surjective. Replacing $E_{\bar{a}}$ by $E_{\bar{a}}^{\prime}$, we finish the proof of the lemma.

A boundary point of $E$ is a point $(b, \bar{a}) \in\left(M \cup A_{E}\right) \times M^{n+1}$, where $b \in A_{\bar{a}}$, and we denote by $\partial E$ the set of boundary points of $E$.

Definition 6.12. Let $\bar{c} \in M^{n}$ and let $A$ be a sort containing $A_{E}$. A point $(a, d, \bar{c}) \in$ $(M \cup A) \times M \times M^{n}$ is a nasty point if one of the following holds:

(a) $(a, d, \bar{c})$ is not a boundary point of $E$, but every open box $B=J \times I \times\{\bar{c}\} \subset$ $M^{2} \times\{\bar{c}\}$ with $(a, d, \bar{c})$ in its interior contains a boundary point of $E$;

(b) $(a, d, \bar{c})$ is a boundary point of $E$, and there is no open box $B=J \times I \times\{\bar{c}\} \subset$ $M^{2} \times\{\bar{c}\}$ with $(a, d, \bar{c}) \in \operatorname{Int} B$ such that

$$
\{(u, v):(v, u, \bar{c}) \text { is a boundary point of } E \text { lying in } B\}
$$

forms the graph of a continuous function from $I$ to $M \cup A_{E}$.

For the remainder of this section, $A$ always denotes a sort containing $A_{E}$.

Lemma 6.13. Let $\bar{c} \in M^{n}$ and $d \in M$. Then there is no open interval $J \subset M \cup A$ such that each point $(b, d, \bar{c})$, where $b \in J$, is nasty. 
Proof. Suppose for a contradiction that there is such an interval $J$. Let $b \in J \cap M$ be such that $b$ lies in the interior of an infinite equivalence class of $E_{d \bar{c}}$. Choose $b_{1}, b_{2} \in J \cap M$ such that $b_{1}<b<b_{2}, b_{2}-b<1 / 2$, and $b-b_{1}<1 / 2$. By Lemma 6.11 for each $w \in M$ there is at most one boundary point of $E$ in $\left(b_{1}, b_{2}\right) \times\{w\} \times\{\bar{c}\}$. Let $(f(w), w, \bar{c})$ denote this boundary point, if it exists. So $f$ is a definable partial function from $M$ to $M \cup A_{E}$. By weak o-minimality and the assumption that $(b, d, \bar{c})$ is nasty, there is an interval $\left(e_{1}, e_{2}\right)$ with $e_{1}<d<e_{2}$ such that one of the following holds:

(i) $f(w)$ is defined for all $w \in\left(e_{1}, d\right)$ and no $w \in\left(d, e_{2}\right)$;

(ii) $f(w)$ is defined for all $w \in\left(d, e_{2}\right)$ and no $w \in\left(e_{1}, d\right)$;

(iii) $f(w)$ is defined for all $w \in\left(e_{1}, e_{2}\right) \backslash\{d\}$.

Furthermore, by Proposition 6.4 we may suppose that $f$ is monotonic or constant or undefined on $\left(e_{1}, d\right)$, and likewise on $\left(d, e_{2}\right)$. Under each of the possibilities (i)-(iii) above, it is now easy to find $b^{*} \in\left(b_{1}, b_{2}\right)$ and an open box in $B \subset M^{2}$ with $\left(b^{*}, d\right)$ in its interior such that $B \times\{\bar{c}\}$ contains no boundary points of $E$. This contradicts that $\left(b^{*}, d, \bar{c}\right)$ must be nasty.

Lemma 6.14. Let $\bar{c} \in M^{n}$. For all $d \in M$, we have that $\{b \in M \cup A:(b, d, \bar{c})$ is nasty\} is finite.

Proof. Suppose that the lemma is false. As $\mathfrak{M}$ is non-valuational, it follows from Theorem 6.3 that there exist $d \in M$ and an open interval $J \subset M \cup A$ such that $\{b \in J:(b, d, \bar{c})$ is nasty $\}$ is dense in $J$. By cutting down $J$ we may suppose that $(x, d, \bar{c}) \notin \partial E$ for all $x \in J$. Let $b \in J$, and let $B$ be an open box in $M^{2}$ with $(b, d) \in \operatorname{Int} B$. Then there is a nasty point $\left(b^{\prime}, d, \bar{c}\right) \in \operatorname{Int} B \times\{\bar{c}\}$, and by the definition of nastiness it follows that $B \times\{\bar{c}\} \cap \partial E \neq \emptyset$. Since $B$ is arbitrary, it follows that $(b, d, \bar{c})$ is nasty, and as $b$ was chosen to be an arbitrary element of $J$, Lemma 6.13 now yields a contradiction.

Lemma 6.15. Let $\bar{c} \in M^{n}$. For all but finitely many $d \in M$, there is no $b \in M \cup A$ with $(b, d, \bar{c})$ nasty.

Proof. Suppose that the lemma is false. Define a function $f: M \longrightarrow M \cup A$ by defining $f(x)$ to be the least $y \in M \cup A$ such that $(y, x, \bar{c})$ is nasty, if such $y$ exists, and leaving $f(x)$ undefined otherwise. By Lemma 6.14 $f$ is well-defined. By our supposition that the lemma is false and weak o-minimality, $\operatorname{dom}(f)$ contains an open interval $I$. We may assume also by Proposition 6.4 that $f$ is continuous on $I$. After replacing $I$ by a subinterval if necessary, one of the following holds:

(a) $(\forall x \in I)(f(x), x, \bar{c}) \in \partial E$;

(b) $(\forall x \in I)(f(x), x, \bar{c}) \notin \partial E$.

If alternative (a) holds, then by Lemma 6.11 for every $x \in I$ there is a box $B \subset M^{2}$ with $(f(x), x) \in \operatorname{Int} B$ such that $\partial E \cap(B \times\{\bar{c}\})=[$ Graph $f \cap B] \times\{\bar{c}\}$. This contradicts that each point $(f(x), x, \bar{c})$ is nasty. Hence, (b) holds.

Assuming that (b) holds, we now define functions $h_{1}, h_{2}: I \longrightarrow M \cup A_{E}$ by

$$
\begin{aligned}
& h_{1}(x):=\text { least } y \in M \cup A_{E}: f(x)<y,(y, x, \bar{c}) \in \partial E, \\
& h_{2}(x):=\text { least } y \in M \cup A_{E}: y<f(x),(y, x, \bar{c}) \in \partial E,
\end{aligned}
$$

both functions being left undefined if there is no such $y$. There are various cases to consider, all similar. By replacing $I$ by a subinterval if necessary, we may suppose that for $i=1,2$ either $\operatorname{dom}\left(h_{i}\right)=I$ and $h_{i}$ is continuous on $I$, or $\operatorname{dom}\left(h_{i}\right)=\emptyset$. 
We show how to handle the case for which $\operatorname{dom}\left(h_{1}\right)=\operatorname{dom}\left(h_{2}\right)=I$. Let $x \in I$. By continuity, there is a box $B=J^{\prime} \times I^{\prime} \in M^{2}$ with $(f(x), x) \in \operatorname{Int} B$, such that $\left(h_{1}(w), w\right),\left(h_{2}(w), w\right) \notin B$ for all $w \in I^{\prime}$. This contradicts that $(f(x), x)$ is nasty.

Definition 6.16. Let $\bar{c} \in M^{n}$. The point $x \in M$ is called unbounded if for all open intervals $I$ containing $x$ and all $K \in \mathbb{N}$ there is an element $w \in I$ such that $E_{w, \bar{c}}$ has at least $K$ equivalence classes.

Note the dependence on $\bar{c}$ in the definition above. This dependence will always be clear from the context in what follows.

Before continuing we need some additional notation. Let $\bar{c} \in M^{n}$. For $x \in M$ and $i \in \mathbb{N}$, define $g_{i}^{\bar{c}}(x)$ to be the $i^{t h}$ greatest element $y$ such that $(y, x, \bar{c}) \in \partial E$, if such a $y$ exists, and let $g_{i}^{\bar{c}}(x)$ be undefined otherwise. Likewise, let $g_{\ell}^{\bar{c}}(x)$ be the least element $y$ such that $(y, x, \bar{c}) \in \partial E$, if such a $y$ exists. We also define an equivalence relation $\sim_{\bar{c}}$ on $M$ by putting $u \sim_{\bar{c}} v$ if there is some $C \in M, C>0$, such that $g_{0}^{\bar{c}}(x)<C$ and $g_{\ell}^{\bar{c}}(x)>-C$ for all $x \in[u, v]$, or if $g_{0}^{\bar{c}}$ (and hence $g_{\ell}^{\bar{c}}$ ) is undefined throughout $[u, v]$. Observe that $g_{\ell}^{\bar{c}}$, each $g_{i}^{\bar{c}}$ and $\sim_{\bar{c}}$ are $\bar{c}$-definable in $\mathfrak{M}$, and uniformly so as $\bar{c}$ varies. Also note that each $\sim_{\bar{c}}$-class is convex.

Lemma 6.17. Let $\bar{c} \in M^{n}$ and let $\sim_{\bar{c}}$ be as above. Then there are finitely many $\sim_{\bar{c}}$-classes.

Proof. Suppose that the lemma is false. Then, as $\mathfrak{M}$ is non-valuational and all $\sim_{\bar{c}^{-}}$-classes are convex, by Theorem 6.3 there is an interval $I$ such that for all $x \in I$,

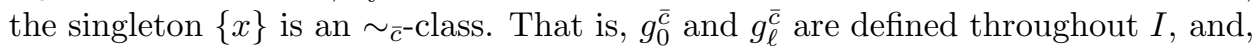
for all $x, y \in I$ with $x<y$, either $g_{0}^{\bar{c}}$ is not bounded above on $(x, y)$, or $g_{\ell}^{\bar{c}}$ is not bounded below on $(x, y)$. Since by Proposition 6.4 there is an interval $J \subset I$ on which $g_{0}^{\bar{c}}$ and $g_{\ell}^{\bar{c}}$ are constant or strictly monotonic, this is impossible.

Lemma 6.18. There is a sort $A^{*}$ containing $A_{E}$ such that for all $\bar{c} \in M^{n}$, if $x$ is any unbounded point in an open $\sim_{\bar{c}^{-}}$equivalence class, then there is some $y \in M \cup A^{*}$ such that $(y, x, \bar{c})$ is nasty.

Proof. We first prove a weaker result. Let $\bar{c} \in M^{n}$, and let $I$ be an open convex $\sim_{\bar{c}^{-}}$-class. We show that if $x \in I$ is unbounded, then there is a sort $A$ containing $A_{E}$ and some $y \in M \cup A$ such that $(y, x, \bar{c})$ is nasty. After we have established this, we indicate how to obtain the stronger conclusion of the lemma.

For a contradiction we suppose that $\bar{c} \in M^{n}$, that $I$ is an open convex $\sim_{\bar{c}^{-}}$ class, and that for some unbounded $x \in I$ there is no sort $A$ (depending on $\bar{c}$ and $x$ ) containing $A_{E}$ for which there is some $y \in M \cup A$ such that $(y, x, \bar{c})$ is nasty. Since $x$ is unbounded and $I$ is a single $\sim_{\bar{c}^{-}}$class, we see that $g_{0}^{\bar{c}}$ and $g_{\ell}^{\bar{c}}$ are defined throughout $I$ and bounded in an open neighborhood of $x$. Without loss of generality, since $x$ is unbounded we may assume for every $i \in \mathbb{N}$ that there is some $d_{i} \in M$, with $d_{i}<x$, such that throughout $\left(d_{i}, x\right)$ the function $g_{i}^{\bar{c}}$ is defined, continuous, and either constant or strictly monotonic (the other alternative - that there are $e_{i} \in M$ with $x<e_{i}$ such that throughout $\left(x, e_{i}\right)$ the function $g_{i}^{\bar{c}}$ is defined, continuous, and either constant or strictly monotonic - is handled similarly). For each $i \in \mathbb{N}$, define

$$
Y_{i}:= \begin{cases}\sup \left\{g_{i}^{\bar{c}}(z): d_{i}<z<x\right\} & \text { if } g_{i}^{\bar{c}} \text { is not monotonically decreasing on }\left(d_{i}, x\right), \\ \inf \left\{g_{i}^{\bar{c}}(z): d_{i}<z<x\right\} & \text { otherwise. }\end{cases}
$$

We now put $A:=A_{E} \cup\left\{Y_{i}: i \in \mathbb{N}\right\}$. 
To see that $A$ is a sort, we rely on Lemma 6.11, which tells us that for each $\bar{a}=(x, \bar{c}) \in M^{n+1}$ the distance between any two boundary points of $E_{\bar{a}}$-classes is at least 1 . Hence we can uniformly define the points $Y_{i}$ by working inside open boxes of the form $B=I \times J \times\{\bar{c}\}$, where $|I| \leq 1 / 2$ and $x \in J$. We leave details of this to the reader. For each $i$, since $\left(Y_{i}, x, \bar{c}\right)$ is not nasty it must be a boundary point of $E$, so for each $i \in \mathbb{N}$ there is $j \in \mathbb{N}$ such that $Y_{i}=g_{j}^{\bar{c}}(x)$. From this, it follows that $E_{x, \bar{c}}$ does not have finitely many classes, contradicting the hypothesis that $A_{E}$ is fiberwise finite.

Now we show how to obtain the full strength of the lemma, i.e., that there is a fixed sort $A^{*}$ such that for all $\bar{c} \in M^{n}$, if $x$ is any unbounded point in a $\sim_{\bar{c}^{-}}$equivalence class, then there is some $y \in M \cup A^{*}$ such that $(y, x, \bar{c})$ is nasty. First observe from the proof above that all potential nasty points have the form $Y_{i}=\sup \left\{g_{i}^{\bar{c}}(z): d_{i}<z<x\right\}$ or $\inf \left\{g_{i}^{\bar{c}}(z): d_{i}<z<x\right\}$. To verify that we can define a sort containing all such points, we rely on Lemma 6.11 just as we did in the preceding paragraph to see that $A$ defined there is a sort. As we also did there, we can uniformly define such points $Y_{i}$ by working inside open boxes of the form $B=I \times J \times\{\bar{c}\}$ where $|I| \leq 1 / 2$ and $x \in J$. Again, we omit the details.

Let $\bar{c} \in M^{n}$. We call a convex set $J$ good for $\bar{c}$ if $\partial E \cap(\bar{M} \times J \times\{\bar{c}\})=\emptyset$ or there is some $n \in \mathbb{N}$ such that $g_{0}^{\bar{c}}, \ldots, g_{n}^{\bar{c}}$ are defined and continuous throughout $J$ and $g_{n+1}^{\bar{c}}$ is nowhere defined on $J$.

Lemma 6.19. Let $A^{*}$ be as in the conclusion of Lemma 6.18. For each $\bar{c} \in M^{n}$ define an equivalence relation $F_{\bar{c}}$ on $M$ by putting $u F_{\bar{c}} v$ if either $u=v$ or $u \sim_{\bar{c}} v$, there are no $x \in[u, v]$ and $y \in M \cup A^{*}$ such that $(y, x, \bar{c})$ is nasty, and some good convex set contains both $u$ and $v$. Then the equivalence relation $F_{\bar{c}}$ is uniformly $\bar{c}$-definable, and there are finitely many $F_{\bar{c}}$-equivalence classes, all of which are convex.

Proof. It is clear from the definition of $F_{\bar{c}}$ that all $F_{\bar{c}}$-classes are convex. Assuming that $F_{\bar{c}}$ is definable, from the assumption that $\mathfrak{M}$ is of non-valuational type it follows that there are just finitely many $F_{\bar{c}}$-classes.

Thus it remains to prove that $F_{\bar{c}}$ is (uniformly) $\bar{c}$-definable. By Lemma 6.17 and Lemma 6.15 we can assume that $M$ is partitioned (uniformly) $\bar{c}$-definably into

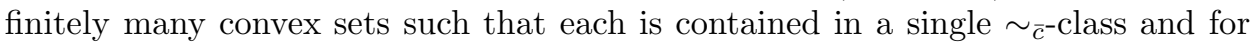
no $x$ in one of these sets is there a $y \in M \cup A^{*}$ with $(y, x, \bar{c})$ a nasty point. Observe that if $I$ is such an infinite convex set, then by Lemma 6.18 there are no unbounded points of $I$.

For $e, f \in I$ with $e<f$, where $I$ is an infinite convex set as above, put $e \bowtie_{\bar{c}} f$ if the following does not occur: there are $a, b \in I$ with $e \leq a<b \leq f$ and an interval $[u, v]$ in $M$ of length less than 1 such that $\partial E \cap([u, v] \times[a, b] \times\{\bar{c}\})$ is the graph of a continuous, and constant or monotonic, function $h$ whose domain is a non-empty proper convex subset of $[a, b]$, and there are $c, d \in M$ with $u<c<d<v$ such that for all $x \in[a, b]$, if $h(x)$ is defined then $c<h(x)<d$. The idea behind this condition is roughly that the graph of $h$ should have an 'endpoint' in the box $(u, v) \times[a, b]$.

It is easy to see that if $x F_{\bar{c}} y$ then $x \bowtie_{\bar{c}} y$. Since $\bowtie_{\bar{c}}$ is (uniformly) $\bar{c}$-definable, to prove the lemma it suffices to show that if $\neg x F_{\bar{c}} y$ then $x \rtimes_{\bar{c}} y$. To this end, let $C \subset I$ be an $F_{\bar{c}}$-class, and suppose there is some $x \in I$ with $x<C$. We consider the case that $C$ is not a singleton and $\alpha:=\inf C \notin C$; these possibilities are easily 
handled using Proposition 6.4 and the fact that $I$ does not contain any unbounded points $y \in C$. We must show that $x \bowtie_{\bar{c}} y$. There is some $n \in \mathbb{N}$ such that $g_{0}, \ldots, g_{n}$ are defined, continuous, and constant or monotonic on $(\alpha, y)$, but $g_{n+1}$ is undefined on $(\alpha, y)$. We also may suppose that there is some $d<\alpha$ such that $g_{0}, \ldots, g_{n}$ are defined and continuous on $(d, y)$, as it is easy to see that $x \downarrow_{\bar{c}} y$ if this does not hold. Furthermore, there must be some $b<\alpha$ such that $g_{n+1}$ is defined throughout $(b, \alpha)$. For if not, then by weak o-minimality there would be some $b<\alpha$ such that $g_{n+1}$ is undefined throughout $(b, \alpha)$. In this case we would have either that $\alpha \in M$ with $g_{n+1}(\alpha)$ defined and hence $x \downarrow_{\bar{c}} y$, as required, or that $z F_{\bar{c}} y$ for $z \in(b, \alpha)$, contrary to hypothesis. We may suppose that $g_{n+1}$ is continuous, and constant or strictly monotonic on $(b, \alpha)$. We suppose also that it is strictly increasing, the other cases being similar. Let $c:=\sup \left\{g_{n+1}(w): w \in(b, \alpha)\right\}$. Then by Lemma 6.11 we have $c \neq \lim _{z \rightarrow \alpha^{-}} g_{n}(z)$. It now follows easily that $x \not \aleph_{\bar{c}} y$, as required.

Conclusion of the proof of Lemma 6.10. By Lemma 6.19] and the hypothesis for Lemma 6.10 that Theorem 6.7 holds for formulas with $n$ parameter variables, we see that there is some $K \in \mathbb{N}$ such that for every $\bar{c} \in M^{n}$ the number of $F_{\bar{c}}$-classes is no greater than $K$. Thus, there exist a sort $A$-namely $A:=\bigcup_{\bar{c} \in M^{n}} A_{F_{\bar{c}}}$ and a partition of $M^{n}$ into finitely many definable sets $X_{1}, \ldots, X_{N}$ such that for each $j \leq N$ there some $P_{j} \leq K$ for which for all $\bar{c} \in X_{j}$ there is a finite set $\left\{b_{1}, \ldots, b_{P_{j}}\right\} \subset M \cup A$ uniformly definable in $\bar{c}$-as the suprema and infima of the $F_{\bar{c}}$-classes - satisfying the conclusion of the lemma. This completes the proof.

\section{FURTHER OBSERVATIONS AND QUESTIONS}

7.1. The exchange property for algebraic closure. We first characterize those weakly o-minimal theories for which algebraic closure has the exchange property. Before stating the result we introduce some terminology.

Definition 7.1. Let $\mathfrak{M}$ be an expansion of an ordered field. A bad function for $\mathfrak{M}$ is a definable injective function having the property that there is a definable non-trivial convex valuation $v: M \longrightarrow \Gamma$ with valuation ring $R$ such that for every $x \in \operatorname{dom}(f)$ the value $f(x)$ is a coset in $(M,+)$ of a non-trivial convex ideal of $R$, and $f(x) \cap f(y)=\emptyset$ for all distinct $x, y \in \operatorname{dom}(f)$.

Theorem 7.2. Let $\mathfrak{M}$ be an $\omega$-saturated weakly o-minimal structure.

(a) The following are equivalent.

(i) Algebraic closure in $\mathfrak{M}$ does not have the exchange property.

(ii) There is a locally constant definable partial function $f: M \longrightarrow M$ for which there is no partition of $\operatorname{dom}(f)$ into finitely many definable convex sets such that the restriction of $f$ to each is constant.

(iii) There is a strictly monotonic definable partial function $f$ from an interval $I \subseteq M$ to a sort in $\bar{M}$ for which there is no partition of $\operatorname{dom}(f)$ into finitely many definable convex sets such that the restriction of $f$ to each is continuous.

(b) If in addition $\mathfrak{M}$ is an expansion of an ordered field, then the above conditions also are equivalent to

(iv) $\mathfrak{M}$ has a bad function.

Proof. We begin with (i) $\Rightarrow$ (ii). Suppose that algebraic closure in $\mathfrak{M}$ does not have the exchange property. Then there are $\bar{a}, b, c \in M$ with $c \in \operatorname{acl}(b \bar{a}) \backslash \operatorname{acl}(\bar{a})$ 
and $b \notin \operatorname{acl}(\bar{a} c)$. Since $M$ is totally ordered, algebraic closure is equal to definable closure, and so there is a $\bar{a}$-definable partial function $f$ with $f(b)=c$. As $b \notin \operatorname{acl}(\bar{a} c)$, we see that $\{x: f(x)=c\}$ is infinite and so by weak o-minimality contains an interval. Hence, since $c \notin \operatorname{acl}(\bar{a})$, there is an infinite definable set $I$ such that $\{x \in M: f(x)=y\}$ is infinite for all $y \in I$. For each $y \in I$ we have that $D_{y}=\{x \in M: f(x)=y\}$ is a finite union of convex sets, and so we clearly may suppose that each $D_{y}$ consists of a single convex set. By restricting the domain of $f$ so that $\operatorname{ran}(f)=I$ we now obtain a locally constant function as in (ii).

Now we turn to (ii) $\Rightarrow$ (iii). Let $f: M \longrightarrow M$ be a locally constant definable partial function as in (ii). Let

$$
A:=\{\sup B: B \text { is a maximal convex set on which } f \text { is constant }\} \text {. }
$$

Then $A$ is a sort in $\bar{M}$.

By weak o-minimality, for each $z \in \operatorname{ran}(f)$ there are only finitely many maximal convex sets on which $f$ takes on the value $z$. By cutting down $\operatorname{dom}(f)$ if necessary we may assume that for each $z \in \operatorname{ran}(f)$ there is a unique maximal convex $B_{z} \subseteq \operatorname{dom}(f)$ such that $f(x)=z$ for all $x \in B_{z}$. It follows from (ii) and weak o-minimality that $\operatorname{ran}(f)$ contains an infinite interval $J$. Now define $g: J \longrightarrow A$ by $g(z):=\sup B_{z}$. Certainly $g$ is injective. By Theorem 3.4 there is an interval $I \subseteq J$ on which $g$ is monotonic. Since $f$ is locally constant, it follows immediately that $g$ is nowhere continuous.

For the proof of (iii) $\Rightarrow$ (i), let $f: M \longrightarrow A$ be a strictly monotonic $\bar{a}$-definable function which is not (finitely) piecewise continuous. We may suppose that for each $x \in \operatorname{dom}(f)$ there is some $y \in M$ such that $y>f(x)$ and $\operatorname{ran}(f) \cap(f(x), y)_{\bar{M}}=\emptyset$. Choose such an $x$ and $y$, with $x \notin \operatorname{acl}(\bar{a})$ - by $\omega$-saturation-and pick $z \in(f(x), y)$ with $z \notin \operatorname{acl}(\bar{a} x)$. But then $x \in \operatorname{acl}(\bar{a} z)$, so algebraic closure does not have the exchange property.

Now we prove the additional equivalence given in part (b). We first show that (iv) $\Rightarrow$ (i). Suppose that $\mathfrak{M}$ has an $\bar{a}$-definable bad function $f$ whose domain is a convex set $I$, and let $v: M \longrightarrow \Gamma$ be the corresponding valuation. By $\omega$-saturation there is some $c \in \operatorname{dom}(f) \backslash \operatorname{acl}(\bar{a})$. Since $f(c)$ is a coset of an infinite ideal and hence an infinite definable subset of $M$, there is some $b \in M$ such that $b \in f(c)$ and $b \notin \operatorname{acl}(\bar{a} c)$. We also have that $c \in \operatorname{acl}(\bar{a} b)$, as $c^{\prime} \neq c$ implies that $b \notin f\left(c^{\prime}\right)$. Since $c \notin \operatorname{acl}(\bar{a})$, it follows that algebraic closure in $\mathfrak{M}$ does not have the exchange property.

To complete the proof of the theorem we establish that (iii) $\Rightarrow$ (iv). Let $f$ : $M \longrightarrow A$ be a strictly monotonic definable partial function as in (iii). For each $x \in \operatorname{dom}(f)$ let $g_{1}(x) \in \bar{M}$ be chosen least such that

$$
\operatorname{ran}(f) \cap\left(g_{1}(x), f(x)\right)=\emptyset,
$$

and let $g_{2}(x) \in \bar{M}$ be chosen greatest such that

$$
\operatorname{ran}(f) \cap\left(f(x), g_{2}(x)\right)=\emptyset .
$$

Then

$$
\left\{g_{1}(x): x \in \operatorname{dom}(f)\right\} \cup\left\{g_{2}(x): x \in \operatorname{dom}(f)\right\}
$$

is a sort in $\bar{M}$. Either there are infinitely many $x \in \operatorname{dom}(f)$ such that $g_{1}(x)<f(x)$, or there are infinitely many $x \in \operatorname{dom}(f)$ such that $f(x)<g_{2}(x)$. Without loss of generality we suppose the former and, after restricting $f$ if necessary, assume that for all $x \in \operatorname{dom}(f)$, we have $g_{1}(x)<f(x)$. For each $x \in \operatorname{dom}(f)$ let $A_{x}:=$ 
$\left(g_{1}(x), f(x)\right)$. By restricting $f$ and using the fact that $\mathfrak{M}$ is a field, we additionally may suppose that $|f(x)|<1$ for all $x \in \operatorname{dom}(f)$. It follows that $g_{1}(x) \leq-1$ for at most one value of $x$, and so by restricting $\operatorname{dom}(f)$ further we can assume that $A_{x} \subseteq(-1,1)$ for all $x \in \operatorname{dom}(f)$. As the sets $A_{x}$ are uniformly definable, we can apply the proof of implication (iii) $\Rightarrow$ (ii) of Theorem 6.3 and the proof of Lemma 6.2 Thus, by restricting $f$ again if necessary, we may suppose for all $x \in \operatorname{dom}(f)$ that the set $I_{x}:=\left\{a_{1}-a_{2}: a_{1}, a_{2} \in A_{x}\right\}$ is a proper non-empty subgroup of $(M,+)$ which gives rise to a definable convex valuation ring $R_{x}$. The proof of Lemma 6.2 further yields that each $I_{x}$ is an ideal in $R_{x}$. Hence, each set $A_{x}$ is a coset of $I_{x}$, and for $x \neq y$ in $\operatorname{dom}(f)$ we have that $A_{x} \cap A_{y}=\emptyset$.

We complete the proof by showing how to obtain a single valuation ring $R$ such that for all $x \in \operatorname{dom}(f)$ the sets $A_{x}$ are cosets of ideals in this valuation ring, and hence that the mapping $x \mapsto A_{x}$ is a bad function. As the valuation rings $R_{x}$ are convex, they are ordered by inclusion. Also, every proper convex subring of $\mathfrak{M}$ containing 1 is a convex subring of its field of fractions, $\mathfrak{M}$, and so is a convex valuation ring in $\mathfrak{M}$ (see [5]). Since for each $x \in \operatorname{dom}(f)$ the ring $R_{x}$ contains the (convex) ring of finite elements in $\mathfrak{M}$, we thus see that $R=\bigcap_{x \in \operatorname{dom}(f)} R_{x}$ is a non-empty convex valuation ring in $\mathfrak{M}$. As $I_{x} \subseteq(-1,1) \subseteq R$ and $R \subseteq R_{x}$ for all $x \in \operatorname{dom}(f)$, we also have for all $x \in \operatorname{dom}(f)$ that $I_{x}$ is a non-trivial ideal of $R$. Hence the mapping $x \mapsto A_{x}$ for $x \in \operatorname{dom}(f)$ is a bad function, as required.

7.2. The independence property. We next show that Corollary 3.10 of [22] has a straightforward generalisation. This gives an alternative proof of the result of Mathews [20] that real closed rings do not have the independence property. Our result takes on importance as a consequence of the results in [17]. There it is proved that for every formula $\varphi(\bar{x}, \bar{y})$ in the language of a structure $\mathfrak{M}$, the uniformly definable family

$$
\left\{\{\bar{a}: \mathfrak{M} \models \varphi(\bar{a}, \bar{b})\}: \bar{b} \in M^{k}\right\},
$$

where the length of $\bar{y}$ equals $k$, is a Vapnik-Chervonenkis class if and only if $\varphi(\bar{x}, \bar{y})$ does not have the independence property with respect to $\mathfrak{M}$.

Proposition 7.3. If $T$ is a weakly o-minimal theory, then $T$ does not have the independence property.

Proof. Let $\mathfrak{M}$ and $\mathfrak{N}$ be models of $T$ with $\mathfrak{M} \preceq \mathfrak{N}$, and let $p(x)$ be a 1-type over $M$. By a theorem of Poizat - see Chapter 9 of [21] - it suffices to show that $p$ has less than $2^{|M|}$ coheirs over $\mathfrak{N}$. We show that in fact $p$ has at most 2 such coheirs.

Let $C(x)$ be the cut over $M$ determined by $p$, that is, the partial type contained in $p$ of the form

$$
\left\{c \leq x: x \in C_{1}\right\} \cup\left\{x \leq c: c \in C_{2}\right\},
$$

where $M=C_{1} \cup C_{2}$ is a partition with $u<v$ for all $u \in C_{1}$ and $v \in C_{2}$. Let $A$ be the set of elements of $N$ satisfying $C(x)$.

We suppose first that $A$ is non-empty. Let $q(x)$ be a coheir of $p$. Then either

$$
\{x<a: a \in A\} \subseteq q(x),
$$

or

$$
\{a<x: a \in A\} \subseteq q(x) .
$$

We prove in either case that there is at most one possibility for $q$. Suppose that the first possibility holds; the argument in the second case is similar. For each formula 
$\phi(x)$ over $N$, we have that $\phi \in q$ if and only if $\{x: N \models \phi(x)\}$ contains a non-empty final segment of $M \cap C_{1}$. Hence $q$ is determined by $C$.

Now suppose that $A$ is empty. In this case, if there is no formula $\phi(x)$ over $N$ such that $\{a \in M: N \models \phi(a)\}=C_{1} \cap M$, then $p$ has a unique extension over $\mathfrak{N}$, and this is a coheir. Suppose, however, that there is such a formula $\phi(x)$. Then clearly $p$ has at most one coheir containing $\phi$ and at most one coheir omitting $\phi$. Thus, in all cases $p$ has at most two coheirs, as required.

7.3. Open problems. We now list some open problems. Their focus is on weakly o-minimal expansions of ordered groups and fields, where much of the impetus for the study of weakly o-minimal structures lies.

Problem 1. If $\mathfrak{M}$ is a weakly o-minimal expansion of an ordered group, or field, must it have weakly o-minimal theory?

Problem 2. Find a direct proof that every weakly o-minimal ordered field is real closed.

Problem 3. Investigate differentiability and analyticity properties of definable functions for weakly o-minimal expansions of ordered fields.

Problem 4. Is there a weakly o-minimal expansion of an ordered field in which algebraic closure does not have the exchange property?

\section{REFERENCES}

[1] T. M. Apostol, Mathematical analysis, a modern approach to advanced calculus, Reading, Massachusetts: Addison-Wesley 1957. MR 19:398c

[2] R. Arefiev, On monotonicity for weakly o-minimal structures, preprint.

[3] Y. Baisalov and B. Poizat, Paires de structures o-minimales, J. Symbolic Logic, 63 (1998) 570-578. MR 89m:03063

[4] P.J. Cameron, Some treelike objects, Quart. J. Math. (Oxford) (2), 38 (1987) 155-183. MR 89a:05009

[5] G. Cherlin, M.A. Dickmann, Real closed rings II: model theory, Ann. Pure Appl. Logic 25 (1983) 213-231. MR 86j:03033

[6] M.A. Dickmann, Elimination of quantifiers for ordered valuation rings, J. Symbolic Logic, 52 (1987) 116-128. MR 88b:03045

[7] L. van den Dries, Remarks on Tarski's problem concerning $(R,+, .$, exp $)$, Logic Colloquium '82 (G. Lolli, G. Longo, A. Marcja, eds.), Amsterdam: North-Holland 1984, 97-121. MR 86g:03052

[8] L. van den Dries, T-convexity and tame extensions II, J. Symbolic Logic 62 (1997) 14-34; 63 (1998), 1597. MR 98h:03048: CMP 99:06

[9] L. van den Dries, Tame topology and o-minimal structures, London Mathematical Society Lecture Note Series, vol. 248, Cambridge: Cambridge University Press 1998. MR 99j:03001

[10] L. van den Dries and A. Lewenberg, T-convexity and tame extensions, J. Symbolic Logic 60 (1995) 74-102. MR 96a:03048

[11] A. Macintyre, K. McKenna, L. van den Dries, Elimination of quantifiers in algebraic structures, Advances in Mathematics 47 (1983) 74-87. MR 84f:03028

[12] D. Haskell, H. D. Macpherson, 'Cell decompositions of $C$-minimal structures', Ann. Pure Appl. Logic, 66 (1994) 113-162. MR 95d:03059

[13] B. Herwig, H. D. Macpherson, G. Martin, A. Nurtazin, and J. K. Truss, On $\aleph_{0}$-categorical weakly o-minimal theories, preprint.

[14] J. Knight, A. Pillay, C. Steinhorn, Definable sets in ordered structures II, Trans. Amer. Math. Soc. 295 (1986) 593-605. MR 88b:03050b

[15] F-V. Kuhlmann, Abelian groups with contractions I, Contemporary Math. 171 (1994) 217241. MR 95:03079 
[16] F-V. Kuhlmann, Abelian groups with contractions II: weak o-minimality, in Abelian groups and modules (Proceedings of the 1994 Padova Conference; A. Facchini and C. Menini, eds.), Dordrecht: Kluwer Academic Publishers 1995, pp. 323-342. MR 97g:03044

[17] C. Laskowski, Vapnik-Chervonenkis classes of definable sets, Journal of the London Math. Society, 60 (1992) 377-384. MR 93d:03039

[18] S. MacLane, The universality of formal power series fields, Bull. Amer. Math. Soc., 45 (1939) 888-890. MR 1:102c

[19] H. D. Macpherson and C. Steinhorn, On variants of o-minimality, Annals of Pure and Applied Logic, 79 (1996) 165-209. MR 97e:03050

[20] L. Mathews, D. Phil dissertation, University of Oxford 1992.

[21] A. Pillay, An introduction to stability theory, Oxford: Oxford University Press 1983. MR 85i:03104

[22] A. Pillay, C. Steinhorn, Definable sets in ordered structures I, Trans. Amer. Math. Soc. 295 (1986) 565-592. MR 88b:03050a

[23] A. Prestel, Lectures on Formally Real Fields, Lecture Notes in Mathematics vol. 1093, Berlin: Springer-Verlag 1984. MR 86h:12013

[24] P. Ribenboim, Théorie des valuations, Montréal: Les Presses de l'Université de Montréal 1964. MR 40:2670

Department of Pure Mathematics, University of Leeds, Leeds LS2 9JT, U.K.

E-mail address: pmthdm@amsta.leeds.ac.uk

Department of Mathematics, Statistics and Computer Science, University of Illinois at Chicago, Chicago, Illinois 60607

E-mail address: marker@math.uic.edu

Department of Mathematics, Vassar College, Poughreepsie, New York 12604

E-mail address: steinhorn@vassar.edu 